Check for updates

Cite this: Mater. Adv., 2021, 2,2483

Received 26th December 2020 Accepted 11th March 2021

DOI: $10.1039 / \mathrm{d} 0 \mathrm{ma} 01016 \mathrm{~b}$

rsc.li/materials-advances

\section{The development, application, and performance of black phosphorus in energy storage and conversion}

\author{
Peng Li, (D) ab Jianguo Lu, (D) *c Hongzhi Cui, ${ }^{\star d}$ Shuangchen Ruan ${ }^{a}$ and \\ Yu-Jia Zeng (D)*ab
}

\begin{abstract}
Black phosphorus with a long history of $\sim 100$ years has recently attracted extraordinary attention and has become a promising candidate for energy storage and conversion owing to its unique layered structure, impressive carrier mobility, remarkable in-plane anisotropic properties, and tunable bandgap from $0.3 \mathrm{eV}$ in the bulk to $2.0 \mathrm{eV}$ in the monolayer. Aiming to fully dredge its potential utilization, several investigations are being carried out, from its intrinsic properties to energy-driven charge transfer behavior. This review specifically highlights the very recent progress in the synthesis and applications of black phosphorus in the energy process, including secondary battery system, supercapacitor device, and catalysis reaction. The present critical issues, challenges, and perspectives in terms of well-harnessed scalability, quality, and stability are comprehensively covered. An in-depth understanding of these aspects is of great importance for the design of black phosphorus as a multifunctional candidate in future energy storage and conversion.
\end{abstract}

\section{Introduction}

Although it was already obtained in 1914 under a high pressure of $1.2 \times 10^{9} \mathrm{~Pa}$ at $200{ }^{\circ} \mathrm{C}$ using white phosphorus as the precursor, trivial attention has been paid in the study of black phosphorus (BP) during the past century due to its stringent synthetic conditions and dim prospects for application. Then, in 1989, Morita's group improved the synthetic safety by selecting red phosphorus (RP) as the P source, considering that white phosphorus is highly flammable and toxic. ${ }^{1}$ In 2007 , Sohn and his co-workers successfully obtained BP by means of high energy mechanical milling for $54 \mathrm{~h}$ in an argon atmosphere. $^{2}$ Nevertheless, the low crystallinity of collected BP dramatically hindered its further utilization. In the same year, Nilges et al. proposed a mild mineral additive-assisted method, in which with the assistance of gold, tin, and tin iodide, BP could be produced under low pressure. ${ }^{3}$ Then, Zhao et al.

\footnotetext{
${ }^{a}$ College of Physics and Optoelectronic Engineering, Shenzhen University, Shenzhen, 518060, China. E-mail: yjzeng@szu.edu.cn

${ }^{b}$ Solid State Physics and Magnetism Section, KU Leuven, Celestijnenlaan 200 D, BE-3001 Leuven, Belgium

${ }^{c}$ State Key Laboratory of Silicon Materials, School of Materials Science and Engineering, Zhejiang University, Hangzhou 310027, China.

E-mail: lujianguo@zju.edu.cn

${ }^{d}$ Key Laboratory for Resilient Infrastructures of Coastal Cities (MOE), College of Civil and Transportation Engineering, Shenzhen University, Shenzhen 518060, P. R. China. E-mail: h.z.cui@szu.edu.cn
}

remarkably optimized the mineral additive-assisted method, realizing its implementation at atmospheric pressure with the expensive $\mathrm{Au}$ eliminated. ${ }^{4}$ These efforts prompted the subsequent exploration of BP materials in energy storage and conversion applications.

During recent years, research on black phosphorus has become a hot spot owing to its unique physicochemical properties. To further trigger its potential application or delicate hybridization in energy-related devices, two-dimensional (2D) BP with few layers or monolayer should be realized since it possesses a series of advantages including reliable strength, desirable and tunable catalytic as well as electrochemical properties. Graphene is the most representative $2 \mathrm{D}$ material and raised a wave of attention since its first identification by Geim et al. in $2004 .^{12,13}$ Although the high thermal conductivity, carrier mobility, and chemical stability promoted its systematically exploration and industrial production, the inherent drawback of zero band gap significantly hinder its application in some areas, such as catalysis. $^{14,15}$ To conquer this bottleneck, transition metal dichalcogenides (TMDs) were further explored, demonstrating impressive applications in energy storage and conversion, such as battery electrodes and catalysts over the past few years. ${ }^{16}$ Nevertheless, TMDs, taking $\mathrm{MoS}_{2}$ as an example (Fig. 1a), generally show unsatisfactory photocatalytic efficiency as a result of limited light adsorption due to the large bandgap $(\sim 2.0 \mathrm{eV})$, which is a similar situation encountered by other $2 \mathrm{D}$ semiconductors such as carbon nitride with a bandgap of $2.7 \mathrm{eV} .^{17,18}$ 
a

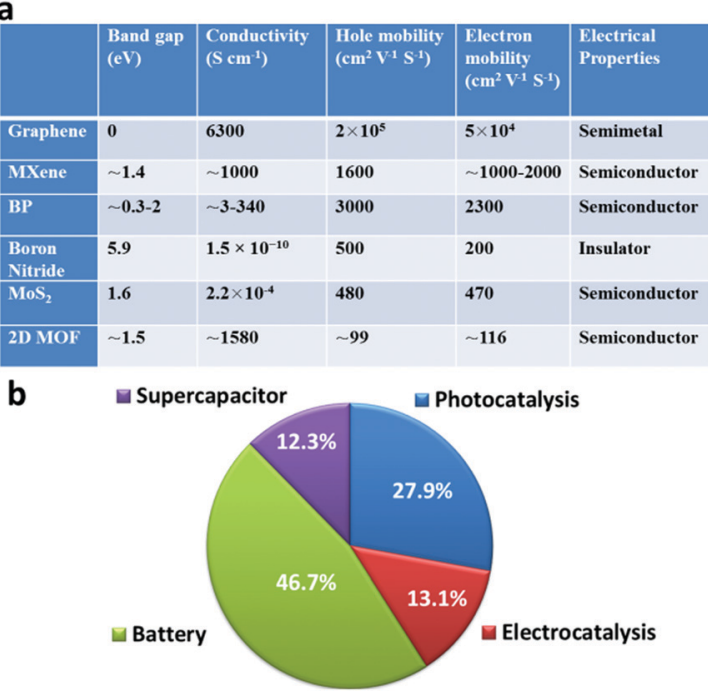

Fig. 1 (a) Comparison of common 2D materials. ${ }^{5-11}$ (b) The pie chart showing different ratios of publications on energy storage and conversion in the year of 2020 based on the data source of Web of Science.

BP with a wide thickness-dependent bandgap from 0.3-2.0 eV possesses a wide photon absorption window, implying great potential for the energy conversion process. ${ }^{19,20}$ In addition, BP shows high hole mobility $\left(1000 \mathrm{~cm}^{2} \mathrm{~V}^{-1} \mathrm{~s}^{-1}\right)$ and is active for battery devices, forming a stable alloy with a high theoretical capacity (e.g., $\left.\mathrm{Li}_{3} \mathrm{P}, 2596 \mathrm{~mA} \mathrm{~h} \mathrm{~g}{ }^{-1}\right)$. In 2014, Cui's group prepared $\mathrm{BP}$ nanoparticles with graphite (BP-G) by high-energy ball milling under $\mathrm{Ar}$ atmosphere, disclosing the formation of robust phosphorus-carbon $(\mathrm{P}-\mathrm{C})$ bonds and its important role for the excellent lithium storage capacity $\left(2786 \mathrm{~mA} \mathrm{~h} \mathrm{~g}^{-1}\right)$ and impressive stability $\left(80 \%\right.$ retention after 100 cycles). ${ }^{21}$ The unexpected renaissance of the BP material was then significantly revived in battery systems (Fig. 1b), including Li-ion battery, Na-ion battery, K-ion battery, Li-S battery, Zn-Ni battery, and all solidstate battery. Subsequently, its exploration in catalysis was also strengthened due to the large surface area, sufficient exposure of active sites, and tunable physicochemical properties.

In this review, we comprehensively summarize the progress of BP applied in energy storage and conversion (Fig. 2). Frequently used synthetic strategies and applications in battery systems, capacitor devices, and electro/photocatalysis are fully discussed. Moreover, an overview of the opportunities for future research within this rapidly developing field is profoundly put forward in time.

\section{Strategies for the preparation of bulk BP}

\subsection{Controlled pressure-assisted synthesis}

As illustrated in Fig. 3a, BP is a layered material, in which atomic layers are stacked together by the van der Waals interaction and the phosphorus atom is covalently bonded with three adjacent phosphorus atoms, forming a honeycomb structure. ${ }^{22}$ In 1914, Bridgman obtained BP under controlled

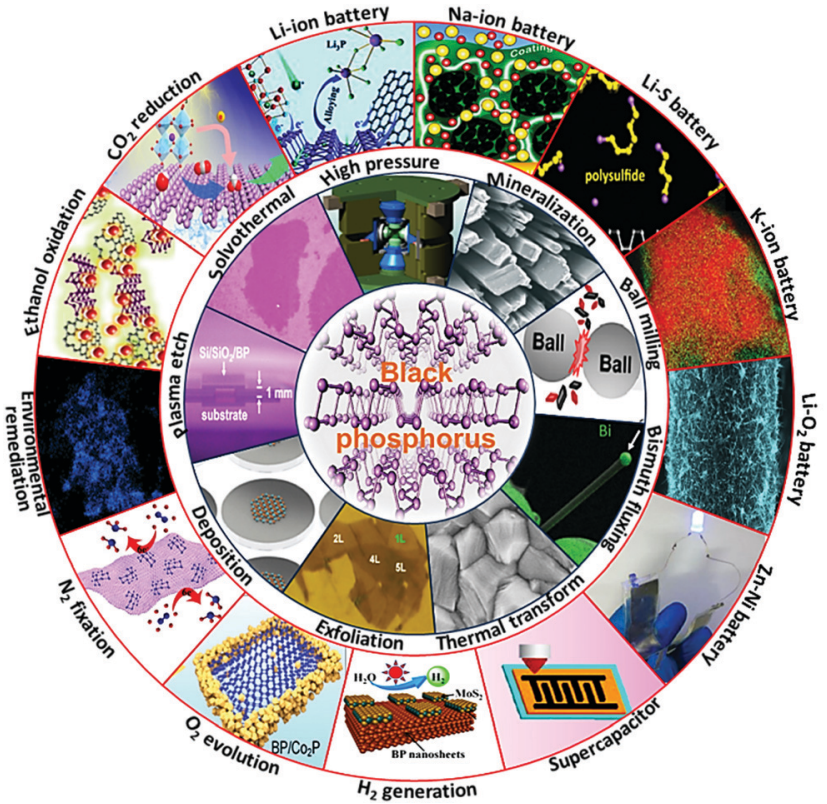

Fig. 2 Overview of the preparation and application of black phosphorus in energy storage and conversion area.

pressure. ${ }^{25}$ White phosphorus was firstly put into a hydrostatic column with a length of $15 \mathrm{~cm}$ and diameter of $1.5 \mathrm{~cm}$, and then heated at $200{ }^{\circ} \mathrm{C}$ and pressed at a high pressure of $1.2 \times$ $10^{9} \mathrm{~Pa}$. Keyes et al. increased the pressure to $1.3 \times 10^{9} \mathrm{~Pa}$ at the same temperature and collected a unique BP with a width of $0.1 \mathrm{~mm} .{ }^{26}$ Sun and his co-workers obtained orthorhombic BP from white $\mathrm{P}$ and red $\mathrm{P}$ powder in a cubic anvil high-pressure apparatus under the pressures of 2-5.0 GPa and temperatures of $200-800{ }^{\circ} \mathrm{C}^{27}$ When the pressure and temperature were set as high as $1.3 \times 10^{9} \mathrm{~Pa}$ and $800{ }^{\circ} \mathrm{C}$, respectively, BP was obtained in 1 hour by Dahbi and his co-workers. ${ }^{28}$ Zhang et al. also reported a kind of BP synthesized under a constant pressure of $10 \mathrm{kbar}$ by heating red phosphorus to $1000{ }^{\circ} \mathrm{C}$ and slowly cooling to $600{ }^{\circ} \mathrm{C}$ at a cooling rate of $100{ }^{\circ} \mathrm{C}$ per hour. ${ }^{22}$ It can be concluded that the controlled pressure-assisted synthetic approach has a good reproducibility and large-sized BP could be prepared in a short time. Nevertheless, this kind of high-pressure equipment needs special customization, which is not suitable for industrial processes at this stage.

\subsection{Mineralization agent-assisted synthesis}

Mineral additive-assisted method is a relatively mild approach for BP synthesis. It was proposed by Nilges et al. via the addition of small quantities of gold, tin, and tin iodide. ${ }^{3}$ The mineralization agent and red phosphorus were firstly mixed in a certain proportion and encapsulated in a quartz tube. After a series of heating processes, BP can be obtained with some unconverted red phosphorus and metal phosphide existing in the final product (Fig. 3b). ${ }^{3}$ Generally, tin phosphide can be formed close to the bulk residue by a mineralization mechanism, as illustrated in the reaction equation:

$$
\mathrm{Sn}_{3} \mathrm{P}_{4(\mathrm{~s})}+3 \mathrm{I}_{2(\mathrm{~g})} \rightleftharpoons 3 \mathrm{SnI}_{2(\mathrm{~g})}+\mathrm{P}_{4(\mathrm{~g})}
$$



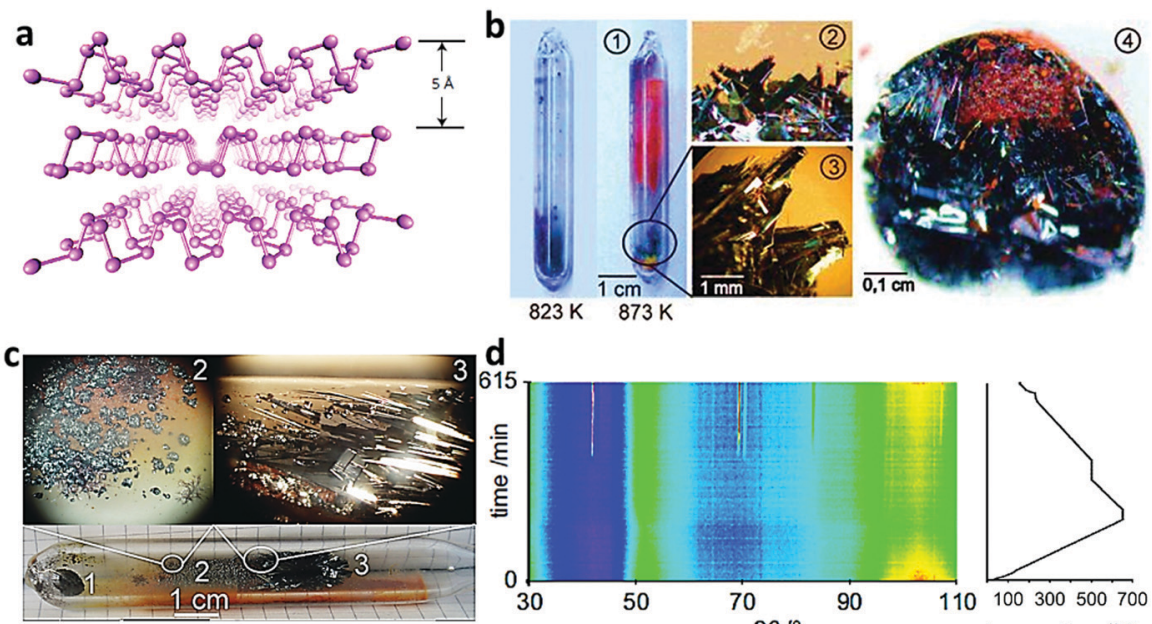

$873 \mathrm{~K}$
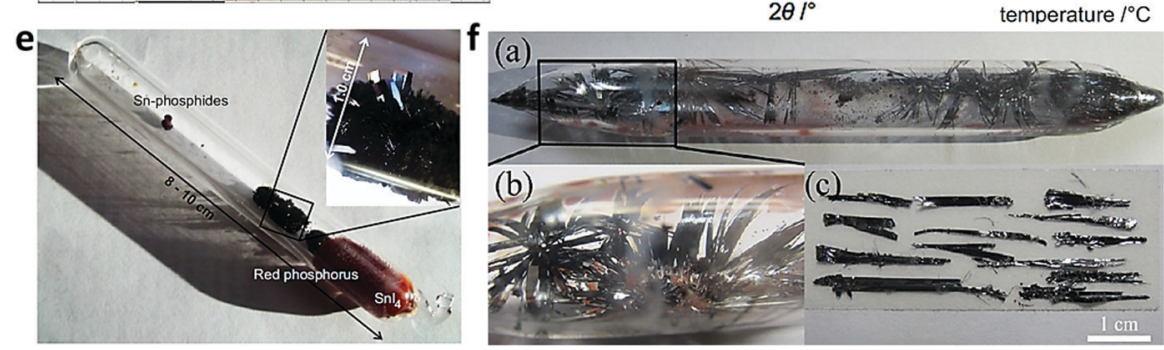

Fig. 3 Controlled pressure assisted and mineralization agent assisted synthesis of bulk BP. (a) Atomic structure of BP. Reproduced with permission ${ }^{22}$ Copyright 2014, Nature. (b) Silica ampules (picture 1) used for the preparation of BP (picture 3), Sn(Iv) iodide condensed on top of BP after thermal decomposition of $\mathrm{Au}_{3} \mathrm{SnP}_{7}$ at $1073 \mathrm{~K}$ (picture 2), and representative batch of $\mathrm{BP}$ grown on top of a $\mathrm{Au}_{3} \mathrm{SnP}_{7} / \mathrm{AuSn}$ bulk (picture 4). Reproduced with permission. ${ }^{3}$ Copyright 2007, American Chemical Society. (c) Silica ampoule after the reaction procedure with 1,2 and 3 representing the bulk residue, violet phosphorus and the main product BP. Reproduced with permission. ${ }^{23}$ Copyright 2008, Elsevier. (d) 2D-representation of the temperature-dependent in situ neutron diffraction experiment together with the applied temperature program. (e) Representative silica glass ampoule after the synthesis of BP with $\mathrm{Snl}{ }_{4}$ (orange) and red phosphorus (red) from the gas phase condensed at the right hand side of the ampoule. Reproduced with permission. ${ }^{24}$ Copyright 2014 , Elsevier. (f) Photo of the grown BP microribbons in ampule tube. Reproduced with permission. ${ }^{4}$ Copyright 2015, American Chemical Society.

Upon the mineralization process, they further optimized the synthetic conditions and produced large BP single crystals larger than $1 \mathrm{~cm}$ at low-pressure conditions in silica ampoules.

Fig. $3 \mathrm{c}$ shows the silica ampoule after the reaction procedure, in which 1,2 , and 3 represent the bulk residue, violet phosphorus, and the main product black phosphorus, and no impurity was found. ${ }^{23}$ In these designed procedures, $\mathrm{Au}$ is an essential part in the synthetic process, making it uneconomic for industrial applications. Thus, in 2014, Nilges and his co-workers optimized the synthesis and found that the single crystal BP could be grown by a short way transport reaction with red phosphorus as the precursor and $\mathrm{Sn} / \mathrm{SnI}_{4}$ as the mineralization additive. ${ }^{24}$ Based on the in situ neutron diffraction experiment together with the applied temperature program shown in Fig. 3d, the BP started to crystallize with an induction period of approximately $50 \mathrm{~min}$ and crystallized continuously during the cooling process. It can be clearly seen that $\mathrm{SnI}_{4}$ (orange) and red phosphorus (red) from the gas phase were condensed at the right hand side of the ampoule, while $\mathrm{BP}$ was formed in large bunches and excessive Sn forming Sn-Phosphides were present in small round spheres (Fig. 3e). ${ }^{24}$ Notably, it was reported by Zhao et al. that large size few-layered BP microribbons (Fig. 3f) could be obtained under atmospheric pressure by the delicate calcination of iodine, tin, and red phosphorus. ${ }^{4}$ Firstly, the silica glass ampoule was sealed under atmospheric pressure in the glovebox with iodine, tin, and red phosphorus inside. After loading in a muffle furnace, the temperature was slowly increased to $863 \mathrm{~K}$ and finally decreased to $393 \mathrm{~K}$, providing a conversion ratio of $97 \%$. When the temperature was increased to $673 \mathrm{~K}$, a light-yellow color appeared in the tube. At the same time, tin reacted with iodine to form tin iodide as follows:

$$
\mathrm{Sn}+\mathrm{I}_{2} \rightleftharpoons \mathrm{SnI}_{2}
$$

When the temperature was further increased from 673 to $863 \mathrm{~K}$, the RP gas reacts with tin iodide and tin gas to form the P-Sn-I compound, which worked as nucleation sites for the growth of BP microribbons. During the temperature decrease process, BP microribbons gradually crystallized and grew with the help of the mineralizer and this process lasted till $523 \mathrm{~K}$. It was revealed that the mineral additive-assisted method is an effective way for the synthesis of BP crystals. However, it is relatively tedious for the removal of impurities, which includes unreacted precursors and intermediate compounds.

\subsection{Bismuth fluxing method}

Bismuth fluxing method is a novel method for BP preparation. In 1981, Tanuma et al. successfully collected BP single crystals 
from a solution of white phosphorus in liquid bismuth. ${ }^{29}$ The white phosphorus purified by $15 \% \mathrm{HNO}_{3}$ and water steam and bismuth were set separately in two branches of a glass tube, which was then sealed. After the melted bismuth was poured onto white phosphorus and shaken well, the mixture was heat-treated and washed with $\mathrm{HNO}_{3}$ before BP collection. However, it should not be ignored that white phosphorus is highly flammable and toxic. Morita's group improved the bismuth flux method and relatively stable red phosphorus was applied to promote the synthetic safety. ${ }^{1}$ Specifically, red phosphorus and bismuth were set separately in a homemade glass tube and melted bismuth was then poured on the white phosphorus formed during red phosphorus calcination. After $\mathrm{HNO}_{3}$ washing, needle-shaped and film- or plate-shaped BP could be obtained. It is apparent that the conversion of red phosphorus and the formation of BP were all in an evacuated quartz-glass apparatus, effectively avoiding the risk during the BP preparation.

\subsection{Mechanical milling}

Mechanical milling is a solid-state powder processing technique involving repeated welding, fracturing, and rewelding of powder particles in a high-energy ball mill. ${ }^{31}$ Sohn and co-workers prepared BP by means of high energy mechanical milling at ambient temperature and pressure, using red phosphorus as the precursor and argon as the protective atmosphere. ${ }^{2}$ Tatsumisago et al. further compared the quality of $\mathrm{BP}$ prepared from red phosphorus through mixer milling and planetary ball milling, and found that BP prepared using the mixer milling has much higher crystallinity due to the efficient impact interaction for reaction from red phosphorus to BP (Fig. 4a). ${ }^{30}$ Fig. $4 \mathrm{~b}$ exhibits the composites composed of BP and acetylene black obtained by milling in the mixer mill apparatus, and it includes smaller secondary particles with a size of $1-5 \mu \mathrm{m}$, which is much smaller than that of bare BP $(c a .30 \mu \mathrm{m}) .^{30}$

\section{Process of BP for applications}

Owing to the great influence of the thickness on the electronic, optical, and thermal properties of the BP material, various treatment methods, such as mechanical exfoliation, liquid exfoliation, electrochemical exfoliation, and deposition, have been rationally explored to achieve few-layered BP active materials for further applications.

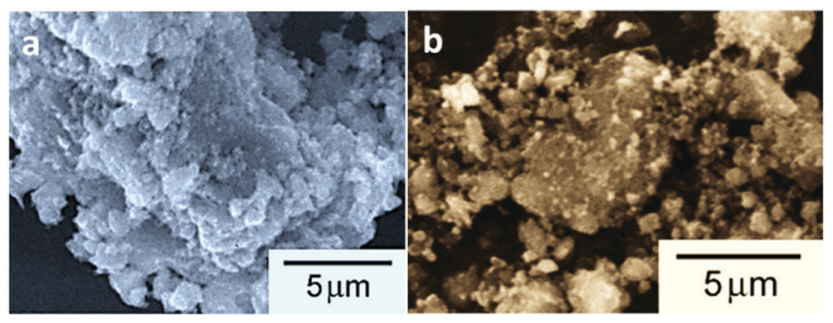

Fig. 4 (a) Morphology of BP and (b) the ball-milled BP and the acetylene black composite. Reproduced with permission. ${ }^{30}$ Copyright 2010, Elsevier.

\subsection{Exfoliation treatment}

The exfoliation process is a popular strategy for property enhancement, which can be generally divided into mechanical exfoliation, liquid exfoliation, and electrochemical exfoliation. Mechanical exfoliation is a traditional method for preparing 2D nanomaterials from bulk crystals, which was used for the first time by Geim et al. for the exploration of graphene. ${ }^{40} \mathrm{Lu}$ et al. prepared few-layer BP material by mechanical exfoliation onto the silicon substrate with an $\mathrm{SiO}_{2}$ capping layer and then, $\mathrm{Ar}^{+}$ plasma was applied to thin down the sheets. ${ }^{32}$ Compared to the thin sheets of BP after mechanical exfoliation (Fig. 5a), the thickness of the plasma-treated BP film decreases markedly and some of the regions become single layer (Fig. 5b). In addition, the structure of the plasma-treated BP film was not destroyed, which is beneficial for further application. Unfortunately, the shortcomings of mechanical exfoliation is also obvious, such as the time-consuming process and uncontrolled quality.

In order to improve the production efficiency, liquid phase exfoliation has recently attracted great attention. When the solvent molecules are inserted in bulk BP, the surface tension effectively increases the interlayer space, promoting the BP exfoliation during ultrasonic treatment. In addition, electrochemical exfoliation is a promising way for the production of high-quality nanometer BP as ions can be driven by the electric field to intercalate into the layered bulk material, expanding the volume and decreasing the van der Waals forces. Owing to its excellent efficiency, mild reaction conditions, simple operation, and environment friendliness, this method has attracted great attention. O'Brien and his co-workers produced few-layered BP by liquid exfoliation, whose shapes and sizes were revealed by atomic force microscopy (AFM) (Fig. 5c). ${ }^{33}$ It shows that nanosheets as large as ca. $200 \mathrm{~nm} \times 200 \mathrm{~nm}$ maintain a thickness of 3.5-5 nm, predominantly consisting of three to five layers. ${ }^{41}$ Sun et al. synthesized BP quantum dots (BPQDs) using a liquid exfoliation method combining probe sonication and bath sonication (Fig. 5d). ${ }^{34}$ Typically, the probe sonication of bulk BP was carried out before ice bath sonication in 1-methyl-2-pyrrolidone (NMP) and then, PEG was selected to improve their stability in water. With the help of NMP, Zhang et al. achieved a steady yield of BP nanosheets by ultrasonication treatment of bulk BP at a temperature below room temperature, followed by the removal of the residual bulk BP by centrifugation and washing (Fig. 5e). ${ }^{35}$ Hersam et al. presented a scalable method for the preparation of pristine 2D BP nanosheets via direct liquid exfoliation in organic solvents, in which sealed-tip ultrasonication system was employed to trigger the production of stable BP dispersions. ${ }^{36}$ Based on the schematic BP crystal structure shown in Fig. 5f, there are A and B high symmetry directions, and the perspectives of these two directions in the transmission electron microscopy (TEM) images are consistent with the BP crystal structures. Salehi-Khojin et al. prepared high-quality 2D BP with a thickness ranging from 5.8 to $11.8 \mathrm{~nm}$ (Fig. 5g) through the sonication of bulk BP in a Sonics Vibra-Cell sonicator, centrifugation and filtration of the top part of the solution. ${ }^{37}$ To further identify the reliable quality, the nanoflakes were analyzed by performing energy-dispersive X-ray spectroscopy (EDX) analysis, which indicated that the obtained 

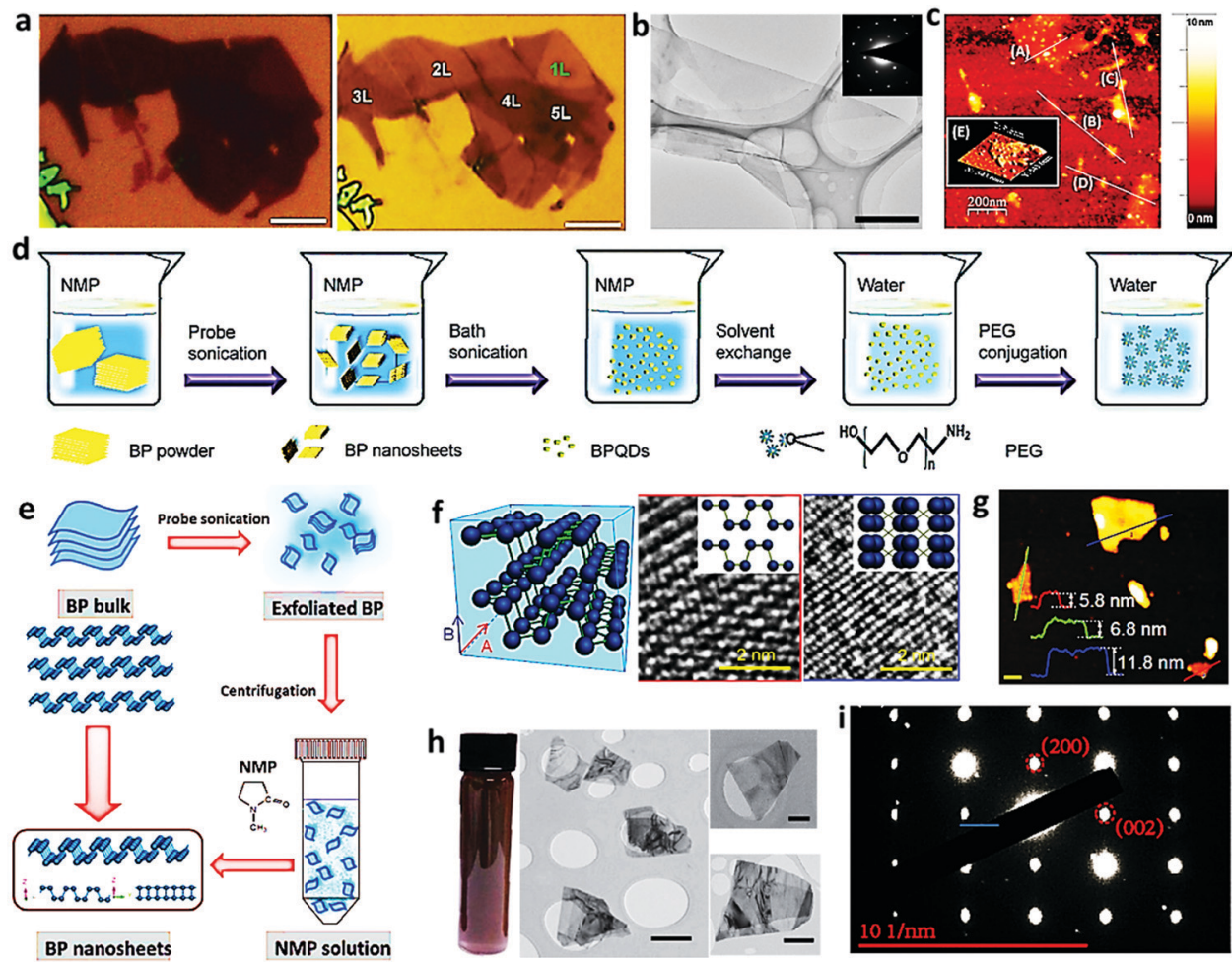

Fig. 5 Exfoliation treatment of bulk BP for further application. (a) Optical image of multilayered pristine (left) and $\mathrm{Ar}^{+}$sputtered (right) BP (scale bar: $5 \mu \mathrm{m})$. (b) TEM image of the exfoliated BP nanosheet (scale bar: $500 \mathrm{~nm}$ ). Reproduced with permission. ${ }^{32}$ Copyright 2014, Springer. (c) AFM height profile image. Reproduced with permission. ${ }^{33}$ Copyright 2014, Royal Society of Chemistry. (d) Synthesis and surface modification of the BP quantum dots. Reproduced with permission. ${ }^{34}$ Copyright 2015, Wiley. (e) Schematic illustration of solution-phase exfoliation route to prepare few-layer BP nanosheets. Reproduced with permission. ${ }^{35}$ Copyright 2017, Wiley. (f) TEM images of solvent-exfoliated BP nanosheets along the A and B directions. Reproduced with permission. ${ }^{36}$ Copyright 2015, American Chemical Society. (g) AFM image of the individual BP nanoflake obtained by liquid-phase exfoliation. Reproduced with permission. ${ }^{37}$ Copyright 2015, Wiley. (h) Photograph of the dispersion of exfoliated BP and its corresponding low-resolution TEM image. Reproduced with permission. ${ }^{38}$ Copyright 2015, Nature. (i) SAED pattern of the exfoliated BP sheets using [HOEMIM][TfO]. Reproduced with permission. ${ }^{39}$ Copyright 2015, American Chemical Society.

nanosheets were only composed of phosphorus, without any noticeable impurity components.

As BP is known to be unstable, which is easily degradable via reaction with water and oxygen, the facile protective way is urgently needed for the further application of BP. Coleman and his co-workers reported that exfoliated few-layered BP (Fig. 5h) could be protected in N-cyclohexyl-2-pyrrolidone (CHP) solvent and the solvation shell acted as a barrier to prevent oxidative species reaching the nanosheet surface. ${ }^{38}$ Controlled centrifugation was also established in this work as the size-selection technique, which is of great importance, as some applications, such as mechanical reinforcement, benefit from large flakes, while others, such as catalysis, prefer small sheets. ${ }^{42,43}$ Notably, an alternative pathway $\left(\mathrm{BP}+3 \mathrm{H}_{2} \mathrm{O} \rightarrow \mathrm{BP}_{2 \mathrm{VAC}}+\mathrm{PH}_{3}+\mathrm{H}_{3} \mathrm{PO}_{3}\right)$ involving nanosheet edges was also suggested as acid-base disproportionation reaction involving nanosheet edges may become dominant when basal plane reactions are suppressed. Ionic liquids, a kind of molten salts possessing many excellent properties (e.g., non-volatility, high thermal stability, high viscosity, high ionic conductivity, nontoxicity, versatile solubility, and solvent recyclability), have been reported to play an effective role in exfoliating 2D materials, including graphene or the $\mathrm{MoS}_{2}$ nanosheet. ${ }^{44-46}$ Mu's group developed a facile and large-scale liquid-exfoliation method to produce stable and high-concentration dispersions of BP nanosheets using ionic liquids, which could be stabilized without any obvious sedimentation and aggregation in ambient air for one month. The high-quality single-crystal BP with orthorhombic crystalline character (Fig. 5i) could surprisingly achieve an ultrahigh concentration in 1-hydroxyethyl-3-methylimidazolium trifluoromethanesulfonate [HOEMIM] [TfO] ionic liquid, providing a feasible way for the commercialization of BP. ${ }^{39}$ In 2018 , Zeng's group also prepared monolayer BP dots through the exfoliation treatment of single crystalline BP powder in ice-bath ultrasonication and subsequent gradient high-speed centrifugation. ${ }^{47}$ They further simplified the exfoliation procedure of BP by the vigorous stirring of $\mathrm{BP} / \mathrm{NaOH}$ under the protection of an inert atmosphere. After the solution was gradually turned to light yellow, stepwise centrifugation was then utilized to purify the exfoliated $\mathrm{BP} .^{48}$ Although exfoliation is a widely accepted facile treatment for the application of modified BP in energy storage and conversion, the batch-to-batch quality is still challenging to precisely control and is needed to be further optimized.

\subsection{Deposition process}

In general, apart from the exfoliation treatment, the vapor deposition method is another effective process for the 
preparation of 2D BP nanomaterials. Based on the direct chemical synthesis of 2D BP from phosphorus precursors, large-area ultrathin 2D nanomaterials with excellent crystal quality, tunable thickness, and size can be obtained. In 2016, Smith et al. directly synthesized 2D BP from red phosphorus on a silicon substrate through the deposition method. ${ }^{49}$ First, red phosphorus and tin powder were purified by rinsing and shaking in $\mathrm{NaOH}$ solution. After the red phosphorus thin film was obtained on a silicon substrate by heating the precursor powder (Fig. 6a), it was then transferred into the centrifuge tube and heated together with $\mathrm{Sn}$ and $\mathrm{SnI}_{4}$, forming the BP film on the substrate. ${ }^{49}$ Pulsed-laser deposition method was utilized by Lau's group to explore amorphous BP (a-BP) ultrathin films with a highly disordered structure at a low temperature (Fig. 6b). When applied in a proof-of-concept FET device, a typical p-type transistor behavior was observed, verifying the hole as the dominating charge carrier. ${ }^{50}$ In addition, Xia et al. obtained crystalline BP on sapphire substrates by phase conversion from red to black phosphorus upon calcination (Fig. 6c), during which a homemade setup was applied for thermal evaporation (Fig. 6d). ${ }^{51}$ The obtained BP film with a domain size ranging

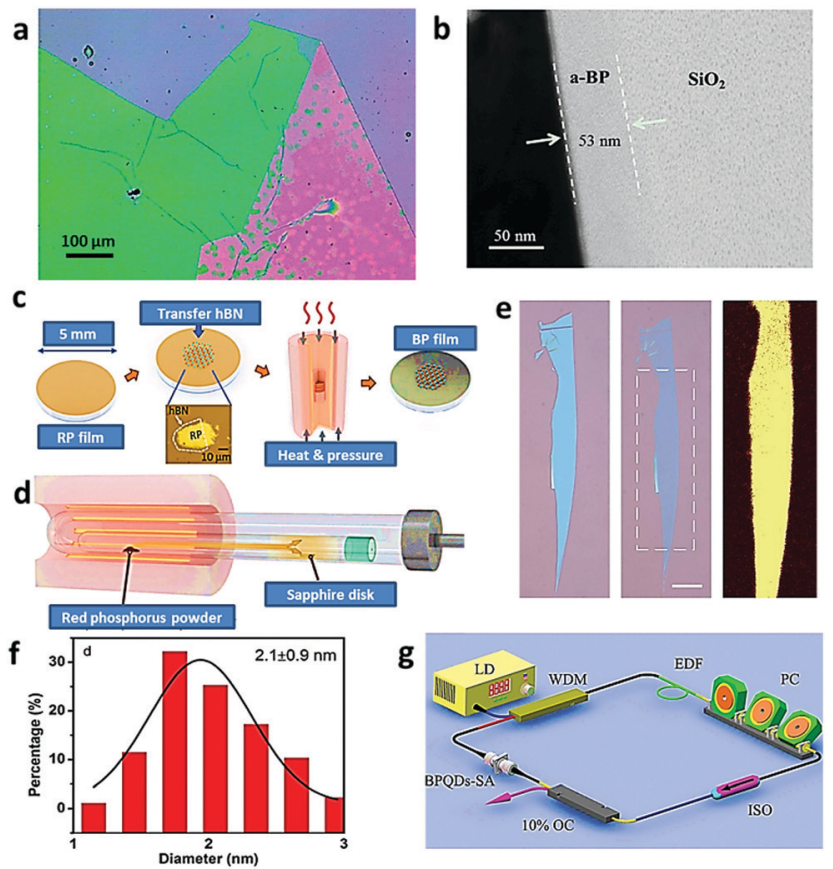

Fig. 6 Deposition synthesis of few-layered BP for further application. (a) Optical images of amorphous red phosphorus films grown from vapor deposition with noticeable folds. Reproduced with permission. ${ }^{49}$ Copyright 2016, IOP Publishing. (b) Cross-sectional TEM images of BP thin film. Reproduced with permission. ${ }^{50}$ Copyright 2015, Wiley. (c) BP synthesis process flow and (d) schematic of the homemade setup for red phosphorus thin film deposition on a sapphire disk. Reproduced with permission. ${ }^{51}$ Copyright 2018, Wiley. (e) Optical image of a thick BP flake when it was exfoliated and thinned down to $2 \mathrm{~L}$ by $\mathrm{O}_{2}$ plasma etching (scale bar, $10 \mathrm{~mm}$ ) with $\mathrm{PL}$ mapping image of the area marked by a dashed rectangle integrated. Reproduced with permission. ${ }^{52}$ Copyright 2016, Nature. (f) Statistical analysis of the lateral sizes of BPQDs and (g) schematic of the passively mode-locked fiber laser based on the BPQDs saturable absorber. Reproduced with permission. ${ }^{53}$ Copyright 2016, Wiley. from 40 to $70 \mu \mathrm{m}$ showed a field-effect mobility of $160 \mathrm{~cm}^{2} \mathrm{~V}^{-1} \mathrm{~s}^{-1}$ along the armchair direction at room temperature and $200 \mathrm{~cm}^{2} \mathrm{~V}^{-1} \mathrm{~s}^{-1}$ at about $90 \mathrm{~K}^{51}$ The deposition method provides the possibility of preparing various BP materials with good quality and stable performance. Notably, the high cost and strict requirement of the deposition equipment still significantly hinder its large-scale application. Besides, the combustible gas detection devices are indispensable to avoid possible hazards.

\subsection{Plasma etching}

Apart from exfoliation and deposition, there are other methods that also significantly influence the properties of BP and promote its further utilization. For example, Lu et al. proposed $\mathrm{O}_{2}$ plasma etching as an effective treatment to thin down thickexfoliated BP flakes layer-by-layer with atomic precision. This method can also engineer the defects in a BP monolayer, triggering new photon emissions and modulating the doping level. ${ }^{52}$ During the $\mathrm{O}_{2}$ plasma treatment, the top layer of the BP flake was firstly oxidized to $\mathrm{P}_{x} \mathrm{O}_{y}$ and with the penetration of the $\mathrm{O}_{2}$ plasma, the deeper BP layer could be further oxidized and the upper $\mathrm{P}_{x} \mathrm{O}_{y}$ would be physically sputtered away, leading to the precisely fabricated BP sheet with a designated number of layers. As shown in Fig. 6e, the thick BP flake (left) was precisely thinned down to $2 \mathrm{~L}$ (middle) by $\mathrm{O}_{2}$ plasma etching, which is quite uniform and of high quality, as investigated by the integrated photoluminescence (PL) mapping image (right). Nevertheless, this method could not be widely applied due to its relatively high cost and complicated operation techniques.

\subsection{Solvothermal process}

The solvothermal method was adopted by $\mathrm{Yu}$ et al. to prepare BP QDs from bulk BP, which is facile and controllable to make it possible to produce the target sample at a large scale compared with the previously reported methods. ${ }^{53}$ Starting from bulk BP grinding, BP powder was then vigorously stirred in saturated $\mathrm{NaOH}$ NMP solution under the protection of an inert atmosphere, resulting in uniform QDs with an average lateral size of $2.1 \pm 0.9 \mathrm{~nm}$ (Fig. 6f). A passively mode-locked fiber laser based on the BP QD absorber has been designed, which demonstrates the generation of an ultrashort pulse in an Er-doped fiber laser (Fig. 6g). ${ }^{53}$ The solvothermal reaction was also investigated by Yoshida and coworkers for the synthesis of BP with amines and RP as the solvent and the starting material, respectively. ${ }^{54}$ The effects of the reaction time, particle size of $\mathrm{RP}$, and RP concentration in the slurry on the yield of BP were evaluated. Encouragingly, the yield of the BP could be saturated above $15 \mathrm{~g} \mathrm{~L}^{-1}$. This research provides highly valuable exploration for further scalable preparation. Moreover, Xiao et al. also proposed the facial one-step solvothermal synthesis of BP nanosheets from relatively safe RP. ${ }^{55} \mathrm{RP}$ was first ground in mortar and then put in an autoclave with ethylenediamine as the solvent. When the temperature was higher than $165{ }^{\circ} \mathrm{C}$, no diffraction peak for RP was observed, indicating the complete transition of RP to BP. Notably, it could be up to $2.1 \mathrm{~g}$ for one batch and the efficiency of phase transition was up to $100 \%$. Thus, the solvothermal-based process exhibits highly impressive 
advantages during the synthesis of $\mathrm{BP}$, such as facile procedure, low cost, high yield, and desirable transition. Although the solvothermal process shows good performance in the modification of BP, it also has significant drawbacks, such as indiscernible reaction process and high quality requirement of the reaction vessel.

\section{Application in energy storage devices}

\subsection{Secondary batteries}

4.1.1 Lithium ion battery. BP with high theoretical specific

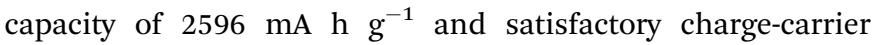
mobility has great potential for application in lithium ion battery. ${ }^{63}$ A unique 3D chemical cross-linking structure of the BP@CNTs hybrid was proposed by Han's group, in which CNT was selected to play the role of the conductive channel and support the framework. BP was anchored in the CNT matrix through chemical crosslinking and electrostatic adsorption bond, leading to enhanced transport of electron and ions and mitigated volume change of the BP during charge and discharge. ${ }^{56}$ Fig. 7a compares the anode charge transport process for the bare multilayered BP (left) and the designed BP@CNTs hybrid (right). Bare BP layers maintain a long and a slow-track pathway, whereas the cooperating CNTs could provide a short and fast-track path along the conductive tube. The obtained BP@CNT composite anode gives impressive reversible capacity (1088 $\left.\mathrm{mA} \mathrm{h} \mathrm{g}{ }^{-1}\right)$, good conductivity (88.9 $\Omega$ ), and satisfactory cycling stability (757.3 $\mathrm{mA} \mathrm{h} \mathrm{g}^{-1}$ after 650 cycles), showing great potential for application in energy storage batteries. ${ }^{56}$ In order to have a deeper understanding of the binding and diffusion behavior of lithium in the BP-based anode, Zhang et al. carried out the density functional theory calculation (DFT). The adsorption of Li was firstly performed by loading lithium atoms on the surface and then diffusing along the armchair or the zigzag direction (Fig. 7b). ${ }^{57}$ Surprisingly, in spite of the large energy barrier $(0.68 \mathrm{eV})$ along the armchair direction, the energy barrier along the zigzag direction is as low as $0.08 \mathrm{eV}$ in the monolayer $\mathrm{BP}$, much lower than those in graphene and $\mathrm{MoS}_{2}$. In addition, the average voltage for lithium intercalation was calculated to be about $2.9 \mathrm{~V}$, which is promising for applications in lithium ion batteries with high voltage and rate capability. ${ }^{57}$ The lithium storage capability of phosphorus, an abundant low-cost abundant material, could be significantly enhanced through intensive mechanical milling, in which BP was transformed into small particles (ca. $100 \mathrm{~nm}$ ). The obtained BP provided remarkable initial lithiation and delithiation capacities of 2010 and $1814 \mathrm{~mA} \mathrm{~h} \mathrm{~g}^{-1}$, respectively. ${ }^{2}$ However, there is usually fast capacity decay due
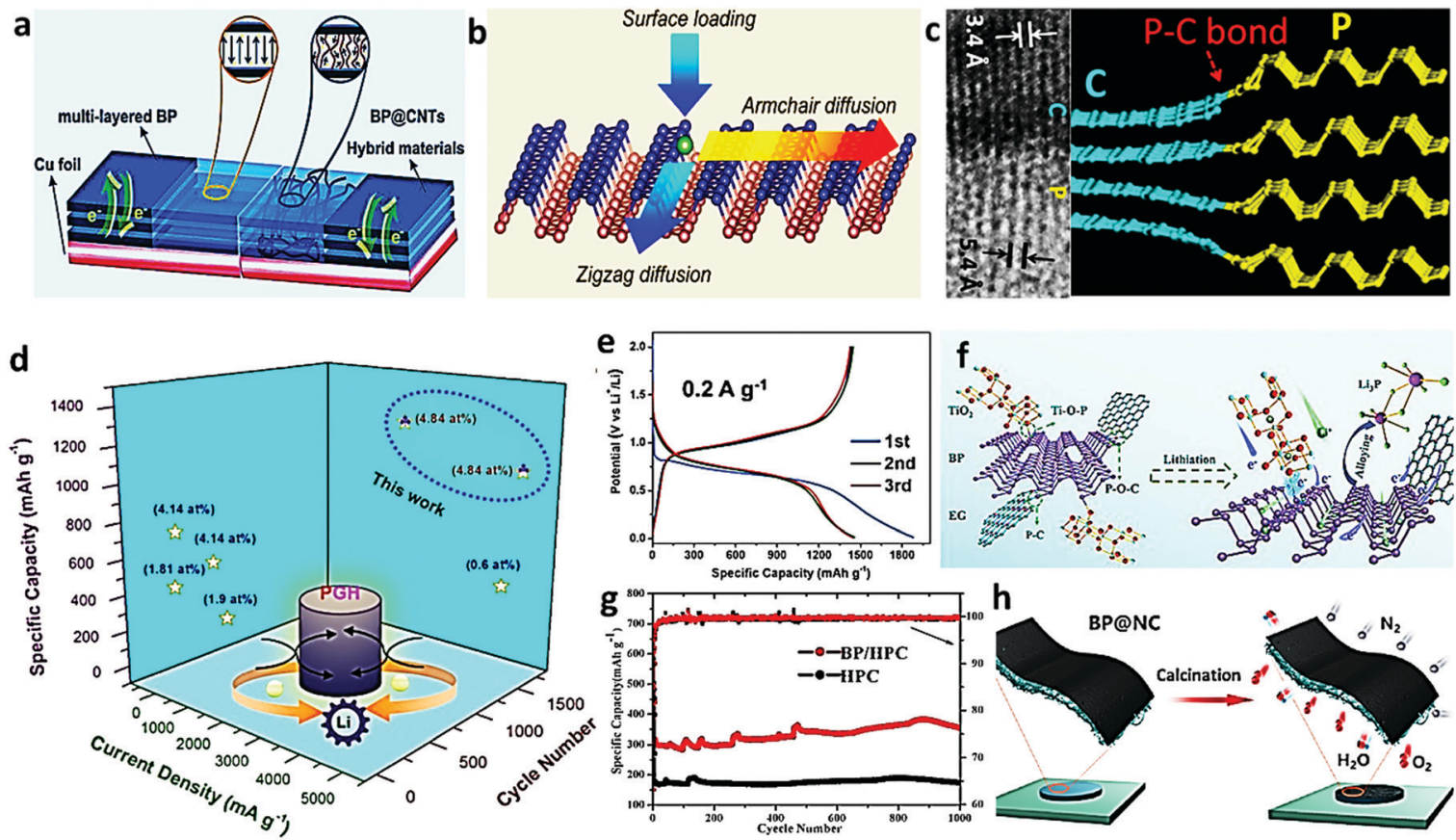

Fig. 7 Application of engineered BP in lithium-ion battery. (a) Illustration of BP and BP@CNTs hybrids samples as the LIB anodes. Reproduced with permission. ${ }^{56}$ Copyright 2020, Wiley. (b) Schematic of Li surface loading and diffusion along armchair and zigzag directions on the BP surface. Reproduced with permission. ${ }^{57}$ Copyright 2015, American Chemical Society. (c) HRTEM image of the BP-G composite and schematic of the generated $\mathrm{P}-\mathrm{C}$ bond. Reproduced with permission. ${ }^{21}$ Copyright 2014, American Chemical Society. (d) Comparison of the specific capacity, cycling stability, and the corresponding phosphorus concentration between the PGH electrode and some previous works. Reproduced with permission. ${ }^{58}$ Copyright 2020 , American Chemical Society. (e) Galvanostatic charge-discharge profile of the P-KB composite. Reproduced with permission. ${ }^{59}$ Copyright 2019 , Elsevier. (f) Schematic demonstration of the lithiation process of the prepared $\mathrm{BP}_{-} \mathrm{TiO}_{2}-\mathrm{C}$ composite. Reproduced with permission. ${ }^{60} \mathrm{Copyright} 2019$, Elsevier. (g) Cycle performances (current density $=1000 \mathrm{~mA} \mathrm{~g}^{-1}$ ) of BP/HPC and HPC. Reproduced with permission. ${ }^{61}$ Copyright 2020, Wiley. (h) Schematic illustration of the free-standing BPaNC anodes. Reproduced with permission. ${ }^{62}$ Copyright 2020, American Chemical Society. 
to the large volume change (300\%) during the formation of $\mathrm{Li}_{3} \mathrm{P}$. Cui et al. demonstrated a strategy for the synthesis of BP-graphite (BP-G) composite anodes with robust phosphorus-carbon (P-C) bonds, which gave an initial specific capacity of 2786/ $2382 \mathrm{~mA} \mathrm{~h} \mathrm{~g}^{-1}$ during the lithiation/delithiation process, corresponding to a coulombic efficiency of $85 \%$, which is much higher than that of the BP/graphite mixture (58\%). ${ }^{21}$ Apparently, the HRTEM image of BP-G exhibits two different parallel lines. The lattice lines with a smaller spacing $(0.34 \mathrm{~nm})$ are related to the gap in graphite, while the larger ones $(0.54 \mathrm{~nm})$ are related to the BP layers (Fig. 7c). The coherent $\mathrm{P}-\mathrm{C}$ bonding could be found at the boundary of two different regions and was further verified in XPS analysis. ${ }^{21}$ This research paved a novel way for the formation of the $\mathrm{P}-\mathrm{C}$ bond through facile mechanical milling, which promotes effective contact between phosphorus and carbon. Sun et al. further enriched the carbon-phosphorus bonds by sacrificing 2D BP on the graphene skeletons through one-step hydrothermal treatment for high-performance lithiumion storage (1000 $\mathrm{mA} \mathrm{h} \mathrm{g}^{-1}$ after 1700 cycles) (Fig. $\left.7 \mathrm{~d}\right){ }^{58}{ }^{5}$ The C-P bond was confirmed in the FT-IR investigation and a negative shift of the $\mathrm{C}=\mathrm{C}$ skeleton peak from the original 1620 to $1560 \mathrm{~cm}^{-1}$ was attributed to the conjugative effect between carbon and phosphorus, further indicating the successful implantation of phosphorus in the carbon network. In addition, the 3D composite possesses abundant open channels and wide pore size distribution, which is beneficial for ion-diffusion promotion and reaction kinetics enhancement. Although phosphorus has large abundance and high theoretical capacity, its high dispersion in the carbon matrix is always a key point for the fulfillment of high lithium storage capacity. Lu's group rationally designed a facile and cost-effective strategy based on high-energy mechanical milling (1000 rpm for $24 \mathrm{~h}$ in total) for the preparation of BP nanoparticles within the carbon matrix. Red phosphorus could be transformed into BP at the ambient temperature and pressure and well dispersed in the carbon matrix, providing impressive electrochemical performance both in the half cell $\left(1000 \mathrm{~mA} \mathrm{~h} \mathrm{~g}{ }^{-1}\right.$ after 300 cycles $)$ and the full cell $(92 \%$ after 200 cycles at $0.5 \mathrm{C}) .{ }^{59}$ Nevertheless, as illustrated in Fig. 7e, the first lithiation and delithiation capacity are $1882 \mathrm{~mA} \mathrm{~h} \mathrm{~g}^{-1}$ and $1449 \mathrm{~mA} \mathrm{~h} \mathrm{~g}^{-1}$, respectively, achieving an initial Coulombic efficiency (ICE) of $76.4 \%$, which is not high enough for the practical full cell. Apart from the P-C bond, Zhu's group also found that the strong Ti-O-P bond, formed between $\mathrm{TiO}_{2}$ and $\mathrm{BP}$, could further improve the utilization of the active material and the transport of interfacial electron (Fig. 7f). The amorphous $\mathrm{BP}-\mathrm{TiO}_{2}-\mathrm{C}$ nanocomposite fabricated by the facile ball milling process delivered impressive electrochemical properties, e.g., high reversible capacities ( $935.4 \mathrm{~mA} \mathrm{~h} \mathrm{~g}^{-1}$ ) and good cycling stabilities $\left(85.3 \%\right.$ after 300 cycles at $\left.2 \mathrm{~A} \mathrm{~g}^{-1}\right){ }^{60}$ Based on X-ray photoelectron spectra (XPS), the Ti-O-P bond with bridging oxygen bonds formed between $\mathrm{BP}$ and $\mathrm{TiO}_{2}$ was clearly identified. This might result from the reaction between the hydroxyl groups on the BP surface and the hydroxyl groups of $\mathrm{TiO}_{2}$, which was promoted by the remarkably increased interface temperature as a result of intensive collisions between the balls. ${ }^{64}$ Generally, hollow porous carbon (HPC) has a large specific surface area and a porous structure, which can act as the ideal matrix for BP. Zhou et al. firstly prepared HPC by hydrothermally treating sodium dodecyl sulfate and glucose dissolved in water and then synthesized the BP/HPC hybrid composite through solvothermal treatment. ${ }^{61}$ Compared to bare HPC, the synthetic composite material (BP/HPC) has a specific capacity of $300 \mathrm{~mA} \mathrm{~h} \mathrm{~g}^{-1}$, approximately twice as high as that of HPC (170 $\left.\mathrm{mA} \mathrm{h} \mathrm{g}^{-1}\right)$ (Fig. 7g). In addition, there will be a slight capacity increase from $300 \mathrm{~mA} \mathrm{~h} \mathrm{~g}^{-1}$ to $350 \mathrm{~mA} \mathrm{~h} \mathrm{~g}{ }^{-1}$ even after 1000 cycles, indicating its great potential as commercial lithiumion battery anode materials. Recently, Wu et al. further applied BP nanosheets in the fabrication of the free-standing flexible anode together with nanocellulose (NC) nanowires (10.2\% elongation) via the facile vacuum-assisted filtration approach, offering a threedimensional (3D) mixed-conducting network for ion transports (Fig. 7h). ${ }^{62}$ Using molecular dynamic simulations and DFT calculations, Wu et al. revealed the greatly enhanced $\mathrm{Li}^{+}$diffusion in the BP@NC composite and relieved the repulsive force at the $\mathrm{BP} / \mathrm{NC}$ interface. Benefiting from the synergistic effect of BP sheets and multifunctional nanocellulose, the BP@NC electrodes demonstrated excellent lithium storage capabilities (1020.1 $\mathrm{mA} \mathrm{h} \mathrm{g}^{-1}$ at $0.1 \mathrm{~A} \mathrm{~g}^{-1}$ and $994.4 \mathrm{~mA} \mathrm{~h} \mathrm{~g}^{-1}$ at $0.2 \mathrm{~A} \mathrm{~g}^{-1}$ ) and moderate cycling stability ( $c a$. 85\% retention after 400 cycles), verifying the promising potential of cellulose employment to prepare high-performance flexible anodes for advanced lithium ion battery. ${ }^{62}$

Among various carbon materials, metal-organic frameworks (MOFs) are porous nanomaterials with abundant micropores and large surface, and are thus regarded as appealing candidates to address the large volume change, electrode pulverization, and sluggish ion penetration. ${ }^{72,73}$ Zhou et al. rationally designed a kind of 2D BP/NiCo MOF hybrid through the sonication of the as-obtained few-layer $\mathrm{BP}$ nanosheets, $\mathrm{CoCl}_{2}, \mathrm{NiCl}_{2}$, and benzenedicarboxylic acid (BDC). ${ }^{65}$ The BP with sheet-like morphology was utilized as the scaffold to anchor the NiCo MOF through a facile solution reaction, as shown in Fig. 8a. The carboxylate groups in $\mathrm{BDC}^{2-}$ chelating with metal ions and bonding with $\mathrm{BP}$ simultaneously could result in a stable hybrid structure with sufficient redox active sites, leading to enhanced electrochemical performance with good reversible capacity $\left(569 \mathrm{~mA} \mathrm{~h} \mathrm{~g}{ }^{-1}\right.$ at $2 \mathrm{~A} \mathrm{~g}^{-1}$ after 250 cycles) and excellent rate capability (398 $\mathrm{mA} \mathrm{h} \mathrm{g}^{-1}$ at $5 \mathrm{~A} \mathrm{~g}^{-1}$ after 1000 cycles). As a promising candidate of anode materials for lithium-ion battery, its electrochemical performance is remarkably influenced by the precursor and the synthetic conditions. To have a close insight into the electrochemical activity of pure BP under different pressures and temperatures, Xie et al. systematically prepared various orthorhombic BP in a cubic anvil high-pressure apparatus with a cubic sample chamber $(23 \mathrm{~mm}$ edges) under different pressures (2-5.0 GPa) and temperatures $\left(200-800{ }^{\circ} \mathrm{C}\right)$ for $15 \mathrm{~min} .{ }^{27}$ When white phosphorus was utilized as the precursor, the initial lithiation and delithiation capacities could reach 2505 and $1354 \mathrm{~mA} \mathrm{~h} \mathrm{~g}^{-1}$ at $4 \mathrm{GPa}$ and $400{ }^{\circ} \mathrm{C}$, which was a little lower than that using red phosphorus as the precursor at $4.5 \mathrm{GPa}$ and $800{ }^{\circ} \mathrm{C}\left(2649\right.$ and $\left.1425 \mathrm{~mA} \mathrm{~h} \mathrm{~g}^{-1}\right)$. It is noteworthy that in the (020) crystal plane of BP, the largest space for $\mathrm{Li}^{+}$ intercalation is along the $a$-axis, while for the (040) crystal plane, 
a

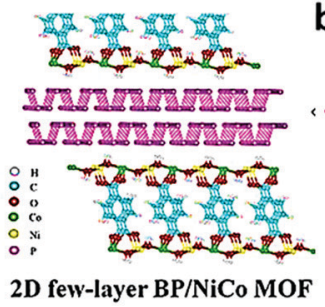

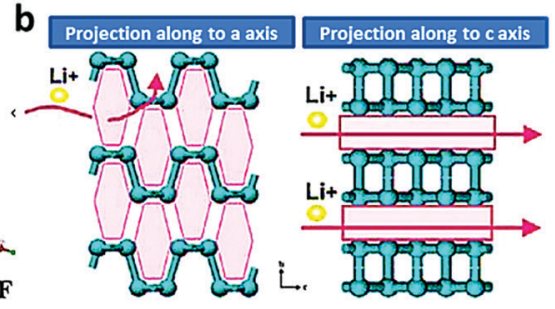
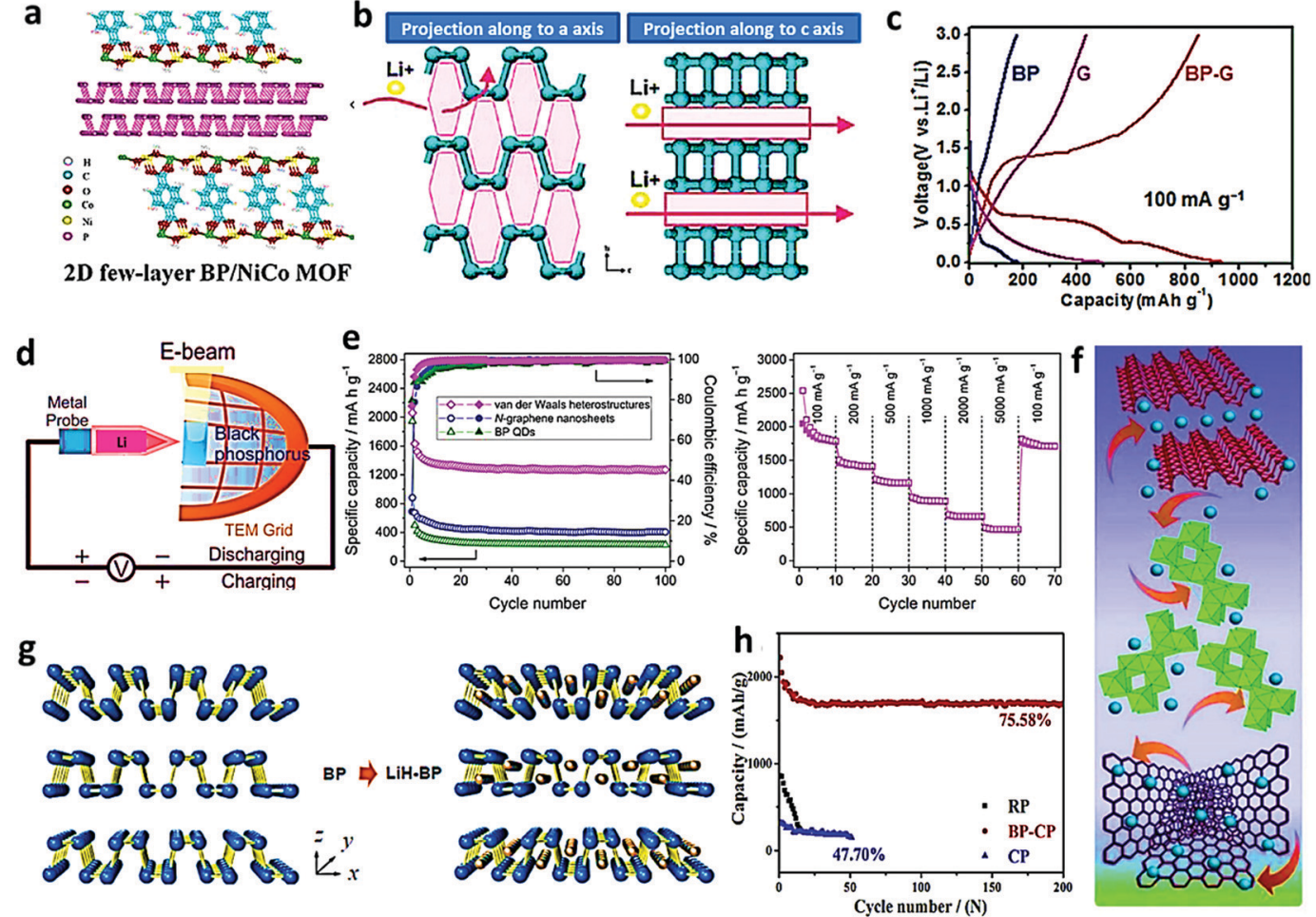

Fig. 8 Application of BP-based electrodes in the lithium-ion battery. (a) Illustration of the 2D BP/NiCo MOF hybrid. Reproduced with permission. ${ }^{65}$ Copyright 2019, Royal Society of Chemistry. (b) Projections of lithium along the a-axis and C-axis of BP. Reproduced with permission. ${ }^{27}$ Copyright 2012 , American Chemical Society. (c) The second galvanostatic charge/discharge profiles of the BP nanosheet, G paper, and BP-G hybrid paper electrodes at a current density of $100 \mathrm{~mA} \mathrm{~g}^{-1}$. Reproduced with permission. ${ }^{66}$ Copyright 2015, Wiley. (d) Schematics of in situ TEM setup of the half-cell battery. Reproduced with permission. ${ }^{67}$ Copyright 2019, Wiley. (e) Cycle behaviors and rate performance of the van der Waals heterostructures. Reproduced with permission. ${ }^{68}$ Copyright 2016, Elsevier. (f) Schematic illustration of the possible diffusion and transfer paths of $\mathrm{Li}^{+}$ions in the BPNs@TiO $\mathrm{BG}_{\mathrm{a}}$ composite electrode. Reproduced with permission. ${ }^{69}$ Copyright 2019, Elsevier. (g) Schematic of the crystal structure of BP (left) and lithium-intercalated BP (right). Reproduced with permission ${ }^{70}$ Copyright 2018, American Chemical Society. (h) Cycling stability curves of RP, BP-CP, and CP at a current density of 0.1C. Reproduced with permission. ${ }^{71}$ Copyright 2018, Elsevier.

the optimal space for Li ion intercalation is along either the $a$-axis or $c$-axis, as shown in Fig. 8b. In order to promote the scalable synthesis of high-quality bulk BP with high efficiency, Ren's group proposed the synthesis of centimeter-size bulk BP crystals through efficient mineralizer-assisted gas-phase transformation method and then fulfilled the scalable clean preparation of BP nanosheets by exfoliating the obtained BP crystals in water, taking advantage of its hydrophilic nature. ${ }^{66}$ After the fabrication of monolayer and few-layer BP sheets by mechanical exfoliation, satisfactory quality of the prepared BP was verified by the AFM test. Few-layered BP dispersion was firstly collected through mechanical grinding, tip sonication in water, and centrifugation for $30 \mathrm{~min}$, and then the BP-graphene hybrid (20 wt $\%$ graphene) flexible anode (BP-G) was prepared by tip-sonication (190 W, $60 \mathrm{~min})$ in NMP and vacuum filtration. As shown in Fig. 8c, apart from the great capacity enhancement from $200 \mathrm{~mA} \mathrm{~h} \mathrm{~g}{ }^{-1}$ (BP anode) to $930 \mathrm{~mA} \mathrm{~h} \mathrm{~g}^{-1}$ (BP-G hybrid flexible anode), two typical potential plateaus were identified at approximately 0.63 and $0.25 \mathrm{~V}$ for the BP-G hybrid paper electrode, which were related to the formation of $\mathrm{Li}_{x} \mathrm{P}$ and finally $\mathrm{Li}_{3} \mathrm{P}$ due to the enhancement of the electrical conductivity and reaction kinetics. On the other hand, only one short plateau for the BP anode at $0.25 \mathrm{~V}$ and a declined slope below $0.5 \mathrm{~V}$ for the bare graphene paper electrode were found., ${ }^{2,66}$ The exploration of
2D BP with high theoretical capacity opens a promising strategy for electrode research in lithium ion battery; thus, the intercalation mechanism of lithium should be fully understood. He's group used in situ TEM to investigate the electrochemically driven intercalation of lithium, as illustrated in Fig. 8d. The active BP electrode physically transferred on the TEM grid was connected with the movable Li counter electrode via a potentiostat to drive the electrochemical reactions within an in situ TEM holder. ${ }^{67}$ It indicates that lithium diffusion along the zigzag direction is energetically preferable, while diffusion along the armchair direction confronts an increased energy barrier, which might lead to an orientation-dependent intercalation channel. These findings provide insights into the fundamental mechanism of lithiation in BP.

Combing BP with a conductive matrix has been confirmed as a promising strategy to relieve the inherent disadvantages, i.e., low electronic conductivity and huge volume expansion. Ye et al. reported, for the first time, a facile strategy for the molecular level distribution of BP QDs on nitrogen-doped graphene, which prevents aggregation upon cycling and provides short lithium diffusion pathways for energy storage. ${ }^{68}$ Molecular level distribution was conducted in THF solution under facile stirring, during which the BP QDs were spontaneously assembled on the $\mathrm{N}$-graphene, taking advantage of the 
van der Waals interactions. Compared with N-graphene and BP QD anodes, the hybrid electrode gave an impressively high reversible capacity of $1271 \mathrm{~mA} \mathrm{~h} \mathrm{~g}{ }^{-1}$ after 100 cycles and excellent rate performance ( $470 \mathrm{~mA} \mathrm{~h} \mathrm{~g}{ }^{-1}$ even at $5000 \mathrm{~mA} \mathrm{~g}^{-1}$ ), emphasizing the synergistic superiority of the collected van der Waals heterostructures (Fig. 8e). In addition, another unique van der Waals heterostructure, BP nanosheet@TiO ${ }_{2} @ g r a p h e n e$ hydrogel, was explored by Sun's group. ${ }^{69} \mathrm{TiO}_{2}$ nanoparticles were firstly in situ grown on graphene networks (3D TiO $\left.{ }_{2} @ G\right)$ and then coated by the BP nanosheets, forming the final BPNs@TiO ${ }_{2} @ G$ heterostructure, which provided a widened potential window $(\sim 3.0 \mathrm{~V})$, increased initial capacity $\left(1336.1 \mathrm{~mA} \mathrm{~h} \mathrm{~g}^{-1}\right.$ at $\left.0.2 \mathrm{~A} \mathrm{~g}^{-1}\right)$, improved rate performance (271.1 $\mathrm{mA} \mathrm{h} \mathrm{g}^{-1}$ at $\left.5.0 \mathrm{~A} \mathrm{~g}^{-1}\right)$, and enhanced cycling stability (502 $\mathrm{mA} \mathrm{h} \mathrm{g}^{-1}$ after 180 cycles). The satisfactory electrochemical performance could be ascribed to the unique heterostructures combining BP nanosheet, $\mathrm{TiO}_{2}$, and graphene with improved electrical conductivity, enriched chemically-active defects, and rapid interlayer charge/mass transfer paths (Fig. 8f). ${ }^{69}$ Jia's group mechanically exfoliated BP nanosheets onto quartz substrates in the glovebox and then transferred them to a vacuum chamber for Li intercalation by evaporating the $\mathrm{Li}$ atoms. After heating treatment in hydrogen atmosphere, the intercalated lithium was transformed into lithium hydride ( $\mathrm{LiH}$ ) in the BP (Fig. 8g), showing better stability compared to the Li-intercalated sample or the pristine BP sample. ${ }^{70}$ In order to further avoid the large capacity drop after the first cycle and enhance the cycle performance, probably caused by the poor electrical conductivity, Tang and his coworkers prepared a kind of carbon paper with BP sheets grown on the surface by efficient thermal-vaporization of low-cost abundant red phosphorus in the argon-filled tube furnace. ${ }^{71}$ When applied in a two-electrode coin cell, the as-prepared BP on the conductive carbon paper (BP-CP) illustrated high initial capacity

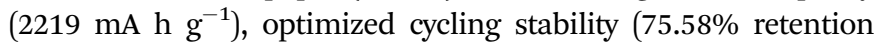
after 200 cycles), and excellent rate performance (Fig. 8h). The detailed comparison of the lithium storage performance of the typical BP-based electrodes is provided in Table 1.
4.1.2 Sodium ion battery. Though BP demonstrates excellent application in lithium-ion batteries, the increasing shortages and uneven distribution of lithium resources have raised increasing concerns on our heavy reliance on $\mathrm{Li}$ and further inspire researchers to explore BP's application in other energy storage devices. Owing to its abundance and similar electrochemical property to lithium, sodium ion batteries based on the BP electrode have been paid intensive attention. Haghighat-Shishavan et al. reported a kind of unique BP and multiwall carbon nanotubes composite, denoted as BP-CNT. The composite was obtained through a surface oxidationassisted chemical bonding procedure, in which controlled air exposure successfully induced the modification of BP with desired hydrophilicity, further facilitating stable bond formation on the functionalized CNTs. ${ }^{80}$ Taking advantage of the sufficient energy provided by the extensive mechanical deformation during ball milling, amorphous red phosphorus was firstly transformed into BP nanocrystals, and then mixed with carbon nanotubes and further milled under argon protection, forming the crosslinked BP-CNT composite electrode (Fig. 9a). When tested in sodium ion battery (SIB), the BP-CNT electrode showed a high initial Coulombic efficiency (ICE) (87.7\%) and impressive cycling stability (75.3\% retention after 200 cycles), providing a preferential way for the optimization of ICE and electrode integrity. To further improve the stability of BP during long cycling in SIB, Wang et al. bridged covalently-functionalized BP on graphene with the surface energy effectively reduced. Typically, the BP nanosheet linked to 4-nitrobenzenediazonium (4-NBD) through the $\mathrm{P}-\mathrm{C}$ bond was solvothermally reacted with graphene oxide, resulting in a reduced graphene/BP composite anode. The composite delivered excellent sodium storage capacity (1472 $\mathrm{mA} \mathrm{h} \mathrm{g}^{-1}$ at $0.1 \mathrm{~A} \mathrm{~g}^{-1}$ ) and good cycling stability (650 $\mathrm{mA} \mathrm{h} \mathrm{g}^{-1}$ at $1 \mathrm{~A} \mathrm{~g}^{-1}$ for the 200th cycle) with obviously swelling, as shown in the TEM images, due to the irreversible generation of polycrystalline $\mathrm{Na}_{3} \mathrm{P}$ during the discharge/charge process (Fig. 9b). ${ }^{81}$ Although combining BP with carbonaceous matrix is a common strategy to enhance the sodium storage

Table 1 Summary of lithium storage properties of BP-based active materials

\begin{tabular}{|c|c|c|c|c|c|c|}
\hline Electrode material & ICE & Capacity & Current & Cut voltage & Retention & Ref. \\
\hline BP-super P & $90.2 \%$ & $600 \mathrm{~mA} \mathrm{~h} \mathrm{~cm}^{-3}$ & $100 \mathrm{~mA} \mathrm{~g}^{-1}$ & $0.78-2.0 \mathrm{~V}$ & ca. $100 \% / 100$ cycles & 2 \\
\hline $\mathrm{BP} / \mathrm{NiCo} \mathrm{MOF}$ & $60.9 \%$ & $1215 \mathrm{~mA} \mathrm{~h} \mathrm{~g}^{-1}$ & $100 \mathrm{~mA} \mathrm{~g}^{-1}$ & $0.02-3.0 \mathrm{~V}$ & ca. $79 \% / 250$ cycles & 65 \\
\hline Red phosphorus induced BP & $53.8 \%$ & $453 \mathrm{~mA} \mathrm{~h} \mathrm{~g}^{-1}$ & $2 \mathrm{C}$ & $0-2.0 \mathrm{~V}$ & $50 \% / 60$ cycles & 27 \\
\hline BP-G hybrid paper & - & $501 \mathrm{~mA} \mathrm{~h} \mathrm{~g}^{-1}$ & $500 \mathrm{~mA} \mathrm{~g}^{-1}$ & $0.001-3 \mathrm{~V}$ & $80.2 \% / 500$ cycles & 66 \\
\hline BPQDs on N-graphene & $76.8 \%$ & $1271 \mathrm{~mA} \mathrm{~h}^{-1}$ & $500 \mathrm{~mA} \mathrm{~g}^{-1}$ & $0.01-2.0 \mathrm{~V}$ & $63.4 \% / 100$ cycles & 68 \\
\hline BPNs@TiO & $47.7 \%$ & $271.1 \mathrm{~mA} \mathrm{~h} \mathrm{~g}^{-1}$ & $5.0 \mathrm{~A} \mathrm{~g}^{-1}$ & $0.01-3.0 \mathrm{~V}$ & ca. $143.4 \% / 180$ cycles & 69 \\
\hline $\mathrm{BP}$-carbon paper & $97.7 \%$ & $2219 \mathrm{~mA} \mathrm{~h} \mathrm{~g}^{-1}$ & $0.1 \mathrm{C}^{\circ}$ & $0-2.0 \mathrm{~V}$ & $75.6 \% / 200$ cycles & 71 \\
\hline MWCNT@f-RP@BP hybrids & ca. $57.4 \%$ & $560 \mathrm{~mA} \mathrm{~h} \mathrm{~g}^{-1}$ & $500 \mathrm{~mA} \mathrm{~g}^{-1}$ & $0.01-3.0 \mathrm{~V}$ & ca. $74.7 \% / 250$ cycles & 74 \\
\hline BP@CNTs & $90 \%$ & $2004 \mathrm{~mA} \mathrm{~h}^{-1}$ & $100 \mathrm{~mA} \mathrm{~g}^{-1}$ & $0.01-2.0 \mathrm{~V}$ & $37.5 \% / 100$ cycles & 56 \\
\hline $\mathrm{BP}-\mathrm{TiO}_{2}-\mathrm{C}$ & $84.2 \%$ & $935.8 \mathrm{~mA} \mathrm{~h} \mathrm{~g}^{-1}$ & $2 \mathrm{Ag} \mathrm{g}^{-1}$ & $0.01-2.00 \mathrm{~V}$ & $85.3 \% / 300$ cycles & 60 \\
\hline P-Doped graphene & $74.0 \%$ & $1600 \mathrm{~mA} \mathrm{~h} \mathrm{~g}^{-1}$ & $10 \mathrm{~mA} \mathrm{~g}^{-1}$ & $0.01-3.0 \mathrm{~V}$ & $64 \% / 1700$ cycles & 58 \\
\hline $\mathrm{P}-\mathrm{KB}$ & $76.4 \%$ & $1449 \mathrm{~mA} \mathrm{~h} \mathrm{~g}^{-1}$ & $100 \mathrm{~mA} \mathrm{~g}^{-1}$ & $0.01-2.0 \mathrm{~V}$ & $90 \% / 100$ cycles & 59 \\
\hline NC@BP & $57.6 \%$ & $1020 \mathrm{~mA} \mathrm{~h} \mathrm{~g}^{-1}$ & $100 \mathrm{~mA} \mathrm{~g}^{-1}$ & $0.01-3.0 \mathrm{~V}$ & $87.1 \% / 230$ cycles & 75 \\
\hline BP/Ketjenblack-MWCNTs & $91 \%$ & $1600 \mathrm{~mA} \mathrm{~h} \mathrm{~g}^{-1}$ & $6.2 \mathrm{~A} \mathrm{~g}^{-1}$ & $0.02-2.0 \mathrm{~V}$ & $81 \% / 100$ cycles & 76 \\
\hline Few-layer BP & $78 \%$ & $2331 \mathrm{~mA} \mathrm{~h} \mathrm{~g}^{-1}$ & $500 \mathrm{~mA} \mathrm{~g}^{-1}$ & $0.01-2.0 \mathrm{~V}$ & $65 \% / 250$ cycles & 77 \\
\hline BPQD/TNS & $42 \%$ & $828 \mathrm{~mA} \mathrm{~h} \mathrm{~g}^{-1}$ & $100 \mathrm{~mA} \mathrm{~g}^{-1}$ & $0.005-3.0 \mathrm{~V}$ & $77.6 \% / 2400$ cycles & 78 \\
\hline$(\mathrm{BP}-\mathrm{G}) / \mathrm{PANI}$ & $76 \%$ & $1650 \mathrm{~mA} \mathrm{~h}^{-1}$ & $260 \mathrm{~mA} \mathrm{~g}^{-1}$ & $0.001-2.5 \mathrm{~V}$ & $79.1 \% / 2000$ cycles & 79 \\
\hline BP@irGO & $81.4 \%$ & $1515 \mathrm{~mA} \mathrm{~h} \mathrm{\textrm {g } ^ { - 1 }}$ & $260 \mathrm{~mA} \mathrm{~g}^{-1}$ & $0.01-2.5 \mathrm{~V}$ & ca. $75 \% / 100$ cycles & 18 \\
\hline
\end{tabular}


a

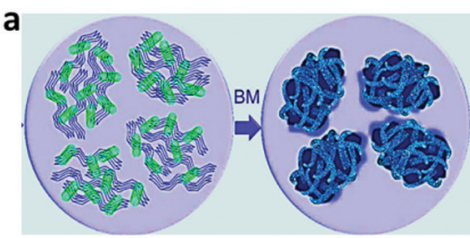

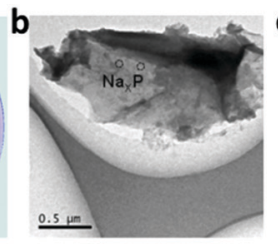

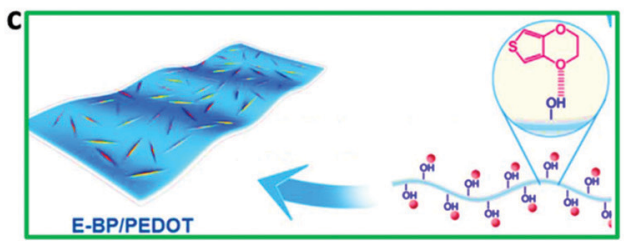

d
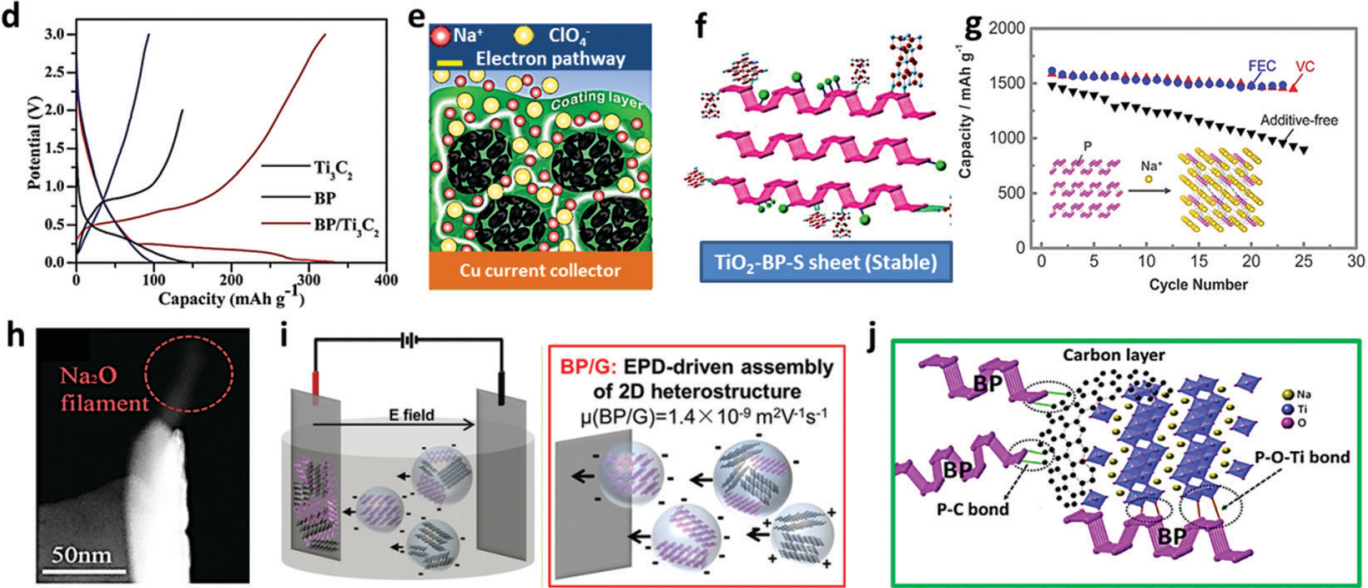

Fig. 9 Application of BP in sodium ion batteries. (a) Schematic achievement of the BP-CNT composite for the SIB anode. Reproduced with permission. ${ }^{80}$ Copyright 2018, Royal Society of Chemistry. (b) Typical TEM image of 4-NBD modified BP after 10 electrochemical cycles. Reproduced with permission. ${ }^{81}$ Copyright 2017, American Chemical Society. (c) In situ growth of PEDOT fibers on the surface-modified BP nanosheets at room temperature. Reproduced with permission. ${ }^{82}$ Copyright 2017, Elsevier. (d) Charge-discharge profiles of the $\mathrm{BP}, \mathrm{Ti}_{3} \mathrm{C}_{2}$, and $\mathrm{BP} / \mathrm{Ti}_{3} \mathrm{C}_{2}$ composite electrodes. Reproduced with permission. ${ }^{83}$ Copyright 2019, Royal Society of Chemistry. (e) Schematic diagram of the sodiation of BP-G/PANI electrodes. Reproduced with permission. ${ }^{84}$ Copyright 2019, American Chemical Society. (f) Schematic illustration of the stable TiO ${ }_{2}-\mathrm{BP}-\mathrm{S}$ nanosheets. Reproduced with permission. ${ }^{85}$ Copyright 2019, Wiley. (g) BP electrode in SIBs with or without additives. Reproduced with permission. ${ }^{28}$ Copyright 2016 , American Chemical Society. (h) In situ TEM image of the BP nanosheet after partial sodiation. Reproduced with permission. ${ }^{86}$ Copyright 2020 , Royal Society of Chemistry. (i) Schematic illustration of the EPD process to assemble the 2D BP/G heterostructures. Reproduced with permission. ${ }^{87}$ Copyright 2018, Royal Society of Chemistry. (j) Schematic illustration of the NTO/C-BP hybrids. Reproduced with permission ${ }^{88}$ Copyright 2018 , American Chemical Society.

stability by improving the reaction kinetics and mitigating the large volume change during de/sodiation, it typically leads to reduced specific capacity based on the overall mass of the whole electrode. Thus, Dou's group reported the nanoscale surface engineering of exfoliated surface-modified BP through the homogeneous deposition of horizontally aligned poly(3,4ethylenedioxythiophene) (PEDOT) nanofibers (Fig. 9c), which could ensure a continuous conductive network as well as super-wettability with the electrolyte and further enhance the sodium storage capacity from $345 \mathrm{~mA} \mathrm{~h} \mathrm{~g}^{-1}$ (few-layered BP) to $1597 \mathrm{~mA} \mathrm{~h} \mathrm{~g}{ }^{-1}$ (BP/PEDOT) at $100 \mathrm{~mA} \mathrm{~g}{ }^{-182} \mathrm{Ti}_{3} \mathrm{C}_{2}$ with controllable morphology, has been widely studied as an anode material for sodium-ion batteries, and can be composited with other active materials with high theoretical specific capacity. $\mathrm{Ma}$ and his co-workers rationally designed a $\mathrm{BP} / \mathrm{Ti}_{3} \mathrm{C}_{2}$ MXene composite combining small BP nanoparticles with exfoliated $\mathrm{Ti}_{3} \mathrm{C}_{2}$ layers. The BP powder originated from red phosphorus was ball-milled in DMF. After sonication and centrifugation, the BP suspension was collected and further mixed with $\mathrm{Ti}_{3} \mathrm{C}_{2}$ nanoflakes obtained by selectively etching $\mathrm{Al}$ from $\mathrm{Ti}_{3} \mathrm{AlC}_{2}$. The $\mathrm{BP} / \mathrm{Ti}_{3} \mathrm{C}_{2}$ composite was finally collected by facile filtration, which exhibited an improved discharge specific capacity of $331.9 \mathrm{~mA} \mathrm{~h} \mathrm{~g}{ }^{-1}$ compared to $\mathrm{BP}$ and $\mathrm{Ti}_{3} \mathrm{C}_{2}$ electrodes (146.1 and $98.5 \mathrm{~mA} \mathrm{~h} \mathrm{~g}^{-1}$, respectively) (Fig. 9d). ${ }^{83}$ Wan et al. reported a ternary composite comprising of $\mathrm{BP}$, graphite, and polyaniline (BP-G/PANI) with a BP mass content of $c a$. $65 \mathrm{wt} \%$, in which PANI offered an optimized Na-ion pathway (electrolyte $\rightarrow$ PANI $\rightarrow$ $\mathrm{BP}-\mathrm{G} \rightarrow \mathrm{BP}$ ) between the electrolyte and active material (BP-G), while graphite in the electrode could further reduce the ion charge transfer resistance, as shown in Fig. 9e. ${ }^{84}$ Taking advantage of the rationally designed ternary composite, the BP-G/PANI anode provided an ultrahigh reversible specific capacity of $1530 \mathrm{~mA} \mathrm{~h} \mathrm{~g}^{-1}$ at $0.25 \mathrm{~A} \mathrm{~g}^{-1}$, which still remained $520 \mathrm{~mA} \mathrm{~h} \mathrm{~g}^{-1}$ even after 1000 cycles at $4 \mathrm{~A} \mathrm{~g}^{-1}$ with an extremely low capacity fading rate $(0.024 \%)$. This method paves a new way for the practical design of the BP-based electrode with synergistic effect between the high theoretical active material and reliable carbon/polymer matrix. As we all know, bare BP without suitable modification is not suitable for battery application due to its rapid capacity decrease and high instability in air atmosphere. Aiming to address the mentioned issues, Xia et al. prepared sulfur-doped $\mathrm{BP}^{-\mathrm{TiO}_{2}}$ hybrids $\left(\mathrm{TiO}_{2}-\mathrm{BP}-\mathrm{S}\right)$ (Fig. 9f) with $\mathrm{P}-\mathrm{S}$ and $\mathrm{P}-\mathrm{Ti}$ bonds formed during one-step high-energy ball milling of $0.2 \mathrm{~g}$ of BP, $0.8 \mathrm{~g}$ of $\mathrm{TiO}_{2}$, and $0.06 \mathrm{~g}$ of sulfur in a $50 \mathrm{~mL}$ stainless steel vessel with a rotation rate of $750 \mathrm{rpm}$ for $24 \mathrm{~h}$. The hybrids delivered good stability in air, desirable sodium storage capacity (490 $\mathrm{mA} \mathrm{h} \mathrm{g}^{-1}$ after 100 cycles at $50 \mathrm{~mA} \mathrm{~g}^{-1}$ ), and impressive stability (96\% capacity retention after 300 cycles). ${ }^{85}$ With the 
help of XPS analysis, the effect of sulfur doping on the stability of BP was further evaluated. After exposure to the same ambient conditions for $45 \mathrm{~d}\left(25{ }^{\circ} \mathrm{C}, \mathrm{RH}=30 \%, \mathrm{pH}=7\right), 85.2 \%$ of phosphorus in the $\mathrm{TiO}_{2}-\mathrm{BP}$ sample was oxidized, while only $20.5 \%$ of phosphorus in the fresh $\mathrm{TiO}_{2}-\mathrm{BP}-\mathrm{S}$ sample was oxidized due to the presence of $\mathrm{P}-\mathrm{S}$ bonds during the ball-milling process. $^{85}$ In order to have a deep understanding of the electrode/electrolyte interface of the BP based electrodes, which can significantly influence its electrochemical performance during cycling, the surface of the BP anode was systematically investigated by Komaba et al. using powerful surface characterization techniques. BP obtained by a high-pressure method was ball milled together with acetylene black and then applied in the sodium half cells. The electrolyte solution without or with additives (5 vol\% FEC and 1 vol\% VC) further revealed the influence of the additives on the SEI formation. The SEI surface layers modified by FEC and VC could induce a stable surface layer and protect the electrolytes against decomposition, leading to a high sodium storage capacity $\left(1600 \mathrm{~mA} \mathrm{~h} \mathrm{~g}{ }^{-1}\right)$ and good reversibility (91\% retention after 20 cycles) of the desodiation/ sodiation of the black P electrode (Fig. 9g). ${ }^{28}$ Using in situ TEM, Zhang et al. identified the anisotropic sodium diffusion during the sodiation process and the formation of alternating layered and amorphous lamella in the BP nanosheets with small volume expansion was induced by partial sodiation (Fig. 9h). They further explored the cycling performance of BP with controlled capacities in half cell SIBs, aiming to reach improved anode stability by suppressing the sodiation depth to some extent and preserving the layered structure of BP. ${ }^{86}$ It shows that when the discharge capacity was set to $400 \mathrm{~mA} \mathrm{~h} \mathrm{~g}^{-1}$, the SIB could maintain a long cycling (above 100 cycles) with a high active material loading level ( $c a .2 \mathrm{mg} \mathrm{cm}^{-2}$ ), much longer than those with the capacity set to $600 \mathrm{~mA} \mathrm{~h} \mathrm{~g}^{-1}$ or above, which sheds light on the effectiveness of the partial sodiation method for the enhancement of BP-based SIB. ${ }^{86}$ Binder-free BP/graphene anode was delicately prepared by Pint et al. by the co-exfoliation of $2 \mathrm{D}$ graphene and BP materials, forming 2D heterostructured material in NMP solvents owing to the preferable electrostatic surface interaction between the BP sheet and graphene, which was then assembled on conductive stainless steel using electrophoretic deposition (EPD) (Fig. 9i). ${ }^{87}$ The stainless steel electrode after EPD without adding a binder or conductive carbon additive could provide extraordinary sodium storage capacity (2365 $\mathrm{mA} \mathrm{h} \mathrm{g}^{-1}$ at $100 \mathrm{~mA} \mathrm{~g}^{-1}$ ), giving an insight into the strategies for scalable and facile assembling of complex heterostructured 2D materials with utilization of high energy-density sodium ion batteries. A novel and stable sodium titanate/ carbon-BP (NTO/C-BP) hybrid was fabricated by Xia and his co-workers for the first time through the mechanical milling of the mixture of NTO/C and BP (700 rpm for $12 \mathrm{~h}$ ) under argon atmosphere as a promising anode material for advanced sodiumion batteries, showing high specific capacity $\left(225 \mathrm{~mA} \mathrm{~h} \mathrm{~g}{ }^{-1}\right.$ after 55 cycles at $\left.20 \mathrm{~mA} \mathrm{~g}^{-1}\right)$ and long cycling property $\left(143.3 \mathrm{~mA} \mathrm{~h} \mathrm{~g}^{-1}\right.$ after 400 cycles at $\left.200 \mathrm{~mA} \mathrm{~g}^{-1}\right){ }^{88}$ The NTO/C-BP hybrids collected by the mechanical milling of NTO/C from the simple sol-gel process and the $\mathrm{BP}$ nanoparticles from planetary ball-milling contained strong $\mathrm{P}-\mathrm{O}-\mathrm{Ti}$ bonds and stable $\mathrm{P}-\mathrm{C}$ bonds (Fig. 9j), which could remarkably mitigate the volume change during sodiation and optimize the electrode endurance.

4.1.3 Potassium ion batteries. Potassium ion battery (KIB) is an emerging alternative battery system owing to its comparable abundance of sodium, higher operating potential, and improved energy density, considering that the $\mathrm{K} / \mathrm{K}^{+}$couple offers a lower redox potential of $-2.92 \mathrm{~V}$ compared to that of sodium $(-2.71 \mathrm{~V})$. In addition, cheaper aluminum current collectors can be applied to hold the anode material in KIBs since no alloying occurs between potassium and aluminum even at a low potential. Taking the fairly light atomic quality and large Li-uptake and Na-uptake ability into account, BP can also be applied in KIBs with a high theoretical capacity of $2596 \mathrm{~mA} \mathrm{~h} \mathrm{~g}{ }^{-1}$ by forming $\mathrm{K}_{3} \mathrm{P} \cdot{ }^{98,99}$ Phosphorus has three allotropes (i.e., white phosphorus, red phosphorus, and black phosphorus). White phosphorus is toxic and chemically unstable. Although RP has abundant reserves and low cost, its conductivity is relatively low, while BP with high electron mobility is the physicochemically most stable one and can be applied as an electrode active material. ${ }^{100,101}$ Liu et al. investigated the surface adsorption and bulk penetration of $\mathrm{K}$ ions in $\mathrm{BP}$ and the $\mathrm{K}-\mathrm{P}$ reaction process in detail based on firstprinciples calculation (Fig. 10a), and estimated the potassium storage performance of the BP anode in $\mathrm{KIB}\left(864.8 \mathrm{~mA} \mathrm{~h} \mathrm{~g}{ }^{-1}\right)$, providing valuable information for the further fabrication of BP-based electrodes in the KIB system. Compared with lithium and sodium, potassium has the lowest energy barrier for diffusion in the bulk BP (0.182 eV in zigzag migration path and $2.013 \mathrm{eV}$ in armchair migration path). However, the formation of the $\mathrm{K}_{3} \mathrm{P}$ phase could be found based on the calculation of the binary phase alloy, different from the cases of $\mathrm{Li}-\mathrm{P}$ and Na-P. ${ }^{89}$ Although there is still an alloy-dealloy potassium storage process, only the KP (not $\mathrm{K}_{3} \mathrm{P}$ ) alloy could be identified in the final transformation, allowing enhanced electrode stability in KIBs. First principles calculation was also adopted by Li et al. to check the GeSe/BP heterostructure as a promising anode material for KIBs, revealing that GeSe/BP could experience a semiconductor-to-metal transition after the incorporation of potassium and maintain enhanced conductivity and lower energy barrier for potassium diffusion compared to monolayer GeSe. ${ }^{90}$ In addition, it showed that the GeSe/BP anode could accommodate five layers of potassium with strong ionic bonding (Fig. 10b), which significantly promoted the potassium storage capability, thus verifying the appealing potential for application as anodes in KIBs. Glushenkov and his co-workers prepared the $\mathrm{BP} /$ graphite composite through the mechanical milling of the mixture. The composite exhibited an initial specific capacity of $617 \mathrm{~mA} \mathrm{~h} \mathrm{~g}^{-1}$, more than two times higher than that of graphite in KIBs. ${ }^{91}$ Fig. 10c illustrates the cycling stability between the $\mathrm{BP} /$ graphite anode with two different ratios $(7: 3$ and $1: 1)$ at $50 \mathrm{~mA} \mathrm{~g}^{-1}$. The anode $(7: 3)$ suffers from a continuous decline in the capacity, maintaining a small specific capacity of $30 \mathrm{~mA} \mathrm{~h} \mathrm{~g}{ }^{-1}$ after 50 cycles, while the BP/graphite anode (1:1) has relatively stable cycling performance with $270 \mathrm{~mA} \mathrm{~h} \mathrm{~g}$ maintained after 50 cycles, corresponding to the good retention of $61 \%$. Besides, the result further emphasizes the feasibility of 

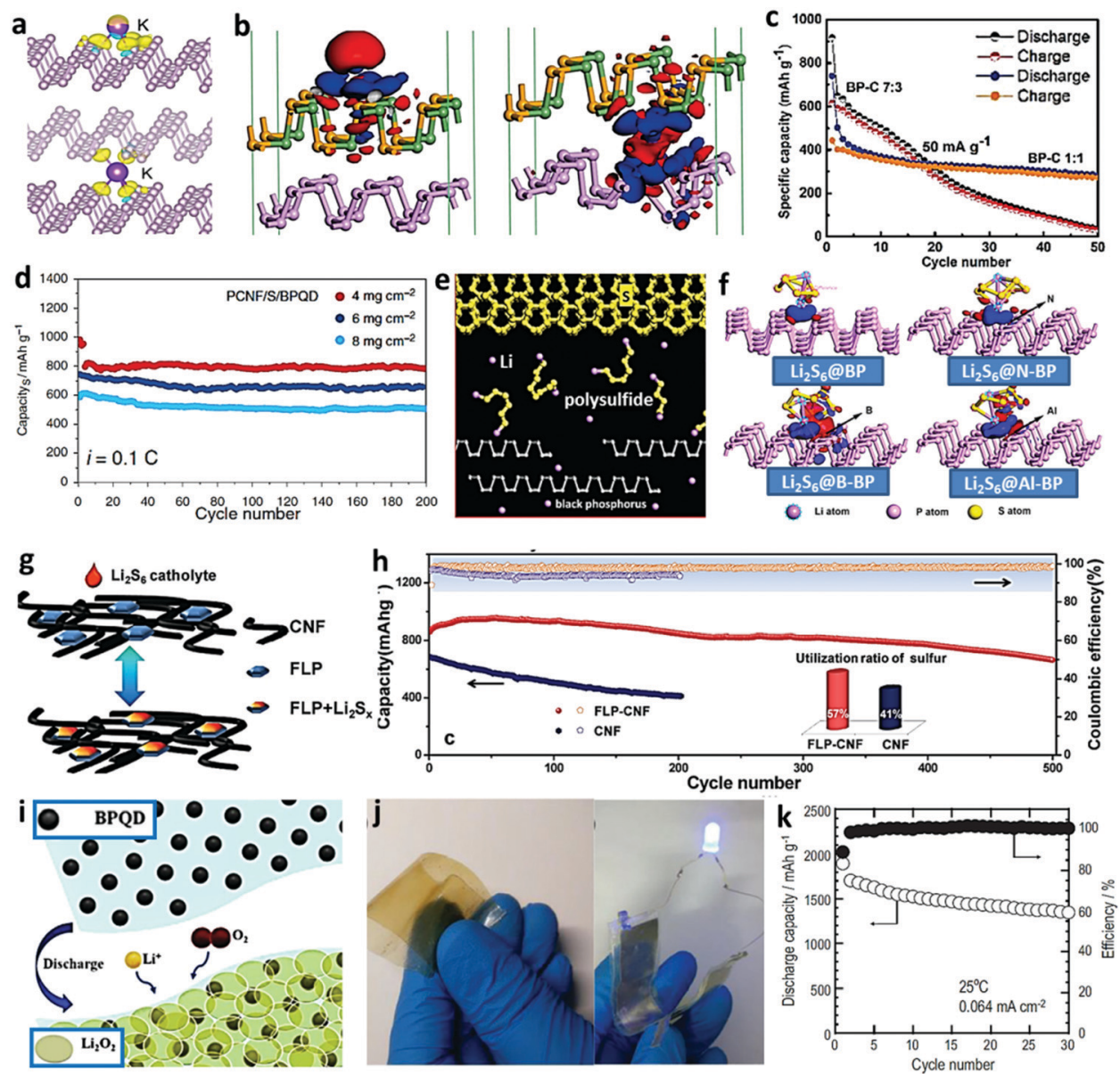

Fig. 10 Application of BP-based electrodes in potassium ion batteries (a-c), lithium sulfur batteries (d-h), lithium oxygen batteries (i), zinc-nickel batteries (j), and all-solid-state batteries ( $\mathrm{k}$ ). (a) Differential charge densities of the BP layers adsorbed (upper) and inserted (lower) with a $\mathrm{K}$ atom. Reproduced with permission. ${ }^{89}$ Copyright 2019, Springer. (b) Differential charge densities of $\mathrm{K}$ adsorbed on the surface (left) and in the interlayer (right) of GeSe/BP. Reproduced with permission. ${ }^{90}$ Copyright 2019, American Chemical Society. (c) Cycling stabilities of the BP-C composites with different ratios. Reproduced with permission ${ }^{91}$ Copyright 2017, Royal Society of Chemistry. (d) Cyclic performance of the PCNF/S/BPQD at 0.1C for 200 cycles with increasing sulfur loadings of 4,6 , and $8 \mathrm{mg} \mathrm{cm}^{-2}$. Reproduced with permission. ${ }^{92}$ Copyright 2018 , Nature. (e) Operating principle of the BP coating layer in the Li-S battery. Reproduced with permission. ${ }^{93}$ Copyright 2016, Wiley. (f) Comparison of the charge density difference of polysulfide $\mathrm{Li}_{2} \mathrm{~S}_{6}$ on the $\mathrm{BPs}$ surface. Reproduced with permission ${ }^{94}$ Copyright 2020, Elsevier. (g) Schematic of the FLP-CNF matrix used as the host for the lithium polysulphide catholyte and (h) its corresponding cycling performance at a current density of $1 \mathrm{C}$. Reproduced with permission. ${ }^{95}$ Copyright 2016 , Wiley. (i) Schematic demonstration of $\mathrm{Li}_{2} \mathrm{O}_{2}$ formation on $\mathrm{BPQD} / \delta-\mathrm{MnO}_{2}$. Reproduced with permission ${ }^{96}$ Copyright 2019, Elsevier. (j) Demonstration of the flexible zinc-nickel battery using the PVA@BP electrolyte membrane. Reproduced with permission. ${ }^{97}$ Copyright 2018, Elsevier. (k) Cycle performance of the all-solid-state cells using the composites with BP and acetylene black. Reproduced with permission. ${ }^{30}$ Copyright 2010, Elsevier.

the BP-based anodes with reliable potassium storage capacities, comparable with those of LIBs and SIBs.

4.1.4 Lithium sulfur batteries. During the pursuit of nextgeneration energy storage devices, lithium-sulfur batteries have several appealing advantages, such as low cost, light weight, and high energy density ${ }^{102}$ Nevertheless, a number of shortcomings, including intrinsic insulation, active material loss due to the formation of sulfide, and capacity fading as a result of volume expansion, still hinder their wide application. Thus, researchers have paid considerable attention to suitable strategies to solve the bottlenecks, among which adding additive agents (carbon black, carbon nanotubes, or graphene) have been explored for performance optimization. ${ }^{103,104}$ Lau et al. employed BP QDs as electrocatalysts to overcome the shuttling and sluggish conversion of polysulfides. With the help of numerous catalytically active sites on the edge of the BP QDs, the obtained porous carbon nanofiber/sulfur/BP QDS electrode (PCNG/S/BPQD) showed satisfactory reaction kinetics, suppressed the shuttling of polysulfides, and maintained a low capacity decreasing rate $(0.027 \%$ per cycle over 1000 cycles $) .^{92}$ In addition, when the electrode mass loading was increased to 4,6 , and $8 \mathrm{mg} \mathrm{cm}^{-2}$, the cyclic capacity retentions were still as high as $95 \%, 90 \%$, and $89 \%$ even after 200 cycles at a current density of $0.1 \mathrm{C}$, implying that the BP QDs could still effectively immobilize the flooded polysulfides in thick electrodes and further improve the performance of the lithium sulfur battery (Fig. 10d). Cui and his colleagues designed a modified separator membrane for the lithium-sulfur battery through the delicate deposition of BP nanoflakes onto a commercial polypropylene separator. The dispersion of thin BP nanoflakes 
was obtained by intensive sonication (Branson 5210 Ultrasonic) for $10 \mathrm{~h}$ and centrifugation for $30 \mathrm{~min}$ at $3000 \mathrm{rpm}$ to remove the sediment, then mixed with $8 \mathrm{wt} \%$ polyvinylidene fluoride (PVDF), and vacuum filtered by a Celgard polyethylene membrane, forming the BP-modified separator with a uniform and dense BP layer $\left(0.4 \mathrm{mg} \mathrm{cm}^{-2}\right)$, covering the nanopores. ${ }^{93}$ As illustrated in Fig. 10e, the BP-modified side, facing the sulfur-based cathode electrode, could effectively intercept and bind the polysulfides and prevent their penetration through the polypropylene membrane, leading to an significantly enhanced cycling performance ( $800 \mathrm{~mA} \mathrm{~h} \mathrm{~g}^{-1}$ after 100 cycles) with a retention rate of $86 \%$, much higher than those of electrodes with a graphene-modified separator and a conventional separator ( $66 \%$ and $8 \%$, respectively). This research opens new avenues to improve the performance of lithium-sulfur batteries with rational $\mathrm{BP}$ modification.

Heteroatom doping is an important way to improve the surface performance by regulating the surface polarity and elevating the active sites for transformation. ${ }^{105}$ In order to predict the effect of the "surface strategy" on the enhancement of the BP-based anode in the lithium-sulfur battery, $\mathrm{Wu}$ and co-workers performed theoretical DFT calculations with Lewis acidic B-doped BP, Al-doped BP, Lewis basic N-doped BP, and pristine $\mathrm{BP}$ as objects. The result revealed that the order of adsorption energy is B-doped BP $\geq$ Al-doped BP $>$ N-doped $\mathrm{BP}>$ pristine BP. N-Doping increased the energy required for lithium polysulfide conversion, whereas B-doping and Al-doping reduced the energy requirement and further enhanced the performance of the BP-based electrode for lithium sulfur battery (Fig. 10f). ${ }^{94}$ Koratkar's group designed a few-layered $\mathrm{BP} /$ carbon nanofiber (FLP/CNT) composite applied in the $\mathrm{Li}-\mathrm{S}$ battery through the vacuum filtration of the suspension in NMP containing commercial carbon nanofiber and few-layer BP prepared under tip sonication of the bulk BP powder (Fig. 10g). ${ }^{95}$ The as-collected FLP-CNF film was then utilized as the host for dissolved lithium polysulphides, the active materials that react with lithium, providing an enhanced long-term cycling performance (660 $\mathrm{mA} \mathrm{h} \mathrm{g}^{-1}$ after 500 cycles at 1C) with an average capacity decay per cycle of only $0.053 \%$, which is much lower than that of the pure CNF electrode $(0.25 \%)$ due to the significant promotion of few-layer BP as an electrocatalyst and a polysulfide immobilizer (Fig. 10h).

4.1.5 Lithium oxygen batteries. Recently, lithium-oxygen $\left(\mathrm{Li}-\mathrm{O}_{2}\right)$ cells have attained increasing attention due to their extremely high theoretical energy density of $3505 \mathrm{~W} \mathrm{~h} \mathrm{~kg}{ }^{-1}$, which is far beyond that of current LIBs. ${ }^{106,107}$ Zhao et al. proposed a novel fabrication method of catalytic cathode comprising of $\mathrm{BP}$ QDs decorated $\delta-\mathrm{MnO}_{2}$ nanobelts (BPQD/ $\delta-\mathrm{MnO}_{2}$ ) on carbon cloth (Fig. 10i), which could provide abundant catalytic sites, facilitate barrier-free transportation of the reactants, and result in the conformal growth of thin $\mathrm{Li}_{2} \mathrm{O}_{2}$ sheets on $\delta-\mathrm{MnO}_{2}$. The cell showed an excellent specific capacity of $8463 \mathrm{~mA} \mathrm{~h} \mathrm{~g}^{-1}$ at $100 \mathrm{~mA} \mathrm{~g}^{-1}$ and impressive cycling stability (182 cycles at $\left.400 \mathrm{~mA} \mathrm{~g}^{-1}\right) .{ }^{96}$ It is noteworthy BPQD/ $\delta-\mathrm{MnO}_{2}$ was obtained in a facile way, in which the $\delta-\mathrm{MnO}_{2} /$ carbon cloth was immersed in the BPQD yellow supernatant before fast centrifugation, which could serve as a valuable acceleration of BP utilization in the lithium-oxygen battery.

4.1.6 Zinc-nickel batteries. In order to avoid the inflammation of the organic electrolyte in the lithium-ion battery, rechargeable and low-cost zinc-nickel battery with environmental-friendliness was proposed with the nonflammable aqueous electrolytes used. ${ }^{108}$ Flexible zinc-nickel battery with BP-modified polyvinyl acetate gel-polymer (PVA@BP) electrolyte was designed by Li et al., where PVA acted as the polymer matrix and $\mathrm{BP}$ as the $\mathrm{Zn}(\mathrm{OH})_{4}{ }^{2-}$ barrier. $^{97}$ The BP modification of PVA with high flexibility was applied in the electrode assemble, which showed a voltage of $1.712 \mathrm{~V}$ and could light up an LED bulb even in the bent state when the cell was fully charged (Fig. 10j). The Tafel test revealed that BP addition in the electrolyte could effectively hinder the corrosion of zinc, inhibit the hydrogen evolution reaction, and optimize the coulombic efficiency, leading to excellent initial discharge capacity (509.8 $\mathrm{mA} \mathrm{h} \mathrm{g}^{-1}$ ) and stable cycling for 100 cycles. $^{97}$

4.1.7 All-solid-state batteries. All-solid-state lithium secondary batteries using inorganic solid electrolytes have attracted much attention from the point-of-view of their safety and high energy density. ${ }^{109}$ Tatsumisago et al. synthesized BP by the mechanical milling technique using red phosphorus powder as the starting material. The collected BP was further milled with acetylene black, mixed with the $80 \mathrm{Li}_{2} \mathrm{~S} \cdot 20 \mathrm{P}_{2} \mathrm{~S}_{5}$ solid electrolyte, and applied to the working electrode. ${ }^{30}$ Although the all-solid-state battery with red phosphorus active material illustrated an initial discharge capacity of $1345 \mathrm{~mA} \mathrm{~h} \mathrm{~g}^{-1}$, there is an ultralow Coulombic efficiency of $12 \%$. The cell with the BP electrode provided a discharge capacity of $1777 \mathrm{~mA} \mathrm{~h} \mathrm{~g}^{-1}$ and enhanced Coulombic efficiency (61\%). The milled BP/acetylene black composite showed the highest specific capacity (1962 mA h g ${ }^{-1}$ ) and Coulombic efficiency (89\%). When the all-solid-state cells with the milled BP/acetylene black composites were cycled at $0.064 \mathrm{~mA} \mathrm{~cm}^{-2}$, it exhibited a capacity of $1350 \mathrm{~mA} \mathrm{~h} \mathrm{~g}{ }^{-1}$ for 30 cycles with little capacity fading (Fig. 10k), further indicating the great potential for BP application in all-solidstate lithium rechargeable batteries with sulfide electrolytes. ${ }^{30}$

\subsection{Supercapacitors}

As an energy storage device with excellent cycling performance, high energy density, and fast charging/discharging rate, supercapacitors have attracted extensive attention. Nevertheless, achieving high energy density via practical materials and synthetic techniques is a major challenge. Thus, Chen et al. prepared a heterostructured BP/CNT composite, in which carbon nanotubes were chemically connected with BP nanosheets through the $\mathrm{P}-\mathrm{C}$ bond under a high-temperature treatment. The as-obtained BP/CNT was then assembled into a non-woven fiber fabric by the microfluidic-spinning technique, followed by the hot-pressing of two conductive fabric layers and one polymer-supported ionic liquid electrolyte layer into the supercapacitors to power electronics, including LEDs, a smart watch, and displays (Fig. 11a). ${ }^{110}$ In addition, the rationally designed supercapacitor was produced with enhanced mechanical stability, alleviated layer restacking of the BP nanoflake, and ionic channels for fast ion diffusion, thus providing high energy 
a
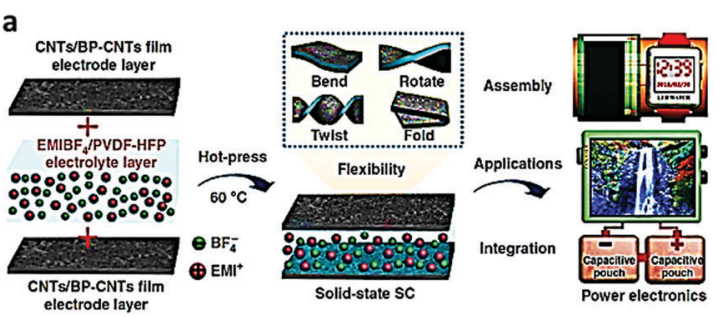

$b_{\text {BP/PANI nanocomposite }}$
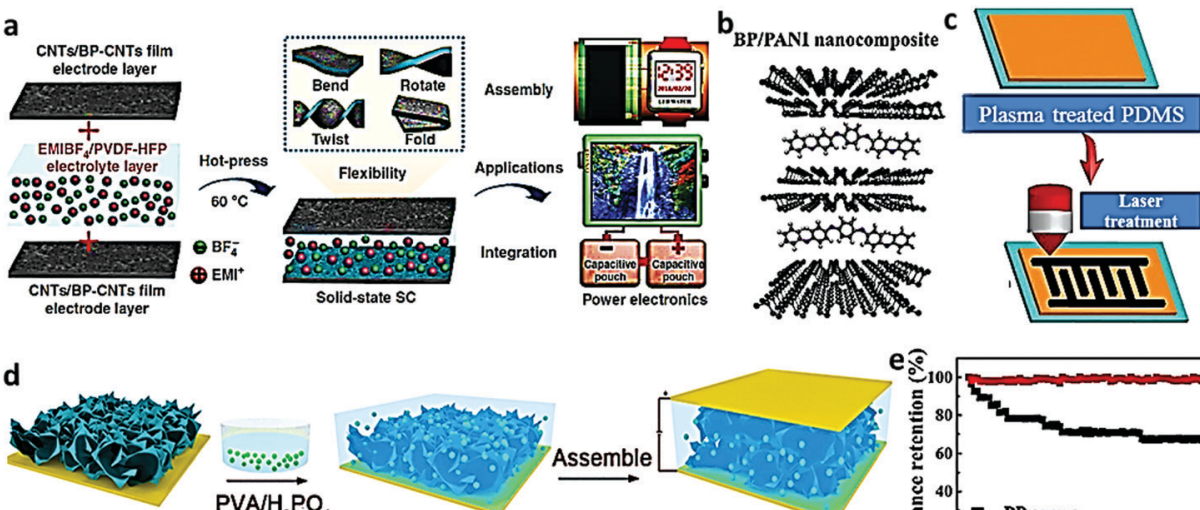

PET/Au/BP

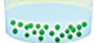

$\overrightarrow{\mathrm{PVANH}_{3} \mathrm{PO}}$
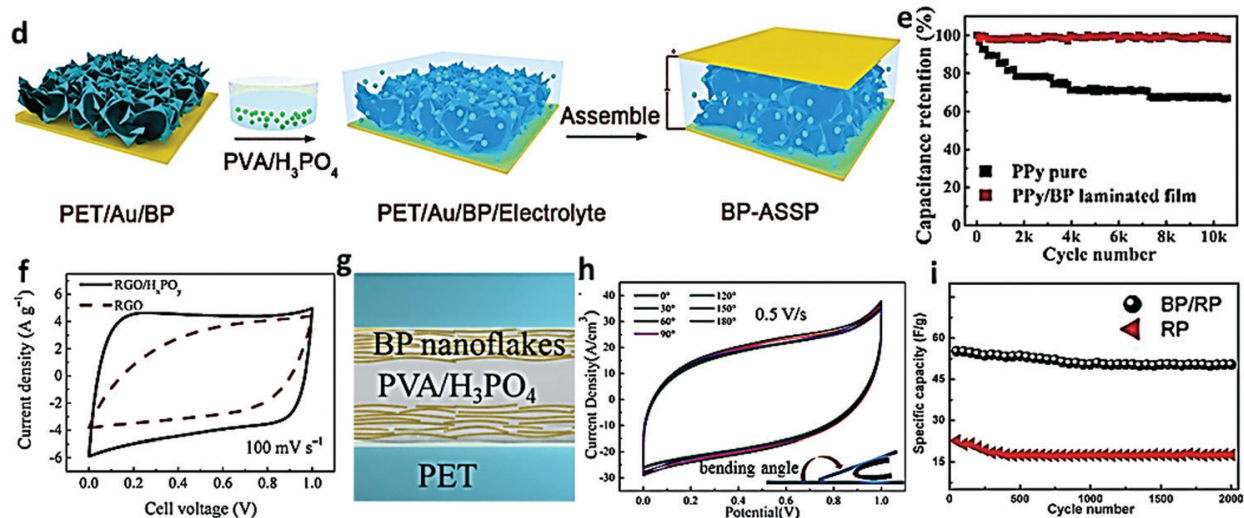

PET/Au/BP/Electrolyte

BP-ASSP
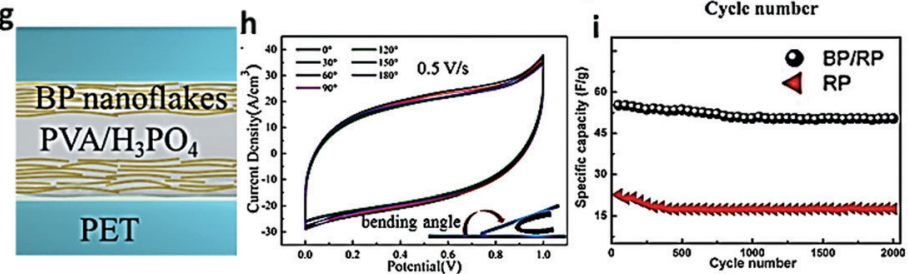

Fig. 11 Various BP based supercapacitors. (a) Construction of flexible supercapacitor by hot pressing of two conductive fabric layers and one polymersupported ionic liquid electrolyte layer. Reproduced with permission. ${ }^{110}$ Copyright 2018, Nature. (b) Fabrication of BP/PANI Nanocomposite. Reproduced with permission. ${ }^{111}$ Copyright 2017, American Chemical Society. (c) Schematic illustration of DLW fabrication of flexible planar supercapacitors based on GO and BPQD composites. Reproduced with permission. ${ }^{112}$ Copyright 2019, Royal Society of Chemistry. (d) Schematic illustration of the fabrication of BP-ASSP. Reproduced with permission. ${ }^{113}$ Copyright 2019, Royal Society of Chemistry. (e) Capacitance retention of PPy/BP laminated film and pure PPy film after 10000 cycles under a current density of $5 \mathrm{~A} \mathrm{~g}^{-1}$. Reproduced with permission. ${ }^{114}$ Copyright 2018, American Chemical Society. (f) Comparison of cyclic voltammetry curves of supercapacitors based on $\mathrm{RGO} / \mathrm{H}_{x} \mathrm{PO}_{y}$ and washed RGO films at $100 \mathrm{mV} \mathrm{s}^{-1}$. Reproduced with permission. ${ }^{170}$ Copyright 2018, American Chemical Society. (g) Cross section of the sandwich structure of BP based ASSP devices. Reproduced with permission. ${ }^{115}$ Copyright 2016 , Wiley. (h) CV curves of BP/CNT electrode at a scan rate of $0.5 \mathrm{~V} \mathrm{~s}^{-1}$ with differently bent configurations. Reproduced with permission. ${ }^{116}$ Copyright 2017 , American Chemical Society. (i) Capacity retention of RP and BP/RP after 2000 cycles at $1 \mathrm{~A} \mathrm{~g}^{-1}$. Reproduced with permission. ${ }^{117}$ Copyright 2017 , Royal Society of Chemistry.

density (96.5 $\left.\mathrm{mW} \mathrm{h} \mathrm{cm}^{-3}\right)$, large capacitance $\left(308.7 \mathrm{~F} \mathrm{~cm}^{-3}\right)$, long-life cycling stability (90.2\% retention after 10000 cycles), and durability upon deformation (Young's modulus: $313 \mathrm{MPa}$; break elongation: 17.96\%). This research gives a helpful guidance for the development of BP-based flexible and wearable supercapacitors. Pumera's group demonstrated a unique hybrid electrode material composed of few-layered BP and polyaniline (PANI). BP nanosheets prepared by high pressure treatment, sonication, and ball milling was mixed with aniline monomer before the rapid addition of ammonium persulfate (APS), triggering the start of in situ polymerization. ${ }^{111}$ After centrifugation and vacuum drying, the BP/PANI nanocomposites were collected (Fig. 11b), demonstrating high capacitance $\left(354 \mathrm{~F} \mathrm{~g}^{-1}\right)$ at a discharge current of $0.3 \mathrm{~A} \mathrm{~g} \mathrm{~g}^{-1}$ and excellent rate performance $\left(308 \mathrm{~F} \mathrm{~g}^{-1}, 87 \%\right.$ of initial value, even at $1.7 \mathrm{~A} \mathrm{~g}^{-1}$ ).

The rise in the research interest in wearable electronics has continuously stimulated the fast improvement of flexible supercapacitors with good electrochemical performance and mechanical robustness. ${ }^{118}$ Sun et al. reported the laser writing of flexible planar supercapacitors using graphene oxide (GO) and BP QD composites. The BP QDs prepared through the ultrasonic treatment of bulk BP was cooperated with GO to form uniform GO-BPQD precursor, taking advantage of the presence of abundant oxygen functional groups on the surface of the GO nanosheet, and then the obtained mixture was dropcasted on the oxygen plasma-treated polydimethylsiloxane (PDMS) substrate. After the employment of the direct laser writing technology, an apparent color change could be identified along with the reduction of GO, leaving the reduced GO-BPQD electrode, which was assembled into flexible supercapacitors by the facile coating of the $\mathrm{PVA}-\mathrm{H}_{3} \mathrm{PO}_{4}$ electrolyte (Fig. 11c). ${ }^{112}$ Notably, no obvious degradation of the electrochemical performance was observed even after blending 1000 times. Yu and his co-workers prepared a kind of BP sponge in a twoelectrode H-type electrolytic cell with a cation-exchange membrane in the middle of the bridge, using bulk BP as the cathode, Pt film as the counter electrode, and tetrabutylphosphonium bromide as the electrolyte. ${ }^{113}$ The prepared electrode was then infiltrated with a gel electrolyte of poly(vinylalcohol)/ phosphoric acid $\left(\mathrm{PVA} / \mathrm{H}_{3} \mathrm{PO}_{4}\right)$ and pressed together with the other one to assemble a symmetric all-solid-state supercapacitor (Fig. 11d), which demonstrated a high capacitance of $80 \mathrm{~F} \mathrm{~g}^{-1}$ at $10 \mathrm{mV} \mathrm{s}^{-1}$ and good reversibility (80\% retention after 15000 charging/discharging cycles). ${ }^{113}$ Zhang et al. designed an interesting self-standing polypyrrole/BP (PPy/BP) laminated film via a facile one-step electrochemical deposition strategy. Under vigorous stirring and sonication, the pyrrole monomer and BP nanosheets were mixed in the electrolyte, during which the BP sheets could be 
captured by the chains of the monomer and driven onto the ITO surface under constant potential deposition. ${ }^{114}$ Furthermore, the duration of this electrochemical deposition could be optimized to $400 \mathrm{~s}$ and the collected film was easy to be peeled off and robust enough to be folded into a paper plane, indicating promising potential for the fabrication of flexible electronic supercapacitors. Sandwich-structured supercapacitors were assembled by pressing two pieces of the PPy/BP-laminated film electrodes with the $\mathrm{H}_{3} \mathrm{PO}_{4}$-PVA gel electrolyte spread onto the surface, which exhibited an impressive capacitance of $452.8 \mathrm{~F} \mathrm{~g}^{-1}\left(7.7 \mathrm{~F} \mathrm{~cm}^{-3}\right)$, excellent cycling durability for 10000 cycles, and reliable flexibility after bending 3000 times (Fig. 11e). ${ }^{114}$ The in situ reaction between the $2 \mathrm{D}$ sheets of BP and graphene oxide was explored by Derby's group. Typically, GO dispersion made by modified Hummers' method was mixed with the BP flake suspension, and the mixture was then subjected to sonication and vacuum filtration with a PTFE filter membrane, forming the GO/BP hybrid film. The hybrid was finally exposed to high humidity to trigger the transformation into the $\mathrm{RGO} / \mathrm{H}_{x} \mathrm{PO}_{y}$ film by means of the in situ redox reaction between BP and GO. ${ }^{119}$ When the obtained $\mathrm{RGO} / \mathrm{H}_{x} \mathrm{PO}_{y}$ was assembled into the supercapacitor and tested at a scan rate of $100 \mathrm{mV} \mathrm{s}^{-1}$, it could be revealed that the CV curve of the hybrid RGO supercapacitor deteriorated significantly from the ideal, while that of the $\mathrm{RGO} / \mathrm{H}_{x} \mathrm{PO}_{y}$ supercapacitor still maintained a rectangular shape, suggesting the existence of abundant channels for ion diffusion by the presence of $\mathrm{H}_{x} \mathrm{PO}_{y}$ acids, which successfully avoided sluggish ion transport in the densely restacked RGO film (Fig. 11f). Tian and his co-workers reported that the restacking of liquid-exfoliated $\mathrm{BP}$ nanoflakes synthesized by the sonication of the BP powders in anhydrous acetone was a facile way for the assembling of flexible electrodes in all-solidstate supercapacitors with the $\mathrm{PVA} / \mathrm{H}_{3} \mathrm{PO}_{4}$ gel electrolyte sandwiched (Fig. 11g). Excellent performances of electrochemical energy storage have been achieved, including high stack capacitance up to $13.75 \mathrm{~F} \mathrm{~cm}^{-3}\left(45.8 \mathrm{~F} \mathrm{~g}^{-1}\right)$ at a scan rate of $10 \mathrm{mV} \mathrm{s}^{-1}$, as well as outstanding mechanical flexibility and cycling stability (15.5\% capacitance decay after 10000 cycles and $28.2 \%$ after 30000 cycles). ${ }^{115}$ After one year, the same group proposed a similar all-solid-state supercapacitor based on flexible BP nanoflake/carbon nanotube composite paper. Carbon nanotubes were added to act as the active material, which could enhance the conductivity of the hybrid electrode, promote ion shuttling, and avoid restacking between the BP nanoflakes. ${ }^{116}$ The CV curves measured at $0.5 \mathrm{~V} \mathrm{~s}^{-1}$ (Fig. 11h) with the fabricated supercapacitor bent at various angles $\left(0-120^{\circ}\right)$ implied the excellent flexibility and reliability of the proposed all-solid-state supercapacitor. A new single elemental hybrid composed of BP and RP (denoted as the BP/RP hybrid) was prepared by Zhong et al. via a simple sonochemical process, which was carried out using the ultrasonic cell smash, then treated in a repeated sonication bath. ${ }^{117}$ The co-existing $\mathrm{BP}$ and RP resulted in a kind of unique single elemental heterostructure with good interfacial contact, which is beneficial for electron transfer and further supercapacitor application. As exhibited in Fig. 11i, the BP/RP hybrid-based supercapacitor achieved a high specific capacitance of about $60.1 \mathrm{~F} \mathrm{~g}^{-1}$ and an impressive cycling stability with a capacity retention of $83.3 \%$ after 2000 cycles. $^{117}$ This research paves a facile way for the design of BP-based high-performance supercapacitors. Various BP-based active materials that have been applied in supercapacitors are described in Table 2.

\section{Application in the energy conversion process}

\subsection{Photocatalysis}

5.1.1 Photocatalytic hydrogen generation. Layered BP has been applied as a catalyst in the field of photocatalytic water splitting for $\mathrm{H}_{2}$ generation due to its remarkable properties, such as high hole mobility, strong optical absorption, and tunable band gap. In 2016, Lei et al. prepared a novel hybrid photocatalyst with $\mathrm{Ag}$ nanoparticles supported on BP nanosheets through chemical reduction. ${ }^{133}$ The $\mathrm{Ag}$ nanoparticles were anchored on BP via covalent bonds at the Ag/ $\mathrm{BP}$ interface and $\mathrm{Ag}-\mathrm{Ag}$ interactions, and a significant rise in the photoactivity could be identified. Besides, the decrease in the BP layer thickness from multiple layers to few layers could also promote the generation of strongly oxidative holes and further enhance the photoactivity. Visible light photocatalytic $\mathrm{H}_{2}$ evolution of the BP nanosheets was also optimized by milling bulk BP together with various additives, such as $\mathrm{LiOH}$ and NaCl. Significantly, hydroxyl-functionalized BP nanosheets were collected by LiOH modification, which demonstrated an

Table 2 Summary of the performance of BP-based supercapacitors

\begin{tabular}{|c|c|c|c|c|c|}
\hline Name & Electrolyte & Specific capacity & Current or scan rate & Cycling retention & Ref. \\
\hline BP/PANI composite & $\mathrm{H}_{2} \mathrm{SO}_{4}$ & $354 \mathrm{~F} \mathrm{~g}^{-1}$ & $0.3 \mathrm{~A} \mathrm{~g}^{-1}$ & $96 \%$ after 175 cycles & 111 \\
\hline Exfoliated BP nanoflakes & $\mathrm{PVA} / \mathrm{H}_{3} \mathrm{PO}_{4}$ & $308.7 \mathrm{~F} \mathrm{~cm}^{-3}$ & $0.01 \mathrm{~V} \mathrm{~s}^{-1}$ & $71.8 \%$ after 30000 cycles & 115 \\
\hline BP/CNT composite & $\mathrm{PVA} / \mathrm{H}_{3} \mathrm{PO}_{4}$ & $41.1 \mathrm{~F} \mathrm{~cm}^{-3}$ & $0.005 \mathrm{~V} \mathrm{~s}^{-1}$ & $91.5 \%$ after 10000 cycles & 116 \\
\hline 3D BP sponge & $\mathrm{PVA} / \mathrm{H}_{3} \mathrm{PO}_{4}$ & $80 \mathrm{~F} \mathrm{~g}^{-1}$ & $10 \mathrm{mV} \mathrm{s}^{-1}$ & $80 \%$ after 15000 cycles & 113 \\
\hline R-GO-BPQDs hybrid & $\mathrm{PVA} / \mathrm{H}_{3} \mathrm{PO}_{4}$ & $5.6 \mathrm{mF} \mathrm{cm}^{-2}$ & $5 \mathrm{mV} \mathrm{s}^{-1}$ & $98.1 \%$ after 1000 cycles & 112 \\
\hline $\mathrm{GO} / \mathrm{BP}$ hybrid & $\mathrm{H}_{x} \mathrm{PO}_{y}$ & $104.4 \mathrm{~F} \mathrm{~g}^{-1}$ & $0.25 \mathrm{~A} \mathrm{~g}^{-1}$ & ca. $80 \%$ after 5000 cycles & 119 \\
\hline $\mathrm{R}-\mathrm{BP} / \mathrm{SPC}$ & $\mathrm{H}_{2} \mathrm{SO}_{4}$ & $364.5 \mathrm{~F} \mathrm{~g}^{-1}$ & $0.5 \mathrm{~A} \mathrm{~g}^{-1}$ & $89 \%$ after 10000 cycles & 121 \\
\hline $\mathrm{SiO}_{2}-\mathrm{PANI} / \mathrm{BP}$ & $\mathrm{H}_{2} \mathrm{SO}_{4}$ & $275 \mathrm{~F} \mathrm{~g}^{-1}$ & $1.0 \mathrm{~A} \mathrm{~g}^{-1}$ & $92 \%$ after 3000 cycles & 122 \\
\hline
\end{tabular}


impressive hydrogen evolution rate of $512 \mu \mathrm{mol} \mathrm{h}^{-1} \mathrm{~g}^{-1}$, approximately 20 times higher than that of bulk BP $\left(28 \mu \mathrm{mol} \mathrm{h}{ }^{-1} \mathrm{~g}^{-1}\right)$. The enhancement was attributed to improved electron reduction ability, inhibited electron-hole recombination, and the stabilized BP structure. ${ }^{134}$ Furthermore, Liu's group introduced BPQD as a hole-migration cocatalyst of layered g- $\mathrm{C}_{3} \mathrm{~N}_{4}$ for visible-light-driven photocatalytic hydrogen generation with 7\% BP QDs coupled on the surface of g- $\mathrm{C}_{3} \mathrm{~N}_{4}$ via a high-vacuum stirring method (Fig. 12a). ${ }^{123}$ The hydrogen rates of $7 \%$ BPQD- $\mathrm{C}_{3} \mathrm{~N}_{4}$ composite were 190, 133, 90, and $10.4 \mu \mathrm{mol} \mathrm{h}{ }^{-1}$ in simulated sunlight, LED-405, LED-420, and LED-550 $\mathrm{nm}$ irradiation, respectively, which are approximately $3.5,3.6$, and 3 times higher than those of the $g-\mathrm{C}_{3} \mathrm{~N}_{4}$ sample. The result provides a novel way for the fabrication of easily oxidized BP based catalysts for hydrogen generation. As the small bandgap of bulk BP $(0.3 \mathrm{eV})$ hinders the efficient photocatalytic activity, Zou and his co-workers synthesized 2D nanojunctions based on few-layered $\mathrm{BP} / \mathrm{MoS}_{2}$. The sample was prepared via the solvothermal treatment of $\mathrm{BP}$ and $\left(\mathrm{NH}_{4}\right)_{2} \mathrm{MoS}_{4}$, which could effectively accelerate the photogenerated charge separation and result in a high activity of photocatalytic hydrogen production (Fig. 12b). ${ }^{124}$ Taking advantage of the broad absorption region and the sufficient contact interfaces, the collected $\mathrm{BP} / \mathrm{MoS}_{2}$ provided an excellent hydrogen production rate $\left(1286 \mu \mathrm{mol} \mathrm{h}{ }^{-1} \mathrm{~g}^{-1}\right)$, much higher than those of Pt-loaded BP. High photo-induced charge-carrier-separation efficiency is a key factor for the determination of the photocatalytic activity. ${ }^{135}$ Zou et al. addressed the challenge of steering the charge separation accurately by growing the $\mathrm{Co}_{2} \mathrm{P}$ co-catalyst on the edge of the BP flake to craft Co-P bonds for the enhancement of photogenerated charge-carrier transfer between the BP sheet and the $\mathrm{Co}_{2} \mathrm{P}$ cocatalyst. This led to a high photocatalytic hydrogen generation rate, 39.7 times greater than that of the bare BP flake (Fig. 12c). ${ }^{125}$ Zhang and colleagues reported that BP QDs could play the role of an active photosensitizer in a heterostructure composed of BP QDs, Au nanorods, and CdS nanowires (Fig. 12d), which provided efficient photocatalytic hydrogen generation in the solar spectrum $\left(10.1 \mathrm{mmol} \mathrm{g}^{-1} \mathrm{~h}^{-1}\right.$ under UV-vis-NIR illumination and unprecedented apparent quantum efficiency (AQE) of $2.3 \%$ at $900 \mathrm{~nm}$ irradiation), significantly better than those of CdS, BP-CdS, and Au-CdS, due to the synergistic effect of its overall photoabsorption,
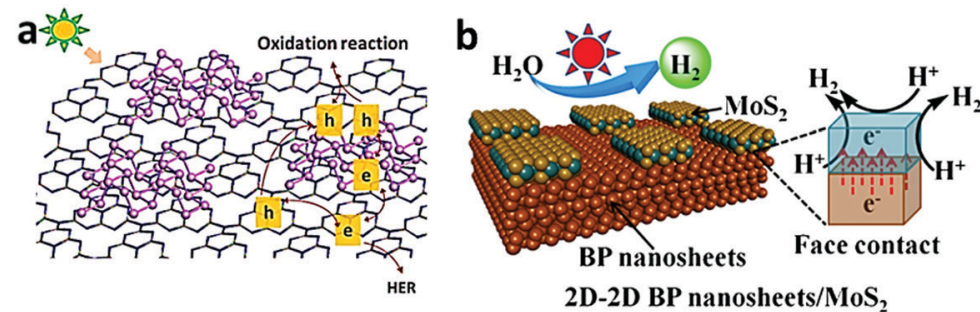

2D-2D BP nanosheets/MoS,
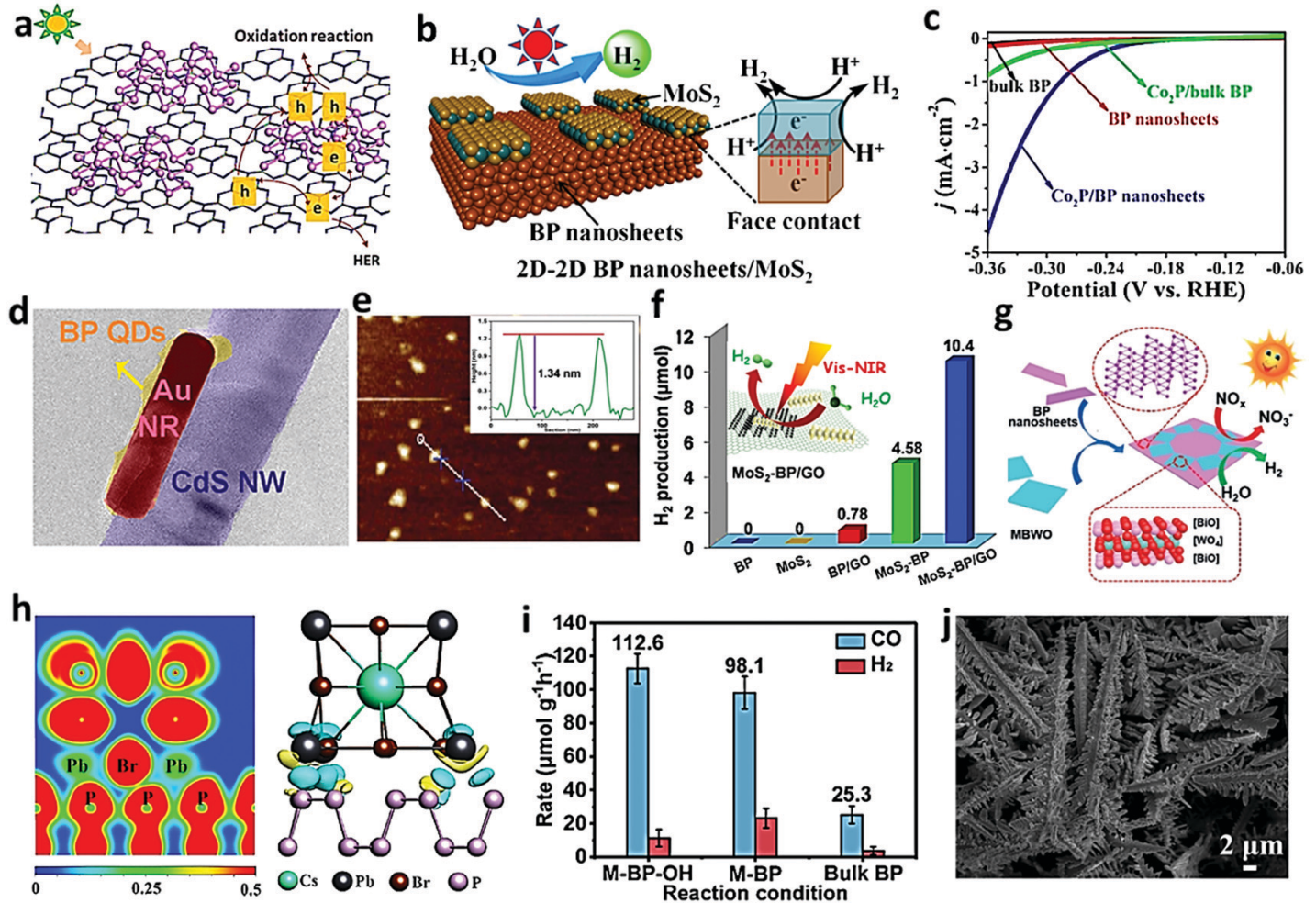

g
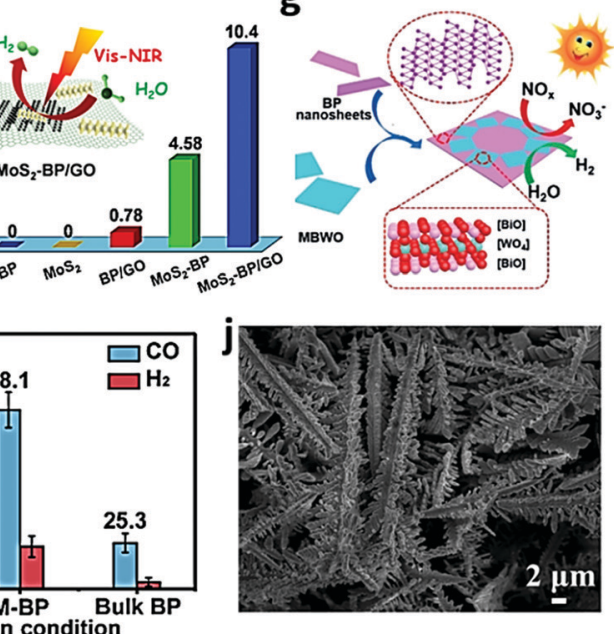

Fig. 12 The application of BP in photocatalytic hydrogen generation $(\mathrm{a}-\mathrm{g})$ and photocatalytic carbon dioxide conversion (h-j). (a) Schematic illustration of the layered $\mathrm{g}-\mathrm{C}_{3} \mathrm{~N}_{4}$ loaded with BPQD. Reproduced with permission. ${ }^{123}$ Copyright 2018, Wiley. (b) Schematic diagrams of the 2D-2D BP nanosheets/ $\mathrm{MoS}_{2}$ photocatalysts. Reproduced with permission. ${ }^{124}$ Copyright 2018, Elsevier. (c) LSV curves of different BP-based catalysts in 0.5 M sulfuric acid aqueous solution. Reproduced with permission. ${ }^{125}$ Copyright 2019, American Chemical Society. (d) TEM of the obtained BP-Au-CdS composite. Reproduced with permission. ${ }^{126}$ Copyright 2018, American Chemical Society. (e) AFM image and corresponding height profile of the obtained BP quantum dots. Reproduced with permission. ${ }^{127}$ Copyright 2019, Royal Society of Chemistry. (f) Photocatalytic $\mathrm{H}_{2}$ production of MoS 2 -BP/GO under visible-NIR light irradiation compared with other catalysts. Reproduced with permission. ${ }^{128}$ Copyright 2019, Elsevier. (g) Illustration of the fabricated BP/ MBWO heterojunction. Reproduced with permission. ${ }^{129}$ Copyright 2019, Wiley. (h) Electronic structure analysis of $\mathrm{CsPbBr}_{3} / \mathrm{BP}$. Reproduced with permission. ${ }^{130}$ Copyright 2020, Elsevier. (i) The photocatalytic activity of monolayer BP with different functional groups. Reproduced with permission. ${ }^{131}$ Copyright 2020, Elsevier. (j) SEM images of the ED-Bi dendrites/BP NSs. Reproduced with permission. ${ }^{132}$ Copyright 2020, Elsevier. 
multifunctional $\mathrm{Au}$ nanorod (electron relay and plasmonic electron donor), suitable band alignment, and stable coupling in the ternary heterostructure. ${ }^{126}$ Zeng's group successfully implanted BP QDs with a thickness of $1.34 \mathrm{~nm}$ (Fig. 12e) in the interior surface of reticulated carbon nitride materials $(\mathrm{CN}-4 \mathrm{~N})$ to form a unique structure instead of the normal exterior surface contact pattern, with the aid of the selfcapturing property of $\mathrm{CN}-4 \mathrm{~N} .{ }^{127}$ Specifically, the transparent solution of BP was first prepared by the sonication of BP powder in an ice bath and then mixed with $\mathrm{CN}-4 \mathrm{~N}$. The collected $\mathrm{CN}-4 \mathrm{~N}(\mathrm{BQ})$ was tested and achieved an impressive $\mathrm{H}_{2}$ evolution rate of $13.83 \mathrm{mmol} \mathrm{h}^{-1} \mathrm{~g}^{-1}$, which was 3.3- and 35.5-fold higher than that of $\mathrm{CN}-4 \mathrm{~N}$ and bulk $\mathrm{CN}$, respectively. The improvement was believed to result from its abundant active sites, outstanding vis-NIR absorption capability, and promoted photo-induced electron-hole separation efficiency. ${ }^{127} \mathrm{~A}$ novel and robust noble-metal-free ternary photocatalyst $\left(\mathrm{MoS}_{2}-\mathrm{BP} /\right.$ graphene oxide) was constructed by Majima et al. by mixing the $\mathrm{MoS}_{2}$ NMP dispersion with $\mathrm{BP} / \mathrm{GO}$ obtained through a solvent exfoliation method. ${ }^{128}$ After high-speed centrifugation, $\mathrm{MoS}_{2}-\mathrm{BP} / \mathrm{GO}$ was collected and further utilized in photocatalytic hydrogen production. The result showed substantial hydrogen evolution $(10.4 \mu \mathrm{mol})$ under visible-NIR light irradiation with rapid electron injection efficiency and the dual functions of graphene oxide in both synthetic and photocatalytic processes were confirmed (Fig. 12f) ${ }^{128}$ $\mathrm{Lu}$ et al. designed a Z-scheme heterojunction of the 2D/2D $\mathrm{BP} /$ monolayer $\mathrm{Bi}_{2} \mathrm{WO}_{6}(\mathrm{BP} / \mathrm{MBWO}$ ) nanosheets for photocatalysis, as assessed by hydrogen production and nitric oxide degradation at $600 \mathrm{ppb}$ under visible light illumination. The composite was fabricated by the facile sonication of the mixture of the exfoliated $\mathrm{BP}$ flake and the $\mathrm{Bi}_{2} \mathrm{WO}_{6}$ nanosheet prepared by traditional hydrothermal treatment (Fig. 12g). ${ }^{129}$ The NO removal ratio and hydrogen evolution rate of BP/MBWO were $67 \%$ and $21042 \mu \mathrm{mol} \mathrm{g}^{-1}$, approximately 2.6 and 9.15 times as high as that of pure MBWO, respectively, which could be ascribed to the interface synergy effect, fast charge separation and transfer, and good light absorbance. A kind of surface hydroxyl-modified BP was prepared by Zeng's group and used as the co-catalyst of anatase $\mathrm{TiO}_{2}$, which results in $341 \%$ photocatalytic hydrogen evolution enhancement under simulated sunlight irradiation. ${ }^{136}$ There results further indicate the potential of BP as a broadband photocatalyst.

5.1.2 Photocatalytic carbon dioxide conversion. With the increasing consumption of fossil fuel, excessive $\mathrm{CO}_{2}$ emission has brought about serious environmental issues; thus, the conversion of $\mathrm{CO}_{2}$ to useful chemical fuels is always regarded as a promising alternative strategy for the production of renewable energy. ${ }^{137,138} \mathrm{Zhu}$ et al. synthesized the heterostructure of $\mathrm{CsPbBr}_{3}$ immobilized on 2D BP $\left(\mathrm{CsPbBr}_{3} / \mathrm{BP}\right)$ by the facile self-assembly of exfoliated BP flake and colloidal $\mathrm{CsPbBr}_{3}$. With the help of direct tip sonication under bath sonication, covalent $\mathrm{Pb}-\mathrm{P}$ and $\mathrm{Br}-\mathrm{P}$ bond between $\mathrm{CsPbBr}_{3}$ and BP were clearly identified. ${ }^{130}$ In addition, charge density difference analysis was conducted to elucidate the electron transfer properties, which showed that the electrons were clearly accumulated on BP due to their transfer from $\mathrm{Pb}$ atoms in $\mathrm{CsPbBr}_{3}$. Considering the electronegativity difference between $\mathrm{Pb}$ and $\mathrm{P}$, the generated directional electron transfer channel is reasonable (Fig. 12h) ${ }^{130}$ Compared to pristine $\mathrm{CsPBBr}_{3}$, the introduction of BP significantly promoted the photocatalytic performance, exhibiting conversion rates of $\mathrm{CO}_{2}$ to $\mathrm{CO}$ $\left(44.7 \mu \mathrm{mol} \mathrm{g}^{-1} \mathrm{~h}^{-1}\right)$ and $\mathrm{CH}_{4}\left(10.7 \mu \mathrm{mol} \mathrm{g}^{-1} \mathrm{~h}^{-1}\right)$. Although BP is a promising candidate for photocatalytic carbon dioxide reduction, its limited stability remarkably impedes the application. Xu et al. successfully prepared a stable monolayer BP modified by hydroxyl (M-BP-OH) via a green exfoliation strategy assisted by liquid nitrogen. M-BP-OH was further utilized as the photocatalyst in carbon dioxide reduction, showing a photocatalytic CO production rate of $112.6 \mu \mathrm{mol} \mathrm{h}^{-1} \mathrm{~g}^{-1}$ under visible light, which is 4 times higher than that of bulk BP $\left(25.3 \mu \mathrm{mol} \mathrm{h}{ }^{-1} \mathrm{~g}^{-1}\right)$ (Fig. 12i). ${ }^{131}$ Moreover, M-BP-OH demonstrated an impressive stability even under highly humid air (90\% humidity) for $24 \mathrm{~h}$, which might be attributed to the occupation of lone pair of electrons by hydroxyl functional groups, inhibiting its reaction with oxygen. In order to explore BP nanosheets as a feasible substrate for the fabrication of metal composite catalysts for carbon dioxide reduction, bismuth was electrodeposited on the surface of the BP sheet in $0.05 \mathrm{~mol} \mathrm{dm}{ }^{-3} \mathrm{Bi}\left(\mathrm{NO}_{3}\right)_{3}$ solution under a constant voltage of $-1.0 \mathrm{~V}$, forming the $\mathrm{Bi}$ dendrites/BP composite (denoted as the ED-Bi dendrites/BP NSs) (Fig. 12j). ${ }^{132}$ The highest $\mathrm{HCOOH}$ yield rate $\left(440 \mu \mathrm{mol} \mathrm{dm}^{-3} \mathrm{~h}^{-1}\right)$ and $\mathrm{HCOOH}$ faradaic efficiency (92\%) of the ED-Bi dendrites/BP NSs were both remarkably higher than that of ED-Bi dendrites without the BP nanosheet, implying that the $\mathrm{BP}$ sheet is a desirable substrate for the deposition of metal catalyst for $\mathrm{CO}_{2}$ reduction.

5.1.3 Photocatalytic nitrogen fixation. In recent years, photocatalytic nitrogen fixation, including the absorption of solar energy and $\mathrm{N}_{2}$-to- $\mathrm{NH}_{3}$ transformation, has been regarded as a promising strategy to produce $\mathrm{NH}_{3}$ economically. ${ }^{146}$ It is of great importance to obtain low-cost and efficient metal-free cocatalysts for solar-driven photocatalytic nitrogen fixation. Zou et al. reported a desirable BP nanosheet/CdS photocatalyst (BPNS/CdS) through a two-step reaction, involving the electrochemical expansion of bulk BP and the subsequent reaction of BPNSs with $\mathrm{Cd}\left(\mathrm{CH}_{3} \mathrm{COO}\right)_{2}$ and thiourea. ${ }^{139}$ BPNS with high quality was collected by the electrochemical expansion of bulk BP in dimethyl sulfoxide (DMSO) solution containing tetra- $n$ butylammonium tetrafluoroborate (TBATFB) as the electrolyte. The gas molecules decomposed from the solvent and electrolyte and the surface hydrogenation reaction of BP could accelerate the rapid swelling and fast exfoliation. When applied for nitrogen fixation, BPNS/CdS illustrated a high $\mathrm{NH}_{3}$ generation rate of $57.64 \mu \mathrm{mol} \mathrm{L}^{-1} \mathrm{~h}^{-1}$ using the $1.5 \%$ BPNS/CdS photocatalyst, indicating the rapid photogenerated carrier separation and transfer induced by the large surface area of the BP sheets and intimate junction between BPNSs and CdS (Fig. 13a). ${ }^{139}$ Visible light-induced photocatalytic nitrogen fixation, as an environmental friendly and economical technology, requires much effort to develop efficient photocatalysts with desirable activity and stability. Jiang et al. rationally proposed a kind of metal-free BP nanosheets/graphitic carbon nitride nanosheet photocatalyst (BPCNS) by the co-sonication of the BP nanosheets suspension and the carbon nitride nanosheet (CNS) suspension. When the mass ratio of BP to CNS was 

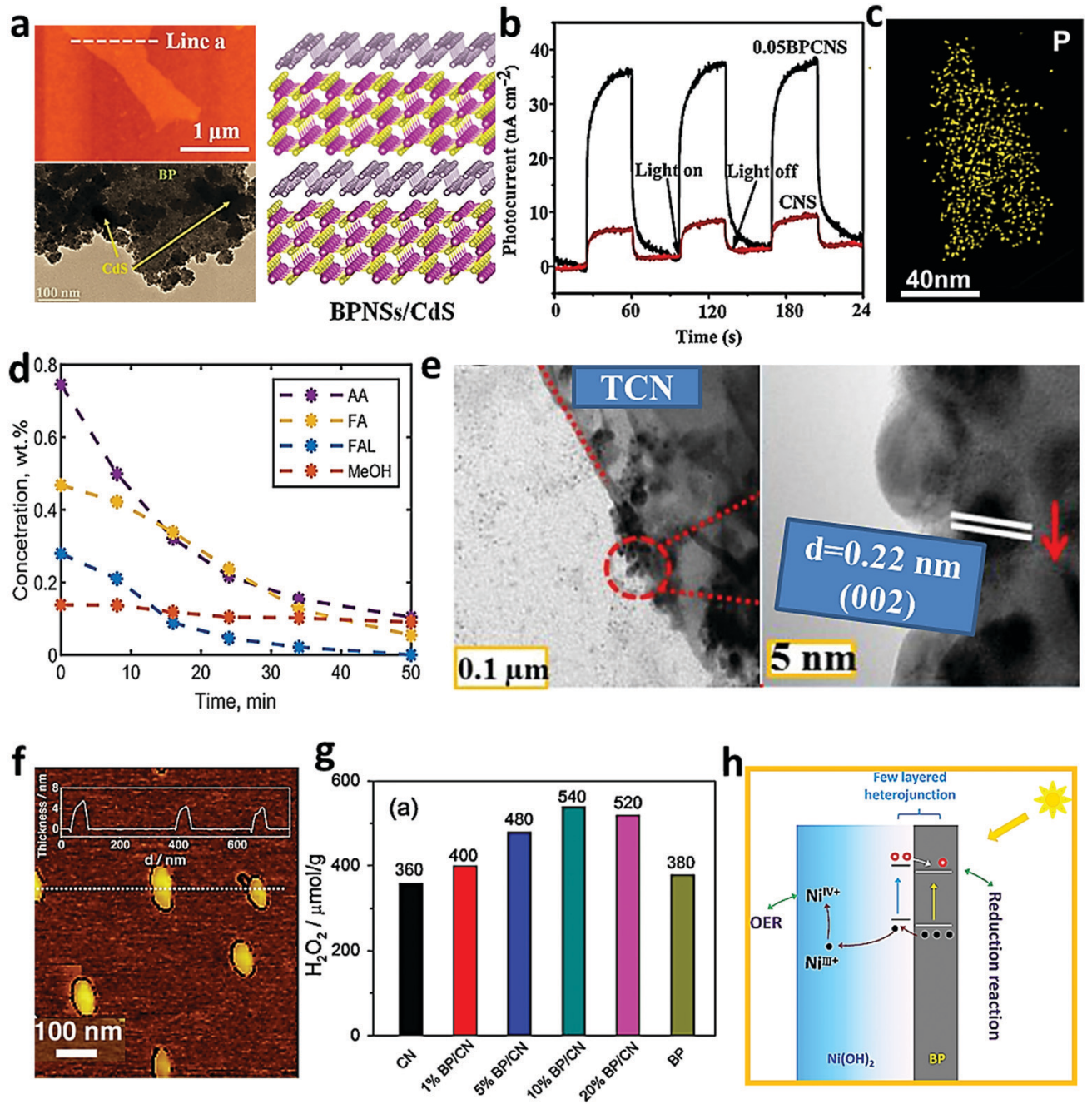

Fig. 13 The application of BP in photocatalytic nitrogen fixation (a-c), organic photodegradation (d-g) and photocatalytic water oxidation (h). (a) AFM image of BP nanosheets and TEM image of the final 1.5\% BPNS/CdS photocatalyst with schematic illustrated. Reproduced with permission. ${ }^{139}$ Copyright 2020. American Chemical Society. (b) Transient photocurrent response for CNS and 0.05BPCNS. Reproduced with permission. ${ }^{140}$ Copyright 2017 , Elsevier. (c) EDS map of an individual edge-rich BP nanoflakes. Reproduced with permission. ${ }^{141}$ Copyright 2020, American Chemical Society. (d) Wet catalytic oxidation of wastewater using robust Au/BP catalyst. Reproduced with permission ${ }^{142}$ Copyright 2020, American Chemical Society. (e) TEM images of BPTCN. Reproduced with permission. ${ }^{143}$ Copyright 2020, Elsevier. (f) AFM image and (g) photocatalytic activities of obtained BP/CN catalysts. Reproduced with permission. ${ }^{144}$ Copyright 2018, Wiley. (h) Schematic illustration and carrier transfer mechanism of the $\mathrm{P}-\mathrm{Ni}(\mathrm{OH})_{2}$ photocatalyst. Reproduced with permission. ${ }^{145}$ Copyright 2018, Wiley.

0.05, BPCNS showed a much higher activity than CNS (Fig. 13b) and a photocatalytic nitrogen fixation rate of $347.5 \mu \mathrm{mol} \mathrm{L} \mathrm{L}^{-1} \mathrm{~h}^{-1}$, which is 8.6 times as high as that of CNS $\left(40.5 \mu \mathrm{mol} \mathrm{L} \mathrm{L}^{-1} \mathrm{~h}^{-1}\right)$. The improvement was believed to result from the enhanced BPCNS excitation, inhibited recombination of the photogenerated charge carriers, and the suppressed oxidation of $\mathrm{BP}$ induced by the formation of the $\mathrm{C}-\mathrm{P}$ bond. ${ }^{140}$ To further promote the design of efficient photocatalysts for $\mathrm{NH}_{3}$ synthesis under ambient conditions, Yu and co-workers explored edge-rich BP nanoflakes (eBP NFs) for photocatalytic nitrogen fixation through facile stirring bulk BP powders, (2-bromoethyl)benzene (2-BrB), and $\mathrm{KOH}^{141}$ After chemical etching, edge-rich BP nanosheets with a flake-like shape and edge-rich rugged surface (Fig. 13c) were collected. The nitrogen fixation rate was as high as $2.37 \mathrm{mmol} \mathrm{h}^{-1} \mathrm{~g}^{-1}$ under visible-light irradiation without any cocatalysts. ${ }^{141}$ This research discloses the enhanced electron transfer from edge-rich BP nanosheets to nitrogen for hydrogenation and further reveals its great potential in "green" photocatalysis.

5.1.4 Organic photodegradation. Recently, organics have brought about severe environmental pollution and photodegradation has been regarded as an appealing way to resolve the pollution issue, taking advantage of the redox reaction of photocatalysts. BP with tunable bandgap and wide absorption wavelength range has therefore attracted great attention. Sofer's group designed a facile strategy to synthesize gold nanoparticles anchored on 2D few-layered BP by the mild mixing of tetrachloroauric acid with BP before fast centrifugation. ${ }^{142}$ The catalysis tested at $230{ }^{\circ} \mathrm{C}$ with the same amount of the proposed nanohybrid catalyst for the wet 
oxidation of acrylic acid exhibited the expected curve of decomposition, which indicated the satisfactory catalytic activity of both BP and gold nanoparticles for catalytic and environmental applications (Fig. 13d). Tang et al. introduced metal-free BP QD-loaded tubular $\mathrm{g}-\mathrm{C}_{3} \mathrm{~N}_{4}$ (denoted as BPTCN) as a promising sunlight-driven metal-free photocatalyst for oxytetracycline hydrochloride degradation and hexavalent chromium reduction in water and wastewater. In this system, commercial BP QDs were mixed with tubular $\mathrm{g}-\mathrm{C}_{3} \mathrm{~N}_{4}$ suspension, forming well-confined BPTCN heterostructure with robust BP QDs dispersed on the tubular $\mathrm{g}-\mathrm{C}_{3} \mathrm{~N}_{4}$ (Fig. 13e), providing unusual photocatalytic efficiency for the degradation of oxytetracycline hydrochloride and the reduction of hexavalent chromium. ${ }^{143}$ Wang et al. also constructed a kind of composite photocatalyst coupling carbon nitride with BP through a facile water bath sonication of carbon nitride powders and typical amounts of BP powders in $\mathrm{N}$-methyl-2-pyrrolidone (NMP) solvent. ${ }^{144}$ As the rational combination of $\mathrm{BP}$ and carbon nitride with a thickness of $4.2 \pm 1.0 \mathrm{~nm}$ (Fig. 13f) could effectively enhance the visiblelight harvesting capability, charge separation in the photocatalytic process, and photoinduced molecular oxygen activation, $98 \%$ of rhodamine B pollutant could be decomposed in $15 \mathrm{~min}$, almost four times higher than the rate with pristine carbon nitride. The amount of $\mathrm{H}_{2} \mathrm{O}_{2}$ formed on $10 \% \mathrm{BP} /$ carbon nitride was $540 \mu \mathrm{mol} \mathrm{g}^{-1}$, which was higher than that of pristine carbon nitride $\left(360 \mu \mathrm{mol} \mathrm{g}^{-1}\right)$ and BP nanosheets $\left(380 \mu \mathrm{mol} \mathrm{g}^{-1}\right)$ (Fig. 13g). ${ }^{144}$

5.1.5 Photocatalytic water oxidation. Photocatalytic water oxidation by constructing novel heterostructures with BP dots as the photocatalyst loaded on the surface of $\mathrm{Ni}(\mathrm{OH})_{2}$ was reported by Yamashita's group for the first time. ${ }^{145}$ The monolayer $\mathrm{Ni}(\mathrm{OH})_{2}$ semiconductor with reliable oxidation capability from $\mathrm{Ni}^{\mathrm{II}}$ to $\mathrm{Ni}^{\mathrm{III} / \mathrm{IV}}$ was suitable for optimal $\mathrm{O}_{2}$ evolution and the few-layered BP with a small size of ca. $10 \mathrm{~nm}$ could be utilized to ensure a suitable valence band position for water oxidation in argon-saturated $0.1 \mathrm{M} \mathrm{Na}_{2} \mathrm{~S}_{2} \mathrm{O}_{4}$ solution (Fig. 13h). It revealed a satisfactory $\mathrm{O}_{2}$ evolution rate $\left(15.7 \mathrm{mmol} \mathrm{g} \mathrm{g}^{-1} \mathrm{~h}^{-1}\right)$ under simulated sunlight irradiation.

\subsection{Electrocatalysis}

5.2.1 Electrocatalytic hydrogen evolution. In recent years, much effort has been made to explore high performance electrocatalyst for hydrogen evolution reaction (HER) as there is a growing demand for hydrogen energy. ${ }^{156}$ Since Pt-based noble metal materials with good catalytic performance for HER are limited by the high price and rare reserves, developing highefficiency and cost-effective alternative catalysts has become a requirement. Pumera et al. reported a single-step solutionbased electrochemical exfoliation for the preparation of downsized layered BP with the distance between the two-platinum electrodes and applied direct current set to $2 \mathrm{~cm}$ and $10 \mathrm{~V}$ for 30 min, respectively. ${ }^{147}$ After standing for one week, the transparent solution-containing BP nanoparticles (around $70 \mathrm{~nm}$ ) (Fig. 14a) was desalinated using the dialysis membrane and utilized for electrocatalysis. The downsized BP nanoparticles demonstrated significantly optimized electrocatalytic activity, with an overpotential of $-0.81 \mathrm{~V}$, which was apparently enhanced compared with those of BP macroparticles and glassy carbon $\left(-1.24\right.$ and $-0.97 \mathrm{~V}$, respectively). ${ }^{147}$ Zhang's group reported a rationally proposed $2 \mathrm{D}$ BP-supported $\mathrm{Ni}_{2} \mathrm{P}$-based hybrid catalyst, which was synthesized through the one-pot thermal decomposition of nickel acetylacetonate, BP, and trioctylphosphine. After washing and vacuum drying, the collected $\mathrm{Ni}_{2} \mathrm{P} / \mathrm{BP}$ was systematically characterized, indicating a uniform small size supported on the surface of the layered BP and exhibiting satisfactory HER electrocatalytic performance with low onset overpotential (70 mV), small Tafel slope ( $\left.81 \mathrm{mV} \mathrm{dec}^{-1}\right)$, and large double-layer capacitance $\left(1.24 \mathrm{mF} \mathrm{cm}^{-2}\right.$ ) (Fig. 14b) ${ }^{148}$ Zeng and co-workers deposited catalytically-active $\mathrm{MoS}_{2}$ flakes on the surface of liquid-exfoliated BP sheet to fabricate the $\mathrm{MoS}_{2}-\mathrm{BP}$ interface and modify the electronic properties, in which the electrons could transfer from the BP to $\mathrm{MoS}_{2}$ due to the higher Fermi level of BP. ${ }^{149}$ Owing to the electron donation of $\mathrm{BP}$, the accumulated electrons on $\mathrm{MoS}_{2}$ could effectively promote the coupling of electrons with protons at the catalyst-electrolyte interface, leading to satisfactory HER behavior with an overpotential of $85 \mathrm{mV}$ at $10 \mathrm{~mA} \mathrm{~cm}{ }^{-2}$ and an exchange current density of $0.66 \mathrm{~mA} \mathrm{~cm}{ }^{-2}, 22$ times higher than that of bare $\mathrm{MoS}_{2}$ (Fig. 14c). ${ }^{149}$ Although 2D BP has attracted much attention for electrocatalysis due to its semi-conductive property, high carrier mobility, and tunable bandgap, the activity of bare BP is still lower than that of other non-precious electrocatalysts. Chen's group reported a facile and green solid exfoliation approach, utilizing urea-assisted mechanical milling to synthesize $\mathrm{NH}_{2}$-modified $\mathrm{BP}$ sheets ${ }^{150}$ Typically, BP powder and $\mathrm{CO}\left(\mathrm{NH}_{2}\right)_{2}$ were milled in argon protection and the adsorption of urea on BP could weaken the layer interaction, thus promoting the exfoliation (Fig. 14d). After urea removal, the obtained $\mathrm{NH}_{2}$ - $\mathrm{BP}$ nanosheet showed an enhanced electrocatalytic HER performance with an overpotential of $290 \mathrm{mV}$ at $-10 \mathrm{~mA} \mathrm{~cm} \mathrm{~cm}^{-2}$, a Tafel slope of $63 \mathrm{mV} \mathrm{dec}^{-1}$, and a turnover frequency of $3.21 \mathrm{~s}^{-1}$ at an overpotential of $290 \mathrm{mV}$, which is much better than those of bulk BP. ${ }^{150}$

5.2.2 Electrocatalytic oxygen evolution. Oxygen evolution reaction (OER) is a crucial part of various electrochemical applications but the development of water oxidation catalysts with high activity and desirable stability is still challenging. ${ }^{157}$ Wang et al. synthesized orthorhombic BP on a Ti foil and CNT matrix by a novel thermal-vaporization transformation approach, which is a useful strategy for the synthesis of BP with different forms (e.g., BP thin film and BP particles on the substrate). ${ }^{151}$ The onset potential for OER on BP-CNT with uniform dispersion of the BP within the CNT network (Fig. 14e) was $1.49 \mathrm{~V}$, similar to that of $\mathrm{BP}-\mathrm{Ti}$, which indicated that $\mathrm{BP}$ had a major contribution in OER. BP-CNT only required $1.6 \mathrm{~V}$ to deliver a $10.0 \mathrm{~mA} \mathrm{~cm}{ }^{-2}$ current density close to that of commercial $\mathrm{IrO}_{2}(1.57 \mathrm{~V})$ and commercial $\mathrm{RuO}_{2}(1.59 \mathrm{~V})$, verifying the effective electrocatalytic capability. ${ }^{151} \mathrm{Yu}$ et al. designed a facile solvothermal strategy to prepare in-plane $\mathrm{BP} /$ dicobalt phosphide (denoted as $\mathrm{BP} / \mathrm{Co}_{2} \mathrm{P}$ ) composite through the defect/edge-selective growth of $\mathrm{Co}_{2} \mathrm{P}$ on the $\mathrm{BP}$ nanosheets, during which the unsaturated $\mathrm{P}$ in the defects was inevitably produced on the BP nanosheets and cobalt with a 
a

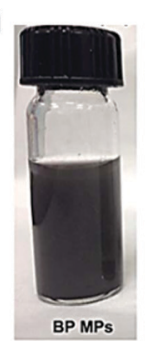

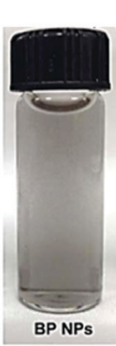
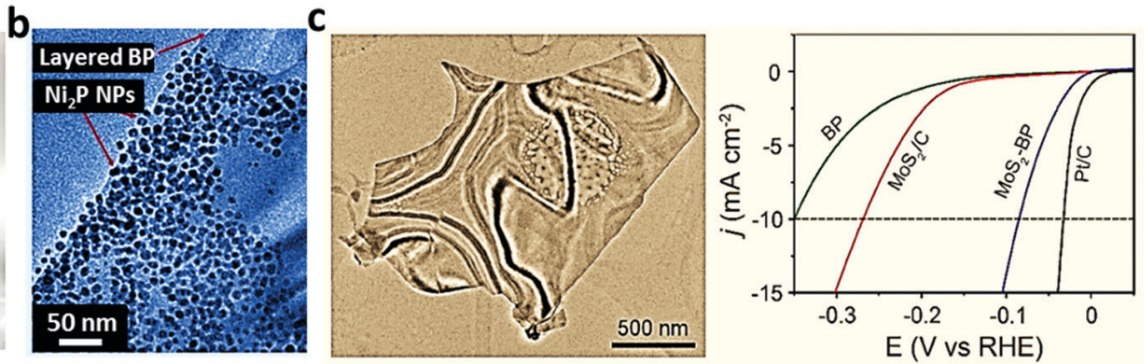

d

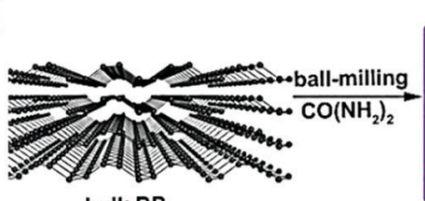

bulk BP

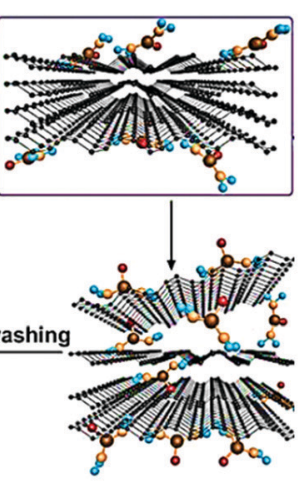

e
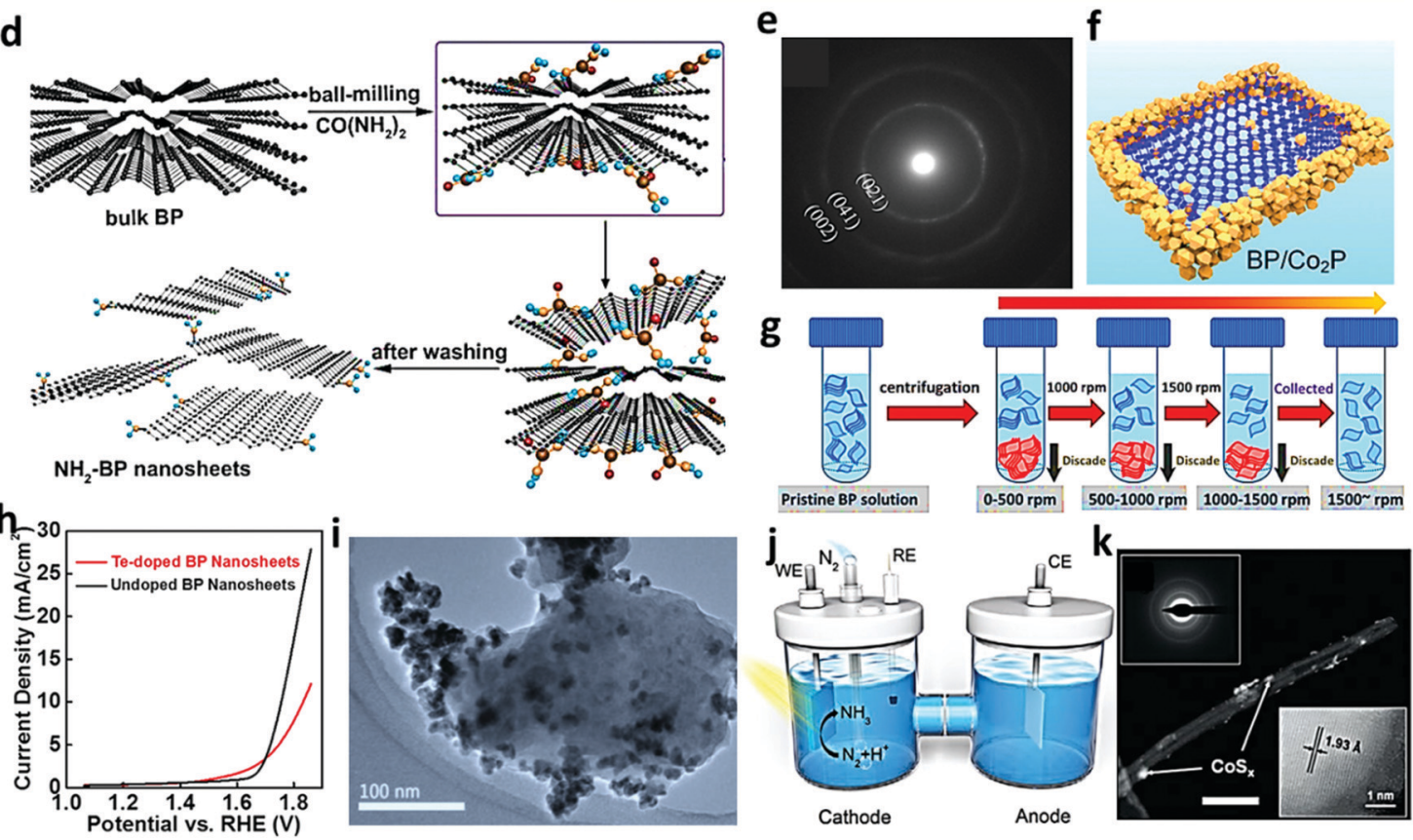

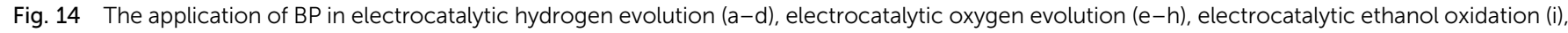

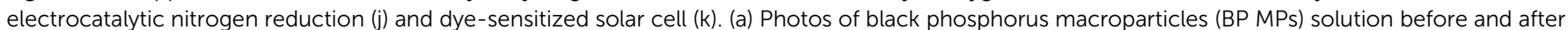

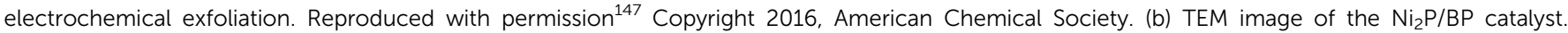

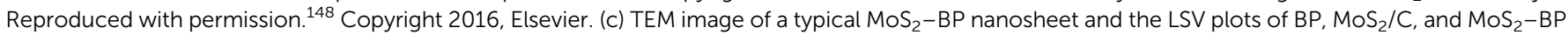

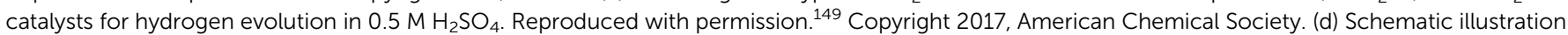

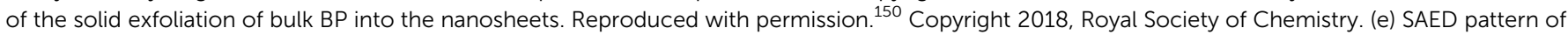

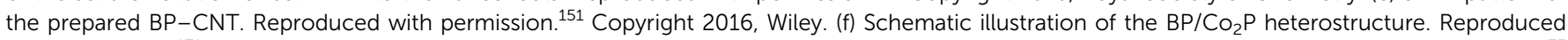

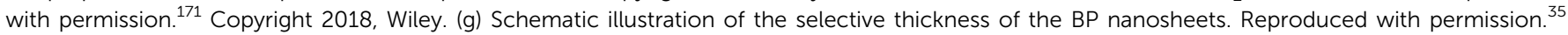

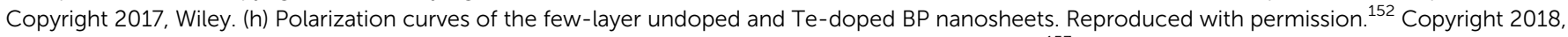

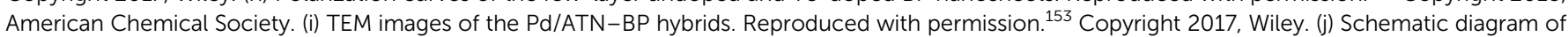

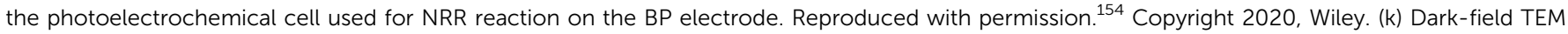

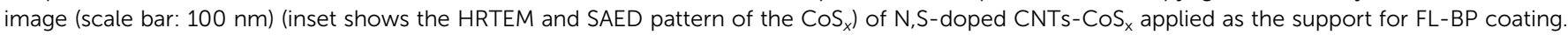
Reproduced with permission. ${ }^{155}$ Copyright 2018, Wiley.

defect occupation energy of Co $(-7.65 \mathrm{eV})$ was spontaneously generated and bonded with phosphorus (Fig. 14f). ${ }^{158}$ When OER was tested in $1.0 \mathrm{M} \mathrm{KOH}$ at $5 \mathrm{mV} \mathrm{s}{ }^{-1}$, the $\mathrm{BP} / \mathrm{Co}_{2} \mathrm{P}$ composite with an overpotential of $300 \mathrm{mV}$ and Tafel slope of $78 \mathrm{mV} \mathrm{dec}^{-1}$ needed a small voltage (517 mV) to drive $100 \mathrm{~mA} \mathrm{~cm}^{-2}$, implying the superior OER activity of the designed $\mathrm{BP} / \mathrm{Co}_{2} \mathrm{P}$. Zhang et al. sealed and ultrasonicated $40 \mathrm{mg}$ bulk BP in a conical tube. After centrifugation, BP nanosheets were collected and subjected to further characterization, demonstrating preferable OER activity (onset-potential and Tafel slope of the BP nanosheets are $1.45 \mathrm{~V}$ and $88 \mathrm{mV} \mathrm{dec}{ }^{-1}$, respectively) (Fig. 14g). ${ }^{35}$ In addition, it was also concluded that the reduction in the thickness of $\mathrm{BP}$ would result in extra active sites, which is beneficial for the enhancement of electrocatalytic activities. This work might provide further implementation of the OER performance of the BP nanosheets. Yan's group prepared the Te-doped BP nanosheets by the co-calcination of $\mathrm{RP}$, tin, and tellurium iodide $\left(\mathrm{TeI}_{4}\right)$, followed by the ultrasonication of the bulk BP. ${ }^{152}$ Owing to the change in the charge distribution and electronic properties induced by the introduction of Te atoms with different radius and electronegativity, the electrocatalytic activity of the Te-doped BP sheet was significantly optimized, showing a lower onset potential (about 1.49 V) (Fig. 14h).

5.2.3 Electrocatalytic ethanol oxidation. Fuel cells converting chemical energy to electrical energy have been constantly explored for green power generation, among which direct ethanol fuel cells 
(DEFCs) with high current density are extremely prominent. Therefore, the design and preparation of excellent DEFC-based catalysts have attracted increasing attention. ${ }^{159}$ Min et al. proposed a novel anatase $\mathrm{TiO}_{2}$ nanosheets-BP (ATN-BP) hybrid as a support for Pd nanoparticles used in the ethanol oxidation reaction (EOR) (Fig. 14i). ${ }^{153}$ The ATN-BP composite was synthesized by the ball-milling of BP and ATN, which was then slowly combined with $\mathrm{Na}_{2} \mathrm{PdCl}_{4}$ with the assistance of $\mathrm{KBH}_{4}$. After suction filtration and thorough washing, Pd/ATN-BP was collected and provided a high EOR activity with a mass peak current density of $5023.8 \mathrm{~mA} \mathrm{mg}^{-1}$, which was ascribed to the formation of $\mathrm{P}-\mathrm{O}-\mathrm{Ti}$ bonds and further electron accumulation in the anchored Pd nanoparticles. ${ }^{153}$

5.2.4 Electrocatalytic nitrogen reduction. Ammonia is an important chemical in industries and agriculture, and several electrocatalytic methods have been developed to produce ammonia from nitrogen under ambient conditions as an alternative to the traditional Haber-Bosch ammonia synthesis. ${ }^{160,161}$ Owing to the abundant active sites and weak hydrogen absorption, BP maintains promising potential in nitrogen reduction reaction (NRR), the core process in ammonia production. Yu et al. fabricated an electrode through the layer-by-layer assembly of BP nanosheets on an indium tin oxide (ITO) substrate, during which ethanol dispersion of the BP sheets was spun and then heated on a hot plate. BP electrodes (120 nm, $0.1 \mathrm{mg} \mathrm{cm}^{-2}$ ) were finally obtained, demonstrating an ammonia yield rate of $102.4 \mu \mathrm{g} \mathrm{h}^{-1} \mathrm{mg}_{\text {cat }}{ }^{-1}$ in the photoelectrochemical (PEC) cell, faradaic efficiency of $23.3 \%$ at $-0.4 \mathrm{~V}$, and excellent stability after 6 consecutive cycles (Fig. 14j). ${ }^{154} \mathrm{Zhu}$ et al. reported a facile strategy for the self-assembly of BP QDs on the $\mathrm{MnO}_{2}$ flake via van der Waals interactions (BP QDs/ $\mathrm{MnO}_{2}$ ). The ultrafine size of the BP QDs was beneficial for the utilization of the active sites, while the cooperation of the graphene-like $\mathrm{MnO}_{2}$ sheet as a high-efficiency substrate could inhibit the BP QDs from aggregating and fulfill the robust combination. The obtained double active composite maintained appealing synergy effect toward nitrogen fixation and reached a $\mathrm{NH}_{3}$ yield rate of $25.3 \mu \mathrm{g} \mathrm{h}^{-1} \mathrm{mg}^{-1}$ and a faradaic efficiency of $6.7 \%$ with $0.1 \mathrm{M} \mathrm{Na}_{2} \mathrm{SO}_{4}$ electrolyte under ambient conditions, further enlightening the effective constructing of the BP-based material for nitrogen fixation. ${ }^{162}$ QDs with large surface area and abundant active sites are prone to agglomeration, resulting in the loss of active sites. To address this issue, $\mathrm{SnO}_{2-x}$ nanotubes were proposed by Ding et al. through controlled etching and heat treatment of the $\mathrm{SnO}_{2} @ \mathrm{SiO}_{2}$ nanorods with $\mathrm{NaOH}$ and $\mathrm{NaBH}_{4}$, respectively. BP QDs collected through facile sonication were assembled onto the $\mathrm{SnO}_{2-x}$ nanotubes with the help of spontaneous $\mathrm{Sn}-\mathrm{P}$ coordination, leading to a stable, double-active electrocatalyst. ${ }^{163}$ When utilized for the nitrogen fixation test, the proposed $\mathrm{BP} @ \mathrm{SnO}_{2-x}$ provided a high ammonia yield (48.87 $\mu \mathrm{g} \mathrm{h}^{-1} \mathrm{mg}^{-1}$ ) and satisfactory faradaic efficiency (14.6\%) at $-0.4 \mathrm{~V}$, representing a valuable attempt toward low-cost and efficient BP based catalysts for ambient $\mathrm{N}_{2}$ fixation.

5.2.5 Dye-sensitized solar cell. Dye-sensitized solar cell (DSSC) is another important application of BP-based electrocatalysts. Shapter et al. demonstrated the effective production of BP nanosheets through microwave-assisted liquid-phase exfoliation. The BP nanosheets were applied in photoelectrochemical cells, which were found to act as catalytically active sites and to illustrate the desirable electrocatalytic activity for triiodide reduction in DSSCs. Notably, the device assembled using the delicately designed cobalt sulfide $\left(\mathrm{CoS}_{x}\right)$ decorated nitrogen and sulfur co-modified carbon nanotube heteroelectrocatalyst coated with FL-BP (FL-BP@N,S-doped CNTs-CoS $_{x}$ ) (Fig. 14k) exhibited an excellent photovoltaic efficiency of $8.31 \%$, paving a new way for the application of BP sheet-based electrocatalysts. ${ }^{155}$

\section{Conclusion and perspective}

In this review, the recent progress in the synthesis of black phosphorus-based active materials and their utilization in energy storage (Li-ion, Na-ion, K-ion, $\mathrm{Li}-\mathrm{S}, \mathrm{Li}-\mathrm{O}_{2}$, and $\mathrm{Zn}-\mathrm{Ni}$ batteries as well as supercapacitors) and conversion (solar cells, hydrogen evolution, carbon dioxide reduction, oxygen evolution, and nitrogen fixation) have been discussed. The progress shows a fast-growing knowledge of black phosphorus-based energy applications, together with breakthroughs in facile synthesis and other bottlenecks, such as sample quality, production scalability, and reserve stability.

Although impressive enhancements have been made in the past few years, several bottlenecks remain. The transformation from RP, the most popular phosphorus precursor for BP preparation, generally cannot be precisely controlled, let alone the surface cleanliness, which significantly influences the quality level of the final product. Since the ambient atmosphere can irreversibly convert $\mathrm{BP}$ into $\mathrm{PO}_{x}$ compounds, resulting in the degradation of the fundamental properties, the protection of few-layered BP will remarkably influence its performance in energy applications. Recently, various strategies have been adopted for surface passivation, such as $\mathrm{AlO}_{x}$ film encapsulation induced by atomic layer deposition, ${ }^{164}$ titanium sulfonate ligand coordination, ${ }^{165}$ self-passivation by the formation of single layer phosphorus oxide at the surface, ${ }^{166}$ encapsulation by selfassembly of the perylene-3,4,9,10-tetracarboxylic dianhydride monolayers, ${ }^{167}$ and formation of covalent phosphorus-carbon bonds by aryl diazonium modification. ${ }^{168}$ Further effective stability protection technologies are still needed for practical commercialization as the fabrication of air-stable 2D BP or its derivatives will continue to be a major shortcoming in the near future.

In the battery system, although the BP-based anode has high theoretical capacities (e.g., $2596 \mathrm{~mA} \mathrm{~h} \mathrm{~g}^{-1}$ in LIB with the $\mathrm{Li}_{3} \mathrm{P}$ alloy formed), the initial Coulombic efficiency, packing density, discharge-charge potential, and hysteresis of the BP-based electrode still significantly influence the energy storage performance. Initial Coulombic efficiency is a key parameter to reflect the amount of the irreversibly consumed ion, which could remarkably determine the full cell capacity. Based on the research of Chen and et al., the decreased surface area and the modified surface properties could effectively optimize the Coulombic efficiency and a high initial Coulombic efficiency of 
$84 \%$ could be achieved when the BP content in BP/active carbon was $45 \mathrm{wt} \%{ }^{169}$ Besides, electrolyte engineering is also a desirable way to increase the initial Coulombic efficiency. During the battery operation process, suitably engineered electrolyte could provide suppressed decomposition, leading to a homogeneous, thin, and stable solid electrolyte interphase layer. Moreover, the packing density of the BP-based electrodes is usually at a low level because most of the BP-active materials in the battery field have a 2D structure and a large surface area. Although it is feasible to increase the pack density by combining BP with various highdensity counterparts, the overall effect is still limited and more attempts should be made. Owing to the sluggish kinetics, voltage hysteresis usually occurs within the BP-based electrodes during the charge and discharge process, and continuously leads to irreversible capacities loss. According to Han's report, cooperating BP with highly conductive carbon nanotubes was beneficial for the alleviation of hysteresis from 0.81 to $0.72 \mathrm{~V}$; it could be attributed to the better connection between the particles in the process of large volume change. ${ }^{56}$

In terms of the energy conversion performance, three key parameters are of vital importance for the utilization of BP-based catalysts. The first one is chemical stability. The phosphorus atoms at the edge position have an unstable pair of lone electrons, which make them much vulnerable to degradation under ambient conditions. $^{172,173}$ The hybridization strategy has been used to produce heterostructured composites and tune the interfacial condition of BP, which improves its chemical stability and photocatalytic activity. However, hybridization is generally non-selective and provides insufficient protection to the $\mathrm{P}$ atoms at the edge sites. At present, suitable functional molecules, which can selectively react with the edge sites of $\mathrm{BP}$, are needed to establish the practical application of BP-based materials. The second one is the charge separation efficiency. Although coupling with a traditional noble-metal is an effective way to achieve high charge separation efficiency, exploiting a noble-metal-free catalyst, such as CoP and $\mathrm{MoS}_{2}$, has emerged as a research topic of great concern since the traditional noble metals generally have high cost. The third one is bandgap engineering. Owing to the quantum confinement of the nanosized $\mathrm{BP}$, the conduction band and valence band could gradually vary to the more negative and positive levels with the decreasing layer number, which endows $\mathrm{BP}$ with a tunable bandgap ranging from $0.3 \mathrm{eV}$ (bulk value) to $2.0 \mathrm{eV}$ (monolayer value). To achieve an appropriate catalytic capability, the thickness of BP should be precisely controlled and the cooperation with other components should be rationally designed, which poses a challenge in the synthetic procedure and technique.

All in all, with persistent attempts by researchers around the world, it is out of question that black phosphorus would not only open a new chapter for a new generation of energy materials but also provide a remarkable market potential in the foreseeable future.

\section{Conflicts of interest}

There are no conflicts to declare.

\section{Acknowledgements}

This work was supported by the National Natural Science Foundation of China under grant no. 51925804, and the Shenzhen Science and Technology Project under grant no. JCYJ20180507182246321.

\section{Notes and references}

1 M. Baba, F. Izumida, Y. Takeda and A. Morita, Jpn. J. Appl. Phys., 1989, 28, 1019.

2 C. M. Park and H. J. Sohn, Adv. Mater., 2007, 19, 2465.

3 S. Lange, P. Schmidt and T. Nilges, Inorg. Chem., 2007, 46, 4028.

4 M. Zhao, H. L. Qian, X. Y. Niu, W. Wang, L. Guan, J. Sha and Y. W. Wang, Cryst. Growth Des., 2016, 16, 1096.

5 R. Vargas-Bernal, Sensors, 2019, 19, 1295.

6 Z. Shao, C. Wang, K. Wu, H. Zhang and J. Chen, Nanoscale Adv., 2019, 1, 4190.

7 D. Tyagi, H. Wang, W. Huang, L. Hu, Y. Tang, Z. Guo, Z. Ouyang and H. Zhang, Nanoscale, 2020, 12, 3535.

8 Z.-L. Xu, S. Lin, N. Onofrio, L. Zhou, F. Shi, W. Lu, K. Kang, Q. Zhang and S. P. Lau, Nat. Commun., 2018, 9, 1.

9 Y. Jing, Z. Zhou, C. R. Cabrera and Z. Chen, J. Mater. Chem. A, 2014, 2, 12104.

10 Y. Shi, B. Li, Q. Zhu, K. Shen, W. Tang, Q. Xiang, W. Chen, C. Liu, J. Luo and S. Yang, Adv. Energy Mater., 2020, 10, 1903534.

11 R. Zhang, J. Liu, Y. Gao, M. Hua, B. Xia, P. Knecht, A. C. Papageorgiou, J. Reichert, J. V. Barth and H. Xu, Angew. Chem., Int. Ed., 2020, 132, 2691.

12 K. S. Novoselov, A. K. Geim, S. V. Morozov, D. Jiang, M. I. Katsnelson, I. V. Grigorieva, S. V. Dubonos and A. A. Firsov, Nature, 2005, 438, 197.

13 A. K. Geim and K. S. Novoselov, Nat. Mater., 2007, 6, 183. 14 F. Schwierz, Nat. Nanotechnol., 2010, 5, 487.

15 H. Li, Y. J. Zeng, X. J. Hu, H. H. Zhang, S. C. Ruan, M. J. Van Bael and C. Van Haesendonck, Carbon, 2017, 124, 193.

16 X. Y. Chia, A. Y. S. Eng, A. Ambrosi, S. M. Tan and M. Pumera, Chem. Rev., 2015, 115, 11941.

17 Q. Han, B. Wang, J. Gao and L. T. Qu, Angew. Chem., Int. Ed., 2016, 55, 10849.

18 S. L. Huang, H. Yi, L. H. Zhang, Z. Y. Jin, Y. J. Long, Y. Y. Zhang, Q. F. Liao, J. Na, H. Z. Cui, S. C. Ruan, Y. Yamauchi, T. Wakihara, Y. V. Kaneti and Y. J. Zeng, J. Hazard. Mater., 2020, 393, 122324.

19 S. T. Han, L. Hu, X. D. Wang, Y. Zhou, Y. J. Zeng, S. C. Ruan, C. F. Pan and Z. C. Peng, Adv. Sci., 2017, 4, 1600435.

20 A. L. Zhao, H. Li, X. J. Hu, C. Wang, H. Zhang, J. G. Lu, S. C. Ruan and Y. J. Zeng, J. Phys. D, 2020, 53, 293002.

21 J. Sun, G. Y. Zheng, H. W. Lee, N. Liu, H. T. Wang, H. B. Yao, W. S. Yang and Y. Cui, Nano Lett., 2014, 14, 4573.

22 L. K. Li, Y. J. Yu, G. J. Ye, Q. Q. Ge, X. D. Ou, H. Wu, D. L. Feng, X. H. Chen and Y. B. Zhang, Nat. Nanotechnol., 2014, 9, 372 . 
23 T. Nilges, M. Kersting and T. Pfeifer, J. Solid State Chem., 2008, 181, 1707.

24 M. Kopf, N. Eckstein, D. Pfister, C. Grotz, I. Kruger, M. Greiwe, T. Hansen, H. Kohlmann and T. Nilges, J. Cryst. Growth, 2014, 405, 6.

25 P. W. Bridgman, J. Am. Chem. Soc., 1914, 36, 1344.

26 R. W. Keyes, Phys. Rev., 1953, 92, 580.

27 L. Q. Sun, M. J. Li, K. Sun, S. H. Yu, R. S. Wang and H. M. Xie, J. Phys. Chem. C, 2012, 116, 14772.

28 M. Dahbi, N. Yabuuchi, M. Fukunishi, K. Kubota, K. Chihara, K. Tokiwa, X. F. Yu, H. Ushiyama, K. Yamashita, J. Y. Son, Y. T. Cui, H. Oji and S. Komaba, Chem. Mater., 2016, 28, 1625.

29 Y. Maruyama, S. Suzuki, K. Kobayashi and S. Tanuma, Physica B \& $C$, 1981, 105, 99.

30 M. Nagao, A. Hayashi and M. Tatsumisago, J. Power Sources, 2011, 196, 6902.

31 C. Suryanarayana, Prog. Mater. Sci., 2001, 46, 1.

32 W. L. Lu, H. Y. Nan, J. H. Hong, Y. M. Chen, C. Zhu, Z. Liang, X. Y. Ma, Z. H. Ni, C. H. Jin and Z. Zhang, Nano Res., 2014, 7, 853.

33 J. R. Brent, N. Savjani, E. A. Lewis, S. J. Haigh, D. J. Lewis and P. O'Brien, Chem. Commun., 2014, 50, 13338.

34 Z. B. Sun, H. H. Xie, S. Y. Tang, X. F. Yu, Z. N. Guo, J. D. Shao, H. Zhang, H. Huang, H. Y. Wang and P. K. Chu, Angew. Chem., Int. Ed., 2015, 54, 11526.

35 X. H. Ren, J. Zhou, X. Qi, Y. D. Liu, Z. Y. Huang, Z. J. Li, Y. Q. Ge, S. C. Dhanabalan, J. S. Ponraj, S. Y. Wang, J. X. Zhong and H. Zhang, Adv. Energy Mater., 2017, 7, 1700396.

36 J. Kang, J. D. Wood, S. A. Wells, J. H. Lee, X. L. Liu, K. S. Chen and M. C. Hersam, ACS Nano, 2015, 9, 3596.

37 P. Yasaei, B. Kumar, T. Foroozan, C. H. Wang, M. Asadi, D. Tuschel, J. E. Indacochea, R. F. Klie and A. SalehiKhojin, Adv. Mater., 2015, 27, 1887.

38 D. Hanlon, C. Backes, E. Doherty, C. S. Cucinotta, N. C. Berner, C. Boland, K. Lee, A. Harvey, P. Lynch, Z. Gholamvand, S. F. Zhang, K. P. Wang, G. Moynihan, A. Pokle, Q. M. Ramasse, N. McEvoy, W. J. Blau, J. Wang, G. Abellan, F. Hauke, A. Hirsch, S. Sanvito, D. D. O'Regan, G. S. Duesberg, V. Nicolosi and J. N. Coleman, Nat. Commun., 2015, 6, 8563.

39 W. C. Zhao, Z. M. Xue, J. F. Wang, J. Y. Jiang, X. H. Zhao and T. C. Mu, ACS Appl. Mater. Interfaces, 2015, 7, 27608.

40 K. S. Novoselov, A. K. Geim, S. V. Morozov, D. Jiang, Y. Zhang, S. V. Dubonos, I. V. Grigorieva and A. A. Firsov, Science, 2004, 306, 666.

41 H. Liu, A. T. Neal, Z. Zhu, Z. Luo, X. F. Xu, D. Tomanek and P. D. Ye, ACS Nano, 2014, 8, 4033.

42 P. May, U. Khan, A. O'Neill and J. N. Coleman, J. Mater. Chem., 2012, 22, 1278.

43 T. F. Jaramillo, K. P. Jorgensen, J. Bonde, J. H. Nielsen, S. Horch and I. Chorkendorff, Science, 2007, 317, 100.

44 P. Hapiot and C. Lagrost, Chem. Rev., 2008, 108, 2238-2264. 45 X. Q. Wang, P. F. Fulvio, G. A. Baker, G. M. Veith, R. R. Unocic, S. M. Mahurin, M. F. Chi and S. Dai, Chem. Commun., 2010, 46, 4487.
46 W. T. Zhang, Y. R. Wang, D. H. Zhang, S. X. Yu, W. X. Zhu, J. Wang, F. Q. Zheng, S. X. Wang and J. L. Wang, Nanoscale, 2015, 7, 10210.

47 L. Hu, J. Yuan, Y. Ren, Y. Wang, J. Q. Yang, Y. Zhou, Y. J. Zeng, S. T. Han and S. C. Ruan, Adv. Mater., 2018, 30, 1801232.

48 Z. Y. Xu, L. Hu, J. Yuan, Y. Y. Zhang, Y. J. Guo, Z. Y. Jin, F. C. Long, Y. J. Long, H. W. Liang, S. C. Ruan and Y. J. Zeng, Adv. Mater. Interfaces, 2020, 7, 1902075.

49 J. B. Smith, D. Hagaman and H. F. Ji, Nanotechnology, 2016, 27, 215602.

50 Z. B. Yang, J. H. Hao, S. G. Yuan, S. H. Lin, H. M. Yau, J. Y. Dai and S. P. Lau, Adv. Mater., 2015, 27, 3748.

51 C. Li, Y. Wu, B. C. Deng, Y. J. Xie, Q. S. Guo, S. F. Yuan, X. L. Chen, M. Bhuiyan, Z. S. Wu, K. Watanabe, T. Taniguchi, H. L. Wang, J. J. Cha, M. Snure, Y. W. Fei and F. N. Xia, Adv. Mater., 2018, 30, 1703748.

52 J. J. Pei, X. Gai, J. Yang, X. B. Wang, Z. F. Yu, D. Y. Choi, B. Luther-Davies and Y. R. Lu, Nat. Commun., 2016, 7, 10450.

53 Y. H. Xu, Z. T. Wang, Z. N. Guo, H. Huang, Q. L. Xiao, H. Zhang and X. F. Yu, Adv. Opt. Mater., 2016, 4, 1223.

54 A. Ozawa, M. Yamamoto, T. Tanabe, S. Hosokawa and T. Yoshida, J. Mater. Chem. A, 2020, 8, 7368.

55 S. Zhu, Q. Liang, Y. Xu, H. Fu and X. Xiao, Eur. J. Inorg. Chem., 2020, 773.

56 Y. P. Zhang, L. L. Wang, H. Xu, J. M. Cao, D. Chen and W. Han, Adv. Funct. Mater., 2020, 30, 1909372.

57 W. F. Li, Y. M. Yang, G. Zhang and Y. W. Zhang, Nano Lett., 2015, 15, 1691.

58 J. Mei, T. W. He, Q. Zhang, T. Liao, A. J. Du, G. A. Ayoko and Z. Q. Sun, ACS Appl. Mater. Interfaces, 2020, 12, 21720.

59 X. Y. Li, G. Chen, Z. Y. Le, X. R. Li, P. Nie, X. Y. Liu, P. C. Xu, H. B. Wu, Z. Liu and Y. F. Lu, Nano Energy, 2019, 59, 464.

60 F. C. Zhou, L. Z. Ouyang, J. W. Liu, X. S. Yang and M. Zhu, J. Power Sources, 2020, 449, 227549.

61 S. J. Zhou, J. Li, L. C. Fu, J. J. Zhu, W. L. Yang, D. Y. Li and L. P. Zhou, ChemElectroChem, 2020, 7, 2184.

62 R. Wang, X. Dai, Z. Qian, S. Zhong, S. Chen, S. Fan, H. Zhang and F. Wu, ACS Appl. Mater. Interfaces, 2020, 28, 31628 .

63 S. M. Beladi-Mousavi and M. Pumera, Chem. Soc. Rev., 2018, 47, 6964.

64 N. O. Gopal, H. H. Lo, T. F. Ke, C. H. Lee, C. C. Chou, J. D. Wu, S. C. Sheu and S. C. Ke, J. Phys. Chem. C, 2012, 116, 16191.

65 J. Jin, Y. Zheng, S. Z. Huang, P. P. Sun, N. Srikanth, L. B. Kong, Q. Y. Yan and K. Zhou, J. Mater. Chem. A, 2019, 7, 783.

66 L. Chen, G. M. Zhou, Z. B. Liu, X. M. Ma, J. Chen, Z. Y. Zhang, X. L. Ma, F. Li, H. M. Cheng and W. C. Ren, Adv. Mater., 2016, 28, 510.

67 S. Kim, J. Cui, V. P. Dravid and K. He, Adv. Mater., 2019, 31, 1904623.

68 L. Pan, X. D. Zhu, K. N. Sung, Y. T. Liu, X. M. Xie and X. Y. Ye, Nano Energy, 2016, 30, 347. 
69 J. Mei, Y. W. Zhang, T. Liao, X. M. Peng, G. A. Ayoko and Z. Q. Sun, Energy Storage Mater., 2019, 19, 424.

70 T. S. Yang, I. Abdelwahab, H. Lin, Y. Bao, S. J. R. Tan, S. Fraser, K. P. Loh and B. H. Jia, ACS Photonics, 2018, 5, 4969.

71 Q. Q. Jiang, J. Li, N. N. Yuan, Z. X. Wu and J. G. Tang, Electrochim. Acta, 2018, 263, 272.

72 L. Huang, C. Chen, X. Huang, S. Ruan and Y.-J. Zeng, Composites, Part B, 2019, 164, 583.

73 Z. Deng, C. Fang, X. Ma, X. Li, Y.-J. Zeng and X. Peng, ACS Appl. Mater. Interfaces, 2020, 12, 20321.

74 D. Zhao, L. H. Zhang, C. C. Fu, J. L. Huang, H. Y. Huang, Z. H. Li, J. Y. Zhang and C. M. Niu, Carbon, 2018, 139, 1057.

75 R. H. Wang, X. Y. Dai, Z. F. Qian, S. K. Zhong, S. Chen, S. T. Fan, H. Zhang and F. X. Wu, ACS Appl. Mater. Interfaces, 2020, 12, 31628.

76 R. Amine, A. Daali, X. W. Zhou, X. Liu, Y. Z. Liu, Y. Ren, X. Y. Zhang, L. K. Zhu, S. Al-Hallaj, Z. H. Chen, G. L. Xu and K. Amine, Nano Energy, 2020, 74, 104849.

77 K. Roy, M. Wahid, D. Puthusseri, A. Patrike, S. Muduli, R. Vaidhyanathan and S. Ogale, Sustainable Energy Fuels, 2019, 3, 245.

78 R. J. Meng, J. M. Huang, Y. T. Feng, L. H. Zu, C. X. Peng, L. R. Zheng, L. Zheng, Z. B. Chen, G. L. Liu, B. J. Chen, Y. L. Mi and J. H. Yang, Adv. Energy Mater., 2018, 8, 1801514.

79 H. C. Jin, S. Xin, C. H. Chuang, W. D. Li, H. Y. Wang, J. Zhu, H. Y. Xie, T. M. Zhang, Y. Y. Wan, Z. K. Qi, W. S. Yan, Y. R. Lu, T. S. Chan, X. J. Wu, J. B. Goodenough, H. X. Ji and X. F. Duan, Science, 2020, 370, 192.

80 S. Haghighat-Shishavan, M. Nazarian-Samani, M. Nazarian-Samani, H. K. Roh, K. Y. Chung, B. W. Cho, S. F. Kashani-Bozorg and K. B. Kim, J. Mater. Chem. A, 2018, 6, 10121.

81 H. W. Liu, L. Tao, Y. Q. Zhang, C. Xie, P. Zhou, H. B. Liu, R. Chen and S. Y. Wang, ACS Appl. Mater. Interfaces, 2017, 9, 36849.

82 Y. Zhang, W. P. Sun, Z. Z. Luo, Y. Zheng, Z. W. Yu, D. Zhang, J. Yang, H. T. Tan, J. X. Zhu, X. L. Wang, Q. Y. Yan and S. X. Dou, Nano Energy, 2017, 40, 576.

83 H. Li, A. M. Liu, X. F. Ren, Y. N. Yang, L. G. Gao, M. Q. Fan and T. L. Ma, Nanoscale, 2019, 11, 19862.

84 H. C. Jin, T. M. Zhang, C. H. Chuang, Y. R. Lu, T. S. Chan, Z. Z. Du, H. X. Ji and L. J. Wan, ACS Appl. Mater. Interfaces, 2019, 11, 16656.

85 T. B. Song, H. Chen, Z. Li, Q. J. Xu, H. M. Liu, Y. G. Wang and Y. Y. Xia, Adv. Funct. Mater., 2019, 29, 1900535.

86 X. Zhuang, K. Li and T.-Y. Zhang, Nanoscale, 2020, 12, 19609.

87 M. Y. Li, N. Muralidharan, K. Moyer and C. L. Pint, Nanoscale, 2018, 10, 10443.

88 T. B. Song, H. Chen, Q. J. Xu, H. M. Liu, Y. G. Wang and Y. Y. Xia, ACS Appl. Mater. Interfaces, 2018, 10, 37163.

89 W. W. Yang, Y. X. Lu, C. X. Zhao and H. L. Liu, Electron. Mater. Lett., 2020, 16, 89.
90 C. He, J. H. Zhang, W. X. Zhang and T. T. Li, J. Phys. Chem. $C, 2019,123,5157$.

91 I. Sultana, M. M. Rahman, T. Ramireddy, Y. Chen and A. M. Glushenkov, J. Mater. Chem. A, 2017, 5, 23506.

92 Z. L. Xu, S. H. Lin, N. Onofrio, L. M. Zhou, F. Y. Shi, W. Lu, K. Kang, Q. Zhang and S. P. Lau, Nat. Commun., 2018, 9, 4164.

93 J. Sun, Y. M. Sun, M. Pasta, G. M. Zhou, Y. Z. Li, W. Liu, F. Xiong and Y. Cui, Adv. Mater., 2016, 28, 9797.

94 Q. Zhang, Y. H. Xiao, Y. Y. Fu, C. Li, X. F. Zhang, J. Yan, J. Q. Liu and Y. C. Wu, Appl. Surf. Sci., 2020, 512, 145639.

95 L. Li, L. Chen, S. Mukherjee, J. Gao, H. Sun, Z. B. Liu, X. L. Ma, T. Gupta, C. V. Singh, W. C. Ren, H. M. Cheng and N. Koratkar, Adv. Mater., 2017, 29, 1602734.

96 H. Cheng, J. Xie, G. S. Cao, Y. H. Lu, D. Zheng, Y. Jin, K. Y. Wang and X. B. Zhao, Energy Storage Mater., 2019, 23, 684.

97 S. Yang, M. L. Bo, C. Peng, Y. D. Li and Y. Li, Mater. Lett., 2018, 233, 118.

98 W. Zhang, J. Mao, S. Li, Z. Chen and Z. Guo, J. Am. Chem. Soc., 2017, 139, 3316.

99 J. M. Sangster, J. Phase Equilibria Diffus., 2010, 31, 68.

100 Y. Kim, Y. Park, A. Choi, N. S. Choi, J. Kim, J. Lee, J. H. Ryu, S. M. Oh and K. T. Lee, Adv. Mater., 2013, 25, 3045.

101 J. Sun, G. Zheng, H.-W. Lee, N. Liu, H. Wang, H. Yao, W. Yang and Y. Cui, Nano Lett., 2014, 14, 4573.

102 Y. Yang, G. Y. Zheng and Y. Cui, Chem. Soc. Rev., 2013, 42, 3018.

103 X. L. Ji, K. T. Lee and L. F. Nazar, Nat. Mater., 2009, 8, 500. 104 Y. S. Su and A. Manthiram, Nat. Commun., 2012, 3, 1166.

105 T. Z. Hou, X. Chen, H. J. Peng, J. Q. Huang, B. Q. Li, Q. Zhang and B. Li, Small, 2016, 12, 3283.

106 P. G. Bruce, S. A. Freunberger, L. J. Hardwick and J. M. Tarascon, Nat. Mater., 2012, 11, 19.

107 N. Imanishi and O. Yamamoto, Mater. Today, 2014, 17, 24.

108 J. P. Liu, C. Guan, C. Zhou, Z. Fan, Q. Q. Ke, G. Z. Zhang, C. Liu and J. Wang, Adv. Mater., 2016, 28, 8732.

109 P. J. Lian, B. S. Zhao, L. Q. Zhang, N. Xu, M. T. Wu and X. P. Gao, J. Mater. Chem. A, 2019, 7, 20540.

110 X. J. Wu, Y. J. Xu, Y. Hu, G. Wu, H. Y. Cheng, Q. Yu, K. Zhang, W. Chen and S. Chen, Nat. Commun., 2018, 9, 4573.

111 A. Sajedi-Moghaddam, C. C. Mayorga-Martinez, Z. Sofer, D. Bousa, E. Saievar-Iranizad and M. Pumera, J. Phys. Chem. C, 2017, 121, 20532.

112 X. Y. Fu, Z. D. Chen, Y. L. Zhang, D. D. Han, J. N. Ma, W. Wang, Z. R. Zhang, H. Xia and H. B. Sun, Nanoscale, 2019, 11, 9133.

113 M. Wen, D. N. Liu, Y. H. Kang, J. H. Wang, H. Huang, J. Li, P. K. Chu and X. F. Yu, Mater. Horiz., 2019, 6, 176.

114 S. J. Luo, J. L. Zhao, J. F. Zou, Z. L. He, C. W. Xu, F. W. Liu, Y. Huang, L. Dong, L. Wang and H. Zhang, ACS Appl. Mater. Interfaces, 2018, 10, 3538.

115 C. X. Hao, B. C. Yang, F. S. Wen, J. Y. Xiang, L. Li, W. H. Wang, Z. M. Zeng, B. Xu, Z. S. Zhao, Z. Y. Liu and Y. J. Tian, Adv. Mater., 2016, 28, 3194. 
116 B. C. Yang, C. X. Hao, F. S. Wen, B. C. Wang, C. P. Mu, J. Y. Xiang, L. Li, B. Xu, Z. S. Zhao, Z. Y. Liu and Y. J. Tian, ACS Appl. Mater. Interfaces, 2017, 9, 44478.

117 X. H. Chen, G. G. Xu, X. H. Ren, Z. J. Li, X. Qi, K. Huang, H. Zhang, Z. Y. Huang and J. X. Zhong, J. Mater. Chem. A, 2017, 5, 6581.

118 G. Wu, P. F. Tan, X. J. Wu, L. Peng, H. Y. Cheng, C. F. Wang, W. Chen, Z. Y. Yu and S. Chen, Adv. Funct. Mater., 2017, 27, 1702493.

119 J. Y. Cao, P. He, J. R. Brent, H. Yilmaz, D. J. Lewis, I. A. Kinloch and B. Derbye, ACS Appl. Mater. Interfaces, 2018, 10, 10330.

120 Z. S. Cao, Z. C. Yang, C. Zhang, L. Q. Xie, X. H. Zhao, P. Pan, Y. J. Yuan, W. Qi, J. He, H. H. Zhang, T. Xue, P. Zhang, J. Wei, K. L. Zhang and J. S. Zhao, J. Mater. Sci.: Mater. Electron., 2020, 31, 2406.

121 A. Gopalakrishnan and S. Badhulika, Chem. Commun., 2020, 56, 7096.

122 L. J. Zhao, Z. Sun, H. X. Wan, H. Liu, D. Z. Wu, X. D. Wang and X. G. Cui, Electrochim. Acta, 2020, 354, 136718.

123 L. Q. Kong, Y. J. Ji, Z. Z. Dang, J. Q. Yan, P. Li, Y. Y. Li and S. Z. Liu, Adv. Funct. Mater., 2018, 28, 1800668.

124 Y. J. Yuan, P. Wang, Z. J. Li, Y. Z. Wu, W. F. Bai, Y. B. Su, J. Guan, S. T. Wu, J. S. Zhong, Z. T. Yu and Z. G. Zou, Appl. Catal., B, 2019, 242, 1.

125 Y. J. Yuan, Z. K. Shen, S. X. Song, J. Guan, L. Bao, L. Pei, Y. B. Su, S. T. Wu, W. F. Bai, Z. T. Yu, Z. G. Ji and Z. G. Zou, ACS Catal., 2019, 9, 7801.

126 X. Y. Cai, L. Mao, S. Q. Yang, K. L. Han and J. Y. Zhang, ACS Energy Lett., 2018, 3, 932.

127 T. Song, G. C. Zeng, P. Y. Zhang, T. T. Wang, A. Ali, S. B. Huang and H. P. Zeng, J. Mater. Chem. A, 2019, 7, 503.

128 M. S. Zhu, M. Fujitsuka, L. X. Zeng, M. H. Liu and T. Majima, Appl. Catal., B, 2019, 256, 117864.

129 J. D. Hu, D. Y. Chen, Z. Mo, N. J. Li, Q. F. Xu, H. Li, J. H. He, H. Xu and J. M. Lu, Angew. Chem., Int. Ed., 2019, 58, 2073.

130 X. Wang, J. He, J. Li, G. Lu, F. Dong, T. Majima and M. Zhu, Appl. Catal., B, 2020, 277, 119230.

131 X. W. Zhu, S. Q. Huang, Q. Yu, Y. B. She, J. M. Yang, G. L. Zhou, Q. D. Li, X. J. She, J. J. Deng, H. M. Li and H. Xu, Appl. Catal., B, 2020, 269, 118760.

132 J. Z. Huang, X. R. Guo, J. Yang and L. S. Wang, J. CO2 Util., 2020, 38, 32 .

133 W. Lei, T. Zhang, P. Liu, J. A. Rodriguez, G. Liu and M. Liu, ACS Catal., 2016, 6, 8009.

134 X. Zhu, T. Zhang, Z. Sun, H. Chen, J. Guan, X. Chen, H. Ji, P. Du and S. Yang, Adv. Mater., 2017, 29, 1605776.

135 R. Marschall, Adv. Funct. Mater., 2014, 24, 2421.

136 J. H. Wu, S. L. Huang, Z. Y. Jin, J. Q. Chen, L. Hu, Y. J. Long, J. G. Lu, S. C. Ruan and Y. J. Zeng, J. Mater. Sci., 2018, 53, 16557.

137 C. Hepburn, E. Adlen, J. Beddington, E. A. Carter, S. Fuss, N. Mac Dowell, J. C. Minx, P. Smith and C. K. Williams, Nature, 2019, 575, 87.

138 X. Li, J. G. Yu, M. Jaroniec and X. B. Chen, Chem. Rev., 2019, 119, 3962.
139 Z. K. Shen, Y. J. Yuan, P. Wang, W. F. Bai, L. Pei, S. T. Wu, Z. T. Yu and Z. G. Zou, ACS Appl. Mater. Interfaces, 2020, 12, 17343.

140 P. X. Qiu, C. M. Xu, N. Zhou, H. Chen and F. Jiang, Appl. Catal., B, 2018, 221, 27.

141 S. Bian, M. Wen, J. H. Wang, N. Yang, P. K. Chu and X. F. Yu, J. Phys. Chem. Lett., 2020, 11, 1052.

142 M. Vesely, P. Marvan, J. Trejbal, V. Mazanek, J. Luxa, J. Sturala and Z. Sofer, ACS Appl. Mater. Interfaces, 2020, 12, 22702.

143 W. J. Wang, Q. Y. Niu, G. M. Zeng, C. Zhang, D. L. Huang, B. B. Shao, C. Y. Zhou, Y. Yang, Y. X. Liu, H. Guo, W. P. Xiong, L. Lei, S. Y. Liu, H. Yi, S. Chen and X. Tang, Appl. Catal., B, 2020, 273, 119051.

144 Y. Zheng, Z. H. Yu, H. H. Ou, A. M. Asiri, Y. L. Chen and X. C. Wang, Adv. Funct. Mater., 2018, 28, 1705407.

145 J. Q. Yan, Y. J. Ji, L. Q. Kong, Y. Y. Li, M. Navlani-Garcia, S. Z. Liu, Y. Kuwahara, K. Mori, M. Che and H. Yamashita, ChemCatChem, 2018, 10, 3424.

146 N. Zhang, A. Jalil, D. X. Wu, S. M. Chen, Y. F. Liu, C. Gao, W. Ye, Z. M. Qi, H. X. Ju, C. M. Wang, X. J. Wu, L. Song, J. F. Zhu and Y. J. Xiong, J. Am. Chem. Soc., 2018, 140, 9434.

147 C. C. Mayorga-Martinez, N. M. Latiff, A. Y. S. Eng, Z. Sofer and M. Pumera, Anal. Chem., 2016, 88, 10074.

148 Y. Lin, Y. Pan and J. Zhang, Int. J. Hydrogen Energy, 2017, 42, 7951.

149 R. He, J. Hua, A. Q. Zhang, C. H. Wang, J. Y. Peng, W. J. Chen and J. Zeng, Nano Lett., 2017, 17, 4311.

150 L. Y. Shao, H. M. Sun, L. C. Miao, X. Chen, M. Han, J. C. Sun, S. Liu, L. Li, F. Y. Cheng and J. Chen, J. Mater. Chem. A, 2018, 6, 2494.

151 Q. Q. Jiang, L. Xu, N. Chen, H. Zhang, L. M. Dai and S. Y. Wang, Angew. Chem., Int. Ed., 2016, 55, 13849.

152 Z. M. Zhang, M. Khurram, Z. J. Sun and Q. F. Yan, Inorg. Chem., 2018, 57, 4098.

153 T. Wu, J. C. Fan, Q. X. Li, P. H. Shi, Q. J. Xu and Y. L. Min, Adv. Energy Mater., 2018, 8, 1701799.

154 D. N. Liu, J. H. Wang, S. Bian, Q. Liu, Y. H. Gao, X. Wang, P. K. Chu and X. F. Yu, Adv. Funct. Mater., 2020, 30, 2002731.

155 M. Batmunkh, A. Shrestha, M. Bat-Erdene, M. J. Nine, C. J. Shearer, C. T. Gibson, A. D. Slattery, S. A. Tawfik, M. J. Ford, S. Dai, S. Z. Qiao and J. G. Shapter, Angew. Chem., Int. Ed., 2018, 57, 2644.

156 S. L. Jiao, X. W. Fu, L. Zhang, Y. J. Zeng and H. W. Huang, Nano Today, 2020, 31, 100833.

157 K. P. Gong, F. Du, Z. H. Xia, M. Durstock and L. M. Dai, Science, 2009, 323, 760.

158 J. H. Wang, D. N. Liu, H. Huang, N. Yang, B. Yu, M. Wen, X. Wang, P. K. Chu and X. F. Yu, Angew. Chem., Int. Ed., 2018, 57, 2600.

159 M. M. Liu, R. Z. Zhang and W. Chen, Chem. Rev., 2014, 114, 5117.

160 C. X. Guo, J. R. Ran, A. Vasileff and S. Z. Qiao, Energy Environ. Sci., 2018, 11, 45.

161 Y. X. Lin, S. N. Zhang, Z. H. Xue, J. J. Zhang, H. Su, T. J. Zhao, G. Y. Zhai, X. H. Li, M. Antonietti and J. S. Chen, Nat. Commun., 2019, 10, 4380. 
162 C. Wang, J. Gao, J. G. Zhao, D. J. Yan and X. D. Zhu, Small, 2020, 16, 1907091.

163 Y. T. Liu, D. Li, J. Y. Yu and B. Ding, Angew. Chem., Int. Ed., 2019, 58, 16439.

164 J. D. Wood, S. A. Wells, D. Jariwala, K. S. Chen, E. Cho, V. K. Sangwan, X. L. Liu, L. J. Lauhon, T. J. Marks and M. C. Hersam, Nano Lett., 2014, 14, 6964.

165 Y. T. Zhao, H. Y. Wang, H. Huang, Q. L. Xiao, Y. H. Xu, Z. N. Guo, H. H. Xie, J. D. Shao, Z. B. Sun, W. J. Han, X. F. Yu, P. H. Li and P. K. Chu, Angew. Chem., Int. Ed., 2016, 55, 5003.

166 M. T. Edmonds, A. Tadich, A. Carvalho, A. Ziletti, K. M. O’Donnell, S. P. Koenig, D. F. Coker, B. Ozyilmaz, A. H. C. Neto and M. S. Fuhrer, ACS Appl. Mater. Interfaces, 2015, 7, 14557.

167 Y. H. Zhao, Q. H. Zhou, Q. Li, X. J. Yao and J. L. Wang, Adv. Mater., 2017, 29, 1603990.
168 C. R. Ryder, J. D. Wood, S. A. Wells, Y. Yang, D. Jariwala, T. J. Marks, G. C. Schatz and M. C. Hersam, Nat. Chem., 2016, 8, 597.

169 X. K. Huang, D. Liu, X. R. Guo, X. Y. Sui, D. Y. Qu and J. H. Chen, ACS Sustainable Chem. Eng., 2018, 6, 16308.

170 J. Y. Cao, P. He, J. R. Brent, H. Yilmaz, D. J. Lewis, I. A. Kinloch and B. Derby, ACS Appl. Mater. Interfaces, 2018, 10, 10330.

171 J. H. Wang, D. N. Liu, H. Huang, N. Yang, B. Yu, M. Wen, X. Wang, P. K. Chu and X. F. Yu, Angew. Chem., Int. Ed., 2018, 57, 2600.

172 Y. Liu, B. N. Shivananju, Y. S. Wang, Y. P. Zhang, W. Z. Yu, S. Xiao, T. Sun, W. L. Ma, H. R. Mu, S. H. Lin, H. Zhang, Y. R. Lu, C. W. Qiu, S. J. Li and Q. L. Bao, ACS Appl. Mater. Interfaces, 2017, 9, 36137.

173 S. Kuriakose, T. Ahmed, P. Taylor, Y. Zhu, M. Spencer, S. Balendhran, Y. R. Lu, V. Bansal, S. Sriram, M. Bhaskaran and S. Walia, 2D Mater., 2018, 6, 015009. 